Check for updates

Cite this: Chem. Soc. Rev., 2020, 49, 3952

Received 19th February 2020

DOI: $10.1039 / \mathrm{d} 0 \operatorname{cs} 00143 k$

rsc.li/chem-soc-rev

\section{Direct synthesis of metastable phases of 2D transition metal dichalcogenides}

\author{
Maria S. Sokolikova (D) and Cecilia Mattevi (D)*
}

The different polymorphic phases of transition metal dichalcogenides (TMDs) have attracted enormous interest in the last decade. The metastable metallic and small band gap phases of group VI TMDs displayed leading performance for electrocatalytic hydrogen evolution, high volumetric capacitance and some of them exhibit large gap quantum spin Hall (QSH) insulating behaviour. Metastable $1 T\left(1 T^{\prime}\right)$ phases require higher formation energy, as compared to the thermodynamically stable $2 \mathrm{H}$ phase, thus in standard chemical vapour deposition and vapour transport processes the materials normally grow in the $2 \mathrm{H}$ phases. Only destabilization of their $2 \mathrm{H}$ phase via external means, such as charge transfer or high electric field, allows the conversion of the crystal structure into the $1 \mathrm{~T}\left(1 \mathrm{~T}^{\prime}\right)$ phase. Bottom-up synthesis of materials in the $1 \mathrm{~T}\left(1 \mathrm{~T}^{\prime}\right)$ phases in measurable quantities would broaden their prospective applications and practical utilization. There is an emerging evidence that some of these $1 \mathrm{~T}\left(1 \mathrm{~T}^{\prime}\right)$ phases can be directly synthesized via bottom-up vapour- and liquid-phase methods. This review will provide an overview of the synthesis strategies which have been designed to achieve the crystal phase control in TMDs, and the chemical mechanisms that can drive the synthesis of metastable phases. We will provide a critical comparison between growth pathways in vapour- and liquid-phase synthesis techniques. Morphological and chemical characteristics of synthesized materials will be described along with their ability to act as electrocatalysts for the hydrogen evolution reaction from water. Phase stability and reversibility will be discussed and new potential applications will be introduced. This review aims at providing insights into the fundamental understanding of the favourable synthetic conditions for the stabilization of metastable TMD crystals and at stimulating future advancements in the field of large-scale synthesis of materials with crystal phase control.

Department of Materials, Imperial College London, London SW7 2AZ, UK. E-mail: c.mattevi@imperial.ac.uk

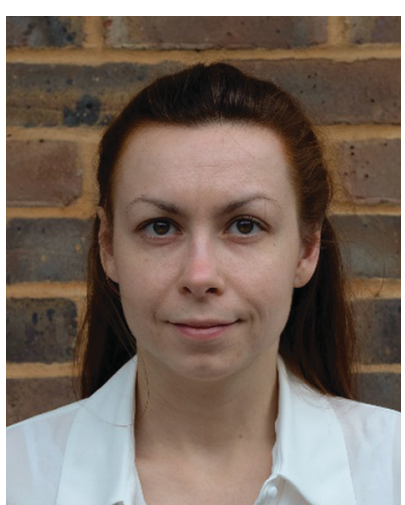

Maria S. Sokolikova
Maria Sokolikova received her MSc degree in Chemistry from Lomonosov Moscow State University in 2013. She then joined Dr Mattevi research group at Imperial College London as a PhD student holding the President's Scholarship. Maria completed her PhD in Materials Science from Imperial College London in 2020. Presently, Maria is a postdoctoral researcher in the Department of Materials at Imperial College London and her research focuses on colloidal growth of $2 D$ materials for electrocatalysis.

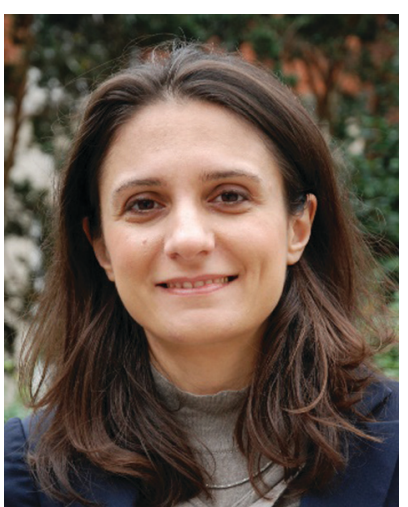

Cecilia Mattevi
Dr Cecilia Mattevi received her Laurea degree in Materials Science from the University of Padua and a PhD in Materials Science from the University of Padua in 2008. After a postdoctoral appointment at Rutgers University, $\mathrm{Dr}$ Mattevi joined the Materials Department at Imperial College London, becoming a Junior Research Fellow in 2010 and then a Lecturer and Royal Society University Research Fellow in

2012. Cecilia is a Fellow of the Royal Society of Chemistry and her research interest includes the liquid phase synthesis of $2 D$ materials with tailored characteristics and the investigation of their properties for energy applications. 


\section{Introduction}

The isolation of graphene has sparked widespread interest in layered materials. A large family of those materials is represented by the transition metal dichalcogenides which comprise about 40 compounds with a chemical formula of $\mathrm{ME}_{2}$ (M, transition metal; E, chalcogen). ${ }^{1}$ While they have been studied for over half a century in their bulk form ${ }^{2,3}$ for their photoelectrocatalytic activity, ${ }^{4-6}$ lubricity $^{7}$ to superconductivity, ${ }^{8,9}$ it was not until recently that they have been isolated in their elementary building blocks, which are triatomic layers, and several of their properties started to emerge and their potential identified. These emerging 2D materials offer new opportunities for the realization of novel technologies and for the exploration of novel condensed matter phenomena.

Interestingly, compounds of group IV, V, VI, VII and X are nearly isostructural with three possible crystallographic orders in the monolayer form, ${ }^{10}$ and they present several analogies. Group VI TMDs have been studied more extensively than others and in particular the Se and $\mathrm{S}$ compounds $\left(\mathrm{MoS}_{2}, \mathrm{MoSe}_{2}, \mathrm{WS}_{2}\right.$ and $\mathrm{WSe}_{2}$ ) owing to the richness and the uniqueness of the properties concerning each of their polymorphs. Additionally, the relative abundance of transition metal compounds, the good chemical stability in air compared to the dichalcogenides of other groups metals, make them more accessible. Group VI TMDs started to attract large interest a decade ago owing to the ability to display an indirect-to-direct bandgap crossover with bright photoluminescence (PL) in the monolayer form at room temperature $^{11}$ and for the valley degree of freedom associated to the non-centrosymmetric semiconducting phase. ${ }^{12}$ Additional unique properties to this class of materials include mechanical flexibility, electrostatic coupling, large carrier mobility, gatetunability, piezoelectricity in monolayers and odd number of layers, as discussed in recent reviews. ${ }^{13-16}$

It is now emerging that there is a variety of other properties associated to different polymorphs of group VI TMDs; some of those arising from the different crystallographic phases that they can acquire. In addition to the thermodynamically stable $2 \mathrm{H}$ polymorph with the trigonal-prismatic coordination of transition metal atoms, there are various polymorphs that are broadly defined as $1 \mathrm{~T}$ phases (perfect $1 \mathrm{~T}$ and distorted $1 \mathrm{~T}^{\prime}$, $1 \mathrm{~T}^{\prime \prime}$ and $1 \mathrm{~T}^{\prime \prime \prime}$ ) and where the metal atoms present an octahedral (or distorted octahedral) coordination. These phases are generally characterized by a metal- $(1 \mathrm{~T})$ or semimetal-like $\left(1 \mathrm{~T}^{\prime}\right)$ behaviour.

Indeed, the electronic band structure is heavily affected by the coordination of transition metal atoms which leads to a different splitting of the d-orbitals. The key opportunity arising from the TMD polymorphism is to influence the electronic structure of a given material and so to achieve a high density of electronic states at the Fermi level, which can enable efficient electrocatalytic hydrogen evolution from water. This ability was initially attributed to the localized metallic states on molybdenum-terminated edges of $2 \mathrm{H} \mathrm{MoS}$ clusters. ${ }^{17}$ Soon after, it became clear that the basal planes of the $1 \mathrm{~T}$ phase $\mathrm{MoS}_{2}$ or $\mathrm{WS}_{2}$ could enable accessibility to a large number of electrocatalytically active sites thus approaching Pt performance. ${ }^{18}$
Several $1 \mathrm{~T}^{\prime}$ polymorphs have been demonstrated since. The $1 \mathrm{~T}^{\prime}$ phases of single layer $\mathrm{WSe}_{2}$ and $\mathrm{WTe}_{2}$ have been reported to be large gap quantum spin Hall (QSH) insulators suitable for application in spintronic devices and in the case of $\mathrm{WSe}_{2}$ also operable at ambient temperature. ${ }^{19-21}$ Their chemical stability makes them more advantageous of the currently known large gap QSH insulators such as stanene ${ }^{22}$ and two-dimensional In-Sb compounds which can be used in an inert atmosphere only. ${ }^{23}$ On the other hand, it is predicted that a spontaneous symmetry breaking in the undistorted metallic $1 \mathrm{~T}$ phase would open up a band gap and lead to the emergence of robust ferroelectricity in the novel $1 \mathrm{~T}^{\prime \prime \prime}$ phase. ${ }^{24}$

Despite the rapid progress in investigating the properties of $1 \mathrm{~T}\left(1 \mathrm{~T}^{\prime}\right)$ phases of group VI TMDs over a small scale (about hundreds of nanometres over the basal plane), it is still quite arduous to obtain those phases as continuous over large areas. The $1 \mathrm{~T}\left(1 \mathrm{~T}^{\prime}\right)$ phases are metastable, thus their direct synthesis is very challenging because the formation energy of the $1 \mathrm{~T}^{\prime}$ phase is higher than that of the $2 \mathrm{H}$ phase $;^{25}$ therefore, the $2 \mathrm{H} \mathrm{ME} 2$ is the preferable phase obtained.

The most common way, and historically the first one to be used, for achieving those $1 \mathrm{~T}\left(1 \mathrm{~T}^{\prime}\right)$ phases is via conversion of the $2 \mathrm{H}$ phase. This is generally achieved by increasing the electron density via exposure to alkali metals. ${ }^{26,27}$ While the design of direct synthesis methods for the $1 \mathrm{~T}\left(1 \mathrm{~T}^{\prime}\right)$ phases is still at the early stages of investigation. Here, we provide a comprehensive overview of topochemical methods and direct synthesis methods designed so far to obtain the $1 \mathrm{~T}\left(1 \mathrm{~T}^{\prime}\right)$ phases of group VI TMDs. We will discuss the chemical prerequisites for the formation of the $1 \mathrm{~T}\left(1 \mathrm{~T}^{\prime}\right)$ phases in vapour phase and liquid phase. We will present the possible reaction pathways that can take place in liquid phase which lead to the nucleation and growth of crystalline metastable phases.

We will then review the intrinsic properties of $1 \mathrm{~T}\left(1 \mathrm{~T}^{\prime}\right)$ phases and we highlight the differences in the materials morphology and properties between $1 \mathrm{~T}\left(1 \mathrm{~T}^{\prime}\right)$ phases obtained via destabilization of $2 \mathrm{H}$ phase versus direct synthetic approaches. Finally, we will introduce newly emerging properties which are unique to the $1 \mathrm{~T}\left(1 \mathrm{~T}^{\prime}\right)$ phases as they arise from the lower symmetry. We will conclude the review with an outlook for the future of the different synthetic approaches to metastable phases in view of their applications and possible large-scale production of these materials.

\section{Structural polymorphs of group VI TMDs}

TMDs exhibit a variety of structural polymorphs. The polymorphs are determined by the very different chalcogen coordination geometry that transition metal atoms can accommodate, while the polytypes are determined by the stacking order of two or more individual triatomic layers with the same symmetry.

1.1. 1H-type phases of group VI TMDs. The first polymorph of group VI TMDs to be considered is characterised by a trigonal-prismatic coordination of transition metal atoms (point group $D_{3 \mathrm{~h}}$ ). In a single layer form, it is denoted as the $1 \mathrm{H}$ phase and its crystallographic projections along both the $b$ (side view) and $c$ (top view) axes are shown in Fig. 1. Depending on 


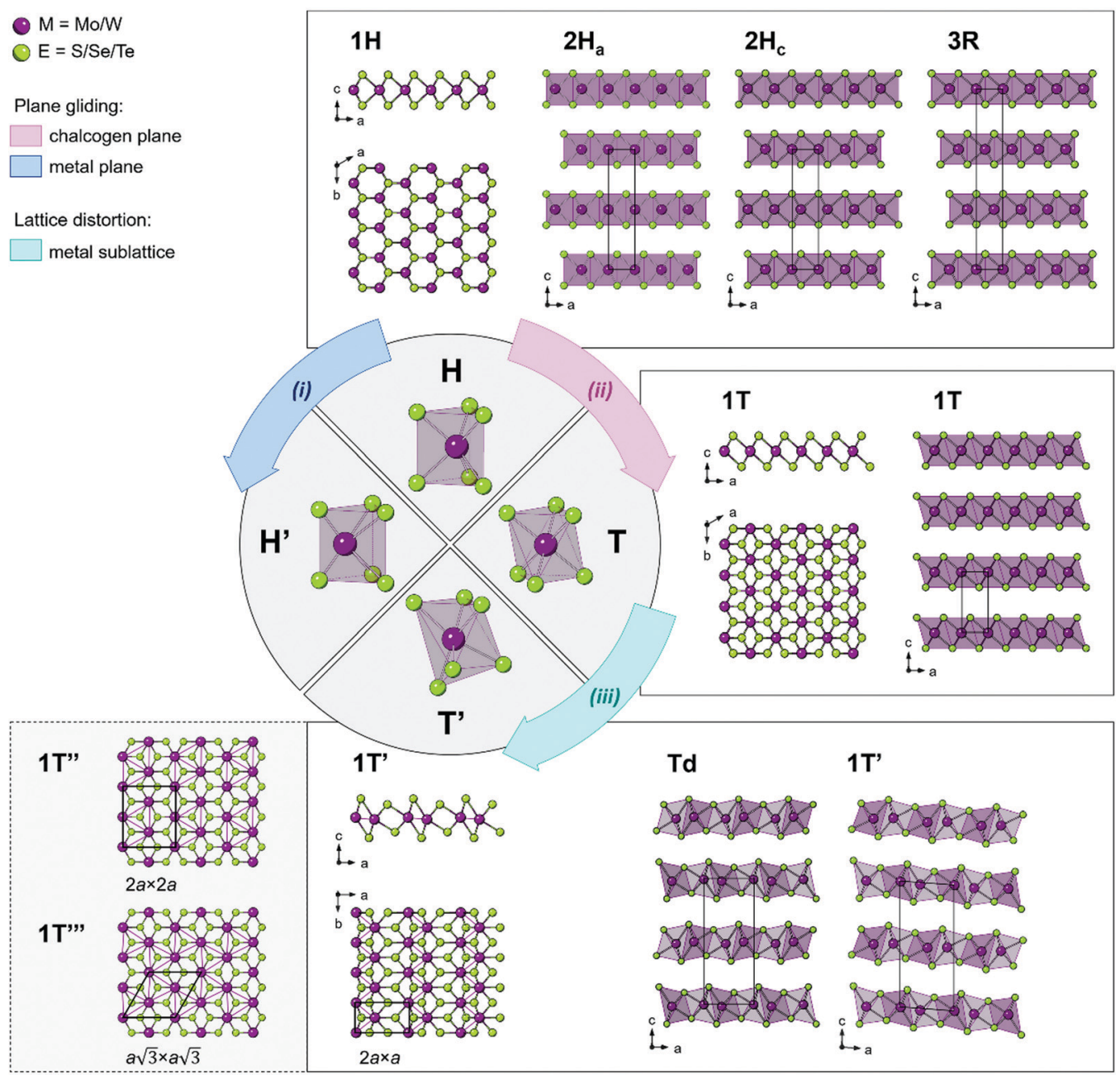

Fig. 1 Bulk polymorphs of group VI TMDs. In the schematics, the arrows indicate the pathways for possible structural transformation (i) from the $1 \mathrm{H}$ to the $1 \mathrm{H}^{\prime}$ phase, (ii) from the $1 \mathrm{H}$ to the $1 \mathrm{~T}$ phase, and (iii) from the $1 \mathrm{~T}$ to the $1 \mathrm{~T}^{\prime}$ phase via either (i) metal plane gliding, (ii) chalcogen plane gliding, or (iii) spontaneous distortion in the metal sublattice. The crystallographic projections of the $1 \mathrm{H}, 1 \mathrm{~T}$, and $1 \mathrm{~T}^{\prime}$ monolayers along the $b$ (side view) and $c$ (top view) axes are shown. The known polytypes (stacking polymorphs) of the $1 \mathrm{H}, 1 \mathrm{~T}$, and $1 \mathrm{~T}^{\prime}$ structures are presented in the corresponding fields. Bottom left panel illustrates two additional clustering patterns - tetramerization (1T') and trimerization $\left(1 T^{\prime \prime \prime}\right)$ - proposed in the distorted $1 T^{\prime} \mathrm{TMDs}$.

the stacking sequence of individual $1 \mathrm{H}$ triatomic layers, the $2 \mathrm{H}_{\mathrm{a}}, 2 \mathrm{H}_{\mathrm{c}}$ and $3 \mathrm{R}$ polytypes are distinguished, where $\mathrm{H}$ or $\mathrm{R}$ stands for the hexagonal or rhombohedral lattice system and the integer denotes the number of triatomic layers in a unit cell. The $A b A B a B$ and $A c A$ $B c B$ stacking sequences result in the $2 \mathrm{H}_{\mathrm{a}}$ and $2 \mathrm{H}_{\mathrm{c}}$ polytypes, respectively, while the $3 \mathrm{R}$ phase displays the $A b A B c B C a C$ sequence of staggered $1 \mathrm{H}$ layers sharing the same crystallographic orientation. $^{1,28}$ These structural polytypes are presented in Fig. 1. Under ambient conditions, the thermodynamically stable polymorph of the majority of group VI TMDs is the semiconducting $2 \mathrm{H}_{\mathrm{c}}$ phase, ${ }^{25,29-31}$ whereas the $2 \mathrm{H}_{\mathrm{a}}$ phase is common in group V TMDs, such as $\mathrm{NbSe}_{2}$, and is metastable for group VI TMDs. ${ }^{32,33}$ It has been predicted that the $2 \mathrm{H}_{\mathrm{c}}-\mathrm{to}-2 \mathrm{H}_{\mathrm{a}}$ phase transition can be induced in bulk $\mathrm{MoS}_{2}$ under pressure (13 GPa); however, such a transition is not expected in $\mathrm{MoSe}_{2}$ and $\mathrm{MoTe}_{2}$ since in these two cases the energy barrier of the structural transformation increases with pressure. ${ }^{33}$ The experiments have indeed demonstrated that the $2 \mathrm{H}_{\mathrm{c}}-\mathrm{to}_{\mathrm{O}}-2 \mathrm{H}_{\mathrm{a}}$ phase transition in $\mathrm{MoS}_{2}$ occurs under applied pressure of 19-40
GPa and is accompanied by a collapse in the $c$ parameter leading to an overlap of the valence and conduction bands and to a metallic character of the $2 \mathrm{H}_{\mathrm{a}}$ phase. ${ }^{34,35}$ In the $2 \mathrm{H}$ structure, the second layer is rotated by $60^{\circ}$ around the $c$ axis with respect to the first layer, whereas in the 3R structure all layers have the same orientation but are shifted with respect to one another, and this might be the reason why the $2 \mathrm{H}$-to-3R transitions were not reported so far. Similar to the $1 \mathrm{H}$ single layers of group VI TMDs demonstrating a robust spinvalley coupled physics, ${ }^{12,36-38}$ the semiconducting $3 \mathrm{R}$ polymorph lacks the inversion symmetry and therefore provides an ideal platform for realisation of spin- and valleytronic devices as well as of ultrathin nonlinear optical devices. ${ }^{39}$ Direct synthesis of the phase-pure $3 \mathrm{R}$ polymorph is challenging and has only been reported via chemical vapour transport (CVT) approach. ${ }^{39-41}$

Two types of structural transitions in the $1 \mathrm{H}$-type TMD monolayers associated with the atomic planes gliding within one triatomic layer are assumed to give rise to two distinct polymorphs: $1 \mathrm{H}^{\prime}$ and $1 \mathrm{~T}^{42}$ (Fig. 1, paths i and ii). The transversal 
displacement of the transition metal plane could produce the distorted $2 \mathrm{H}$ phase, which is referred to as the $2 \mathrm{H}^{\prime}$ phase and is considered as a $60^{\circ}$ rotational phase of the $2 \mathrm{H}$ structure ${ }^{42}$ (Fig. 1, path i). Experimentally, the formation of a new distorted $2 \mathrm{H}$ phase, $2 \mathrm{H}_{\mathrm{d}}$, was observed in the few-layered $2 \mathrm{H} \mathrm{MoTe} \mathrm{M}_{2}$ and $\mathrm{W}_{x} \mathrm{Mo}_{1-x} \mathrm{Te}_{2}$ when an external electric field was applied. ${ }^{43}$ The phase was found to be unstable and is considered to be a transitional structural state between the semiconducting $2 \mathrm{H}_{\mathrm{c}}$ and the semimetallic $1 \mathrm{~T}^{\prime}$ (or $\mathrm{Td}$ ) phases discussed below. The structure of the mentioned $2 \mathrm{H}_{\mathrm{d}}$ state was not inferred at this stage.

1.2. 1T-type phases of group VI TMDs. On the other hand, intralayer gliding of one of the chalcogen planes, as shown in Fig. 1, path ii, produces the $1 \mathrm{~T}$ structure which can be visualised as a $1 \mathrm{H}$ three-atom thick E-M-E slab distorted along the [210] crystallographic direction. As a result of this, the metal centres adopt a perfect octahedral coordination (point group $O_{\mathrm{h}}$ ) shown in Fig. 1. In contrast to the rich polytypism of the $\mathrm{H}$ phases, only one trigonal $1 \mathrm{~T}$ polymorph (space group $P \overline{3} m 1$ ), with the metal centres of two adjacent layers located above each other, is reported in literature. ${ }^{44}$

The perfect octahedral $1 \mathrm{~T}$ structure of group VI disulphides and diselenides is typically higher in energy by 700-900 $\mathrm{meV}$ per formula unit than the trigonal-prismatic $1 \mathrm{H}$ structure, ${ }^{45}$ which renders this phase metastable. ${ }^{25,46}$ It is prone to spontaneous structural distortions, caused by charge density waves (CDW) which lead to clustering of transition metal atoms and buckling of chalcogen planes to accommodate these metal atom displacements ${ }^{47,48}$ (Fig. 1, path iii). Different distorted $1 \mathrm{~T}$ structures of chemically exfoliated $\mathrm{MoS}_{2}$ and $\mathrm{WS}_{2}$ have been proposed in literature back in 1990s. ${ }^{47}$ The most common superstructures include the zigzag chain $(2 a \times a)$ formation, tetramerization $(2 a \times 2 a)$, and trimerization $(a \sqrt{3} \times a \sqrt{3})$ of transition metal atoms in the $a b$ plane as shown in Fig. 1. The former one is widely referred to as the $1 \mathrm{~T}^{\prime}$ polymorph and was established by scanning tunnelling microscopy (STM) studies of chemically exfoliated $\mathrm{MoS}_{2}$ and $\mathrm{WS}_{2}$ single layers ${ }^{47,49-51}$ (Fig. 1). The three-dimensional arrangement of the $1 \mathrm{~T}^{\prime}$ single layers can lead to the formation of two polytypes: the orthorhombic $\mathrm{Td}$ phase and the monoclinic $1 \mathrm{~T}^{\prime}$ phase (Fig. 1). Additionally, tetramerization and trimerization within the transition metal sublattice would result in the $1 \mathrm{~T}^{\prime \prime}$ and $1 \mathrm{~T}^{\prime \prime \prime}$ phases, respectively. ${ }^{52}$ According to first-principles calculations, the semimetallic $1 \mathrm{~T}^{\prime}$ phase of group VI TMDs monolayers generally possesses the lowest formation energy among the competing distorted octahedral phases. ${ }^{45,53}$ However, a spontaneous trimerization of transition metal atoms, involving only a slight displacement from the equilibrium positions, is found to be favourable in $1 \mathrm{~T} \mathrm{MoS}_{2}$ monolayers, leading to the formation of the semiconducting $1 \mathrm{~T}^{\prime \prime \prime}$ phase. ${ }^{45,54}$ Such distinct distortion patterns in 1T group VI TMD monolayers are in good agreement with the early predictions that weak distortions (trimerization) are to be expected in the systems with short M-E bonds; whereas, strong distortions (zigzag chain formation) are characteristic to the systems with long $\mathrm{M}-\mathrm{E}$ bonds. ${ }^{55}$ It has also been predicted that the spontaneous symmetry breaking in
1T group VI TMD monolayers would lead to the emergence of robust ferroelectricity in the $1 \mathrm{~T}^{\prime \prime \prime}$ phase, with the electrical dipoles perpendicular to the $a b$ plane. ${ }^{24,54}$ Recently, the preparation of $1 \mathrm{~T}^{\prime \prime \prime} \mathrm{MoS}_{2}$ single crystals (space group $P 3_{1} m$ ) by deintercalation of $\mathrm{KMoS}_{2}$ crystals has been reported. ${ }^{56}$ Unlike the metallic $1 \mathrm{~T}$ and semimetallic $1 \mathrm{~T}^{\prime}$ phases, bulk $1 \mathrm{~T}^{\prime \prime \prime} \mathrm{MoS}_{2}$ was reported to be a semiconductor with an indirect band gap of $0.65 \mathrm{eV}$. Although the $1 \mathrm{~T}^{\prime \prime \prime}$ phase was predicted to be formed only in $\mathrm{MoS}_{2}$ monolayers, the small energy difference between the $1 \mathrm{~T}^{\prime}$ and $1 \mathrm{~T}^{\prime \prime \prime}$ monolayers of $\mathrm{WS}_{2}$ and $\mathrm{MoSe}_{2}$ (36.5 and $57.8 \mathrm{meV}$ per formula unit, respectively) suggests that these metastable phases might be obtained under favourable synthesis conditions. ${ }^{54}$

It is important to note that for group VI disulphides and diselenides, the $1 \mathrm{~T}^{\prime}$ polymorph is metastable under ambient conditions and can only be obtained under specific experimental conditions. On the contrary, bulk $\mathrm{WTe}_{2}$ forms the orthorhombic Td polymorph (space group Pnm2 $\left.2_{1}\right){ }^{57}$ Bulk $\mathrm{MoTe}_{2}$ is normally obtained in the $2 \mathrm{H}$ polymorph ( $\alpha$-phase); however, the monoclinic $1 \mathrm{~T}^{\prime} \mathrm{MoTe}_{2}$ polymorph (space group $P 2_{1} / m$ ) exists at temperatures above $900{ }^{\circ} \mathrm{C}$ ( $\beta$-phase). ${ }^{57,58}$ Due to a very small energy difference between the $2 \mathrm{H}$ and $1 \mathrm{~T}^{\prime}$ polymorphs of $\mathrm{MoTe}_{2}$ (40 meV per formula unit ${ }^{25}$ ), both polymorphs are reported almost equally probable depending on the synthesis conditions. ${ }^{59,60}$ The orthorhombic Td polymorph ( $\gamma$-phase) of $\mathrm{MoTe}_{2}$ can be obtained by cooling the monoclinic $1 \mathrm{~T}^{\prime}(\beta-)$ phase down to cryogenic temperatures ${ }^{61-64}$ or it can be stabilized at room temperature in the presence of substitutional doping with tungsten. ${ }^{65}$ On the other hand, the $\mathrm{Td}$ polymorph of $\mathrm{WTe}_{2}$ can be transformed into the lower symmetry $1 \mathrm{~T}^{\prime}$ phase at pressure over $15.5 \mathrm{GPa}^{66,67}$ Due to a large energy difference between the $2 \mathrm{H}$ and $1 \mathrm{~T}^{\prime}$ phases, the metastable group VI diselenides and disulphides are challenging to obtain in a pure form and the reports on their successful synthesis are limited. ${ }^{21,68-71}$ The crystal structure of the monoclinic $1 \mathrm{~T}^{\prime}$ phase of group VI diselenides and disulphides is still debated with the possible space groups being either $P 2_{1} / m$ or $\mathrm{C} 2 / \mathrm{m}$; the latter has only been reported recently and is referred to as the $2 \mathrm{M}$ phase, where $\mathrm{M}$ stands for the monoclinic lattice system. $^{72}$ Unlike the semiconducting $2 \mathrm{H}$ counterparts, the corresponding $1 \mathrm{~T}^{\prime}$ and $\mathrm{Td}$ phases of group VI TMDs are semimetallic. $^{61}$ Furthermore, the non-centrosymmetric Td polymorph exhibits topologically nontrivial surface electronic states, ${ }^{73,74}$ and bulk Td phases of $\mathrm{WTe}_{2}$ and $\mathrm{MoTe}_{2}$ have been extensively investigated as potential candidates for the observation of 3D Weyl fermions. ${ }^{64,75-77}$ Recently, single layers of group VI TMDs with the $1 \mathrm{~T}^{\prime}$ symmetry have been predicted to host topologically protected edge states, ${ }^{46,78}$ and large QSH gaps have been verified experimentally in monolayer $1 \mathrm{~T}^{\prime} \mathrm{WTe}_{2},{ }^{19} 1 \mathrm{~T}^{\prime}$ $\mathrm{WSe}_{2},{ }^{20,21}$ and $1 \mathrm{~T}^{\prime} \mathrm{MoS}_{2}{ }^{79}$

1.3. Distinguishing the metastable group VI TMD polymorphs. Reliably distinguishing the metastable polymorphs of group VI TMDs is of paramount importance for the experimental investigation of electronic properties of the respective phases. In recent literature, the difference between $1 \mathrm{~T}$ and $1 \mathrm{~T}^{\prime}$ structural polymorphs is often neglected and the $1 \mathrm{~T}^{\prime}$ phase is frequently reported as $1 \mathrm{~T}^{80-83}$ or is more generally referred to 
a

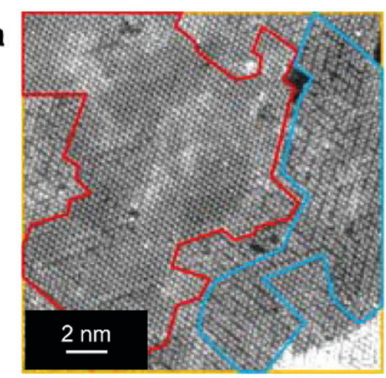

c

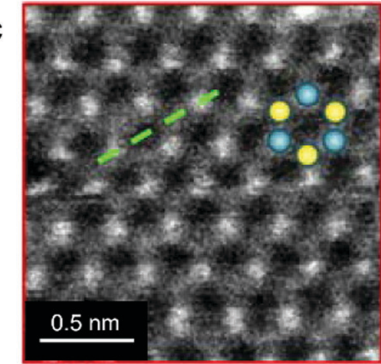

e

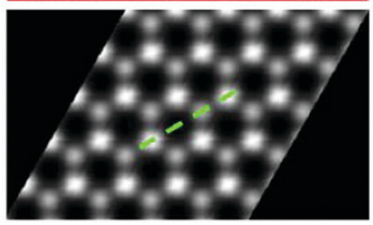

b

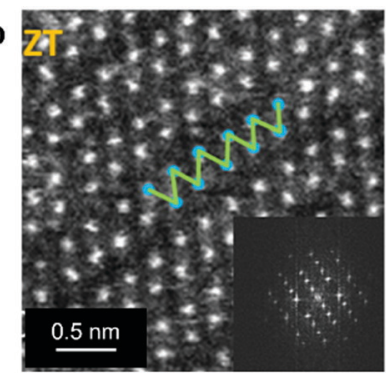

d

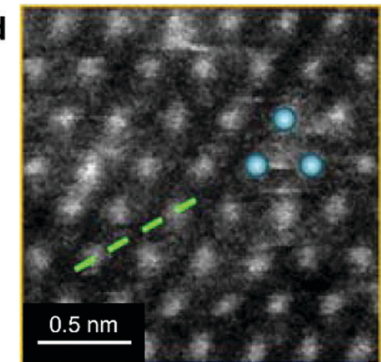

f

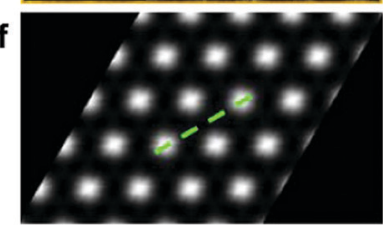

g

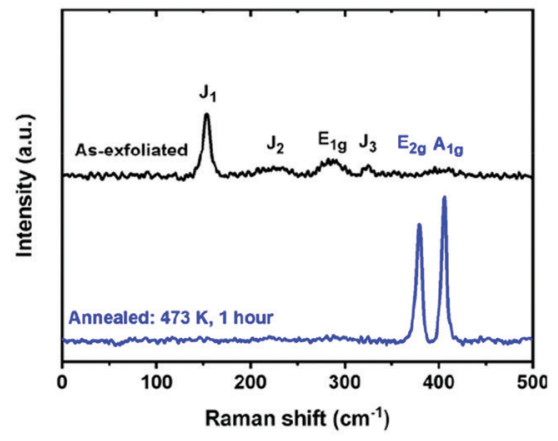

h

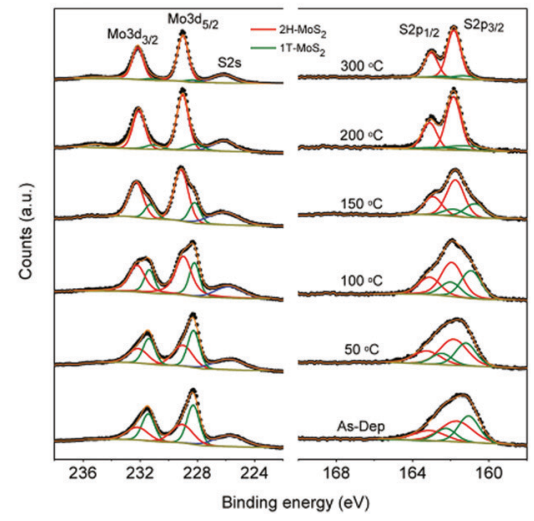

Fig. 2 Distinguishing the $1 \mathrm{H}, 1 \mathrm{~T}$, and $1 \mathrm{~T}^{\prime}$ polymorphs. (a) STEM image of chemically exfoliated MoS $\mathrm{S}_{2}$ displaying co-existing $1 \mathrm{H}, 1 \mathrm{~T}$, and $1 \mathrm{~T}^{\prime}$ phases; the corresponding areas are enclosed by red, yellow, and blue curves, respectively. (b-d) Representative HAADF STEM images of the $1 \mathrm{~T}^{\prime}, 1 \mathrm{H}$, and $1 \mathrm{~T}^{\mathrm{M}}$ MoS 2 lattice, respectively. (e and f) Simulated HAADF STEM images of the ideal $1 \mathrm{H}$ and $1 \mathrm{~T} \mathrm{MoS}_{2}$ monolayers, respectively. (g) Raman spectra of chemically exfoliated $1 \mathrm{~T}^{\prime}$ (top) and annealed $2 \mathrm{H}$ (bottom) $\mathrm{MoS}_{2}$ flakes, illustrating the characteristic vibrational modes of these two phases. (h) XPS spectra of the Mo 3d, S 2s and S 2p core level electrons of mixed-phase 1T/2H MoS nanosheets, demonstrating the gradual recovery of the $2 \mathrm{H}$ phase during thermal annealing. The $1 \mathrm{~T}$ and $2 \mathrm{H}$ phase contributions are given in green and red, respectively. Separate panels are reproduced with permission: (a and $\mathrm{c}-\mathrm{f}$ ) from ref. 85, copyright 2012, American Chemical Society; (b) from ref. 51, copyright 2016, American Chemical Society; (g) from ref. 263, copyright 2019, American Chemical Society; and (h) from ref. 264, copyright 2011, American Chemical Society.

as the metallic phase. ${ }^{84}$ The $1 \mathrm{~T}$ and $1 \mathrm{~T}^{\prime}$ domains can be unambiguously distinguished in high resolution transmission electron microscopy (HR TEM) and scanning transmission electron microscopy (HR STEM) images of atomically-thin nanosheets where the $1 \mathrm{~T}^{\prime}$ phase exhibits characteristic zigzag chains of transition metal atoms ${ }^{85,86}$ (Fig. 2a and b). However, the $1 \mathrm{~T}$ and $2 \mathrm{H}$ phases, displaying a three-fold symmetry in the $a b$ plane, are virtually undistinguishable in HR TEM images. It has recently been established that the $1 \mathrm{~T}$ and $2 \mathrm{H}$ domains in group VI TMD monolayers can be distinguished in high-angle annular dark-field (HAADF STEM) images by the intensity of chalcogen columns as shown in Fig. $2 \mathrm{c}$ and d. ${ }^{85}$ The discernible variations in signal intensity arise since in the trigonalprismatic $2 \mathrm{H}$ structure, two chalcogen atoms overlapping in the direction of the incident beam make the contrast almost comparable to that of a metal site producing a honeycomb lattice in STEM images (Fig. 2e), whereas in the octahedral $1 \mathrm{~T}$ structure, a strong contrast between transition metal and chalcogen sites results in a hexagonal lattice (Fig. 2f). Moreover, the stacking sequence of $1 \mathrm{H}$ monolayers in bi- and trilayer nanosheets can be deduced. However, extreme caution must be taken when interpreting the microscopy data and especially Fourier filtered images since residual aberrations may give rise to quasi atomic columns. ${ }^{28}$ Additionally, it is suggested that the
$2 \mathrm{H}$ and $3 \mathrm{R}$ polytypes are possible to distinguish by the intensity profiles of selected area electron diffraction (SAED) patterns taken along the [001] zone axis. ${ }^{41}$ The $\{100\}$ and $\{110\}$ diffraction spots display nearly equal intensities in the $2 \mathrm{H}$ phase, while in the $3 \mathrm{R}$ phase, their intensities differ by almost three times. ${ }^{41}$

Spectroscopic techniques, such as Raman spectroscopy and $\mathrm{X}$-ray photoemission spectroscopy (XPS), offer a relatively accurate way of detecting co-existing polymorphs in TMD nanostructures over micron-sized areas. In the original work by J. Sandoval et al., it has been reported that the octahedral $1 \mathrm{~T}$ phase of chemically exfoliated $\mathrm{MoS}_{2}$ displayed two Ramanactive modes, $\mathrm{E}_{\mathrm{g}}$ and $\mathrm{A}_{1 \mathrm{~g}}$, at 287 and $408 \mathrm{~cm}^{-1}$, respectively, while the $\mathrm{E}_{2 \mathrm{~g}}^{1}$ mode of the $2 \mathrm{H}$ phase (at $383 \mathrm{~cm}^{-1}$ ) was absent. ${ }^{87}$ In addition to those, three previously unassigned modes, $J_{1}, J_{2}$, and $J_{3}$, at 156,226 , and $333 \mathrm{~cm}^{-1}$, respectively, were found (Fig. 2g). These modes were attributed to the $2 a \times a$ superstructure in the octahedral phase. The wider set of Ramanactive modes of the $1 \mathrm{~T}^{\prime}$ and $\mathrm{Td}$ phases is indicative of their lower symmetry as compared to the $2 \mathrm{H}$ phase and is usually reported for $\mathrm{WTe}_{2}$ and $\mathrm{MoTe}_{2} \cdot{ }^{62,88-90}$ The $1 \mathrm{~T}^{\prime}$ signature $J_{1}, J_{2}$, and $J_{3}$, modes were also observed in the $1 \mathrm{~T}^{\prime} \mathrm{MoS}_{2}, \mathrm{WS}_{2}, \mathrm{MoSe}_{2}$, and $\mathrm{WSe}_{2}$ single crystals ${ }^{68,91}$ and few-layered nanosheets. ${ }^{71,86,92,93}$ It should be noted that the $J_{1}$ mode consistently exhibits the highest intensity in Raman spectra of nearly phase-pure $1 \mathrm{~T}^{\prime}$ group 


\section{Destabilisation of the thermodynamically stable phase}

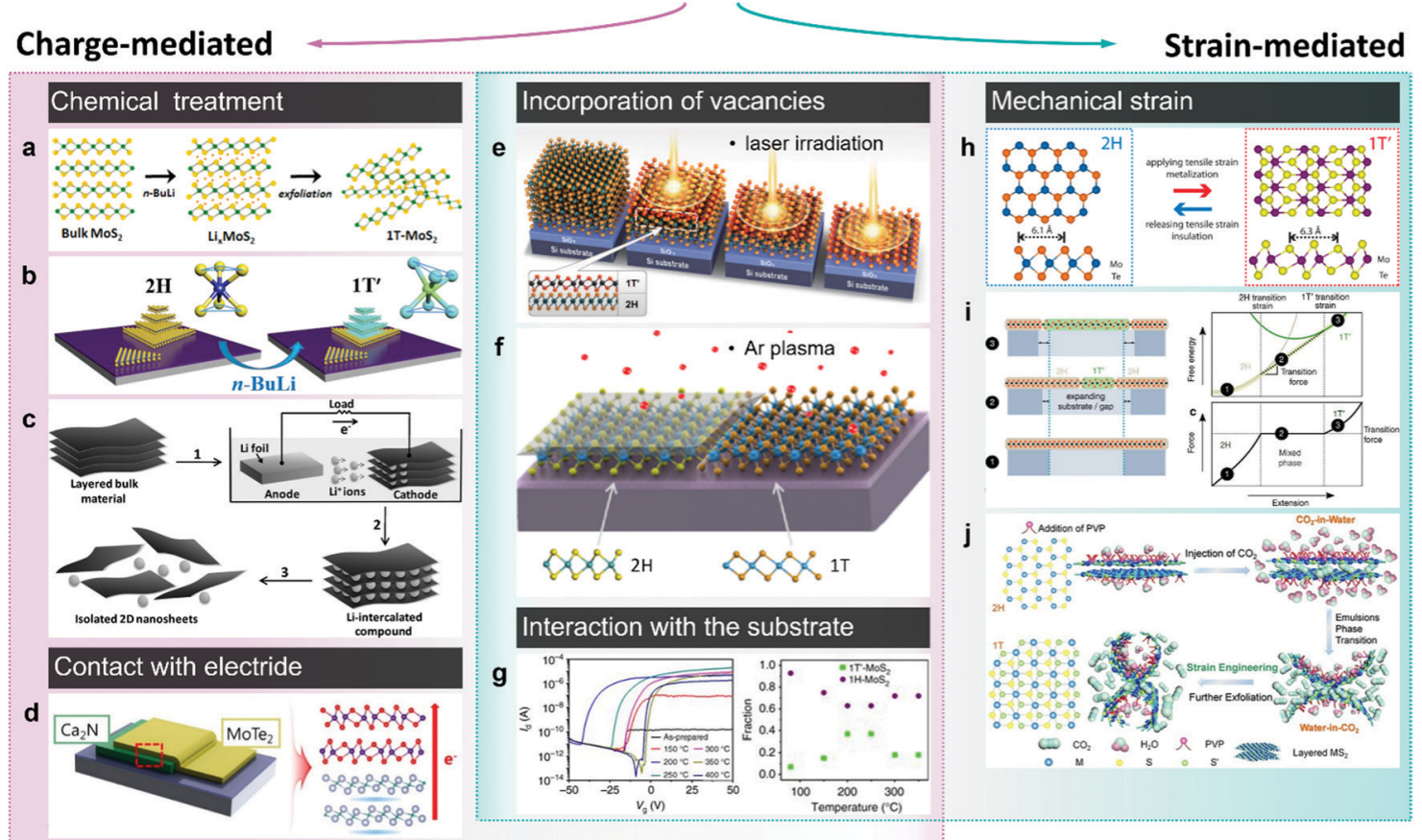

Fig. 3 Accessing the metastable $1 \mathrm{~T}\left(1 \mathrm{~T}^{\prime}\right)$ phase via destabilisation of the thermodynamically stable $(2 \mathrm{H})$ phase. The approaches to induce the $2 \mathrm{H}$-to-1 $1 \mathrm{~T}^{\prime}$ transition in group VI TMDs are divided in two categories: charge-mediated, as chemical treatment (panels a-c) and contact with an electride (panel d); and strain-mediated, such as tensile strain-induced transition between the $2 \mathrm{H}$ and $1 T^{\prime}(1 \mathrm{~T})$ polymorphs (panels $\mathrm{h}-\mathrm{j}$ ). Panels e-g represent the phase transitions caused by the combination of charge and strain as in the case of chalcogen vacancies (panels e and f) or due to the interaction with the substrate (panel g). Separate panels are adapted with permission: (a) from ref. 100, copyright 2017, American Chemical Society; (b) from ref. 114, copyright 2018, American Chemical Society; (c) from ref. 108, copyright 2011, John Wiley \& Sons; (d) from ref. 123, copyright 2017, American Chemical Society; (e) from ref. 155, copyright 2015, American Association for the Advancement of Science; (f) from ref. 138, copyright 2017, American Chemical Society; (g) from ref. 128, copyright 2017, Springer Nature; (h) from ref. 130, copyright 2016, American Chemical Society; (i) from ref. 25, copyright 2014, Springer Nature; and (j) from ref. 134, copyright 2017, John Wiley \& Sons.

VI disulphides. ${ }^{86,92,93}$ Further, the overall Raman signal intensity is significantly lower in the $1 \mathrm{~T}\left(1 \mathrm{~T}^{\prime}\right)$ phase $\mathrm{TMDs}$ than in the $2 \mathrm{H}$ samples of equivalent thickness as has been experimentally demonstrated in the case of $\mathrm{MoS}_{2}{ }^{94-96}$ and $\mathrm{WSe}_{2}$ nanosheets. ${ }^{71,97}$

In XPS spectra, both the $1 \mathrm{~T}$ and $1 \mathrm{~T}^{\prime}$ phases are reported to present peaks systematically shifted to lower binding energies as compared to the $2 \mathrm{H}$ phase, however, XPS is not sensitive to the differences between the $1 \mathrm{~T}$ and $1 \mathrm{~T}^{\prime}$ phases $^{98}$ (Fig. 2h). In chemically exfoliated TMDs, the shift to lower binding energies is typically ascribed to partial reduction of transition metal centres during alkali metal intercalation. ${ }^{91}$

\section{Access to the metastable crystal phases of group VI TMDs}

The metastable crystal phases can be either accessed through destabilisation of the thermodynamically stable polymorphs, for instance, due to electron transfer, or synthesised directly, when the growth conditions are optimised in such a way that the metastable product is kinetically preferred.

\subsection{H-to-1T $\left(\mathbf{1 T}^{\prime}\right)$ phase transformation}

2.1.1. Chemical treatment. The first reports on the metallic $1 \mathrm{~T}\left(1 \mathrm{~T}^{\prime}\right)$ group VI TMD polymorphs date back to the pioneering works by R. Frindt et al. on chemical exfoliation of these materials ${ }^{27,50}$ (Fig. 3a). Chemical exfoliation of layered materials typically proceeds in two steps. ${ }^{4,50,99}$ At first, bulk TMD powders are intercalated with alkali metals. The most commonly used intercalants include $n$-BuLi ${ }^{83,100,101} t$-BuLi ${ }^{102}$ and $\mathrm{LiBH}_{4} \cdot{ }^{50,103}$ The subsequent hydrolysis of the intercalated alkali metal cations leads to the generation of hydrogen gas bubbles that split the individual layers apart. The exfoliation can be facilitated by gentle shaking or sonication. It has been demonstrated that upon intercalation of alkali metal species between the layers of bulk crystals of group VI TMDs, the crystal phase would change from the $2 \mathrm{H}$ to $1 \mathrm{~T}$. It has been suggested that the mechanism of this structural transition should be imputed to the electron transfer from the intercalated alkali metal species leading to an increased electron count on the transition metal d-orbitals. ${ }^{47}$ Specifically, for the case of $\mathrm{MoS}_{2}$ monolayers, it has been shown that the injection of 4 electrons per unit cell allows lowering the $2 \mathrm{H}$-to-1T transition barrier from 1.59 to only $0.27 \mathrm{eV}{ }^{48}$ Thus, upon this electron transfer (chemical reduction), the octahedral coordination of transition metal becomes favoured over the trigonal-prismatic and thus the $2 \mathrm{H}$-to- $1 \mathrm{~T}$ structural phase transition occurs. ${ }^{104}$ In the absence of intercalated species, the $2 \mathrm{H}$-to1T phase transition due to accumulation of negative charges was also observed in Re doped $\mathrm{MoS}_{2}$ monolayers under continuous electron beam scanning during STEM imaging. ${ }^{42}$ The transition occurs through the initial formation of numerous non-parallel molybdenum zigzag chains which is followed by chalcogen plane 


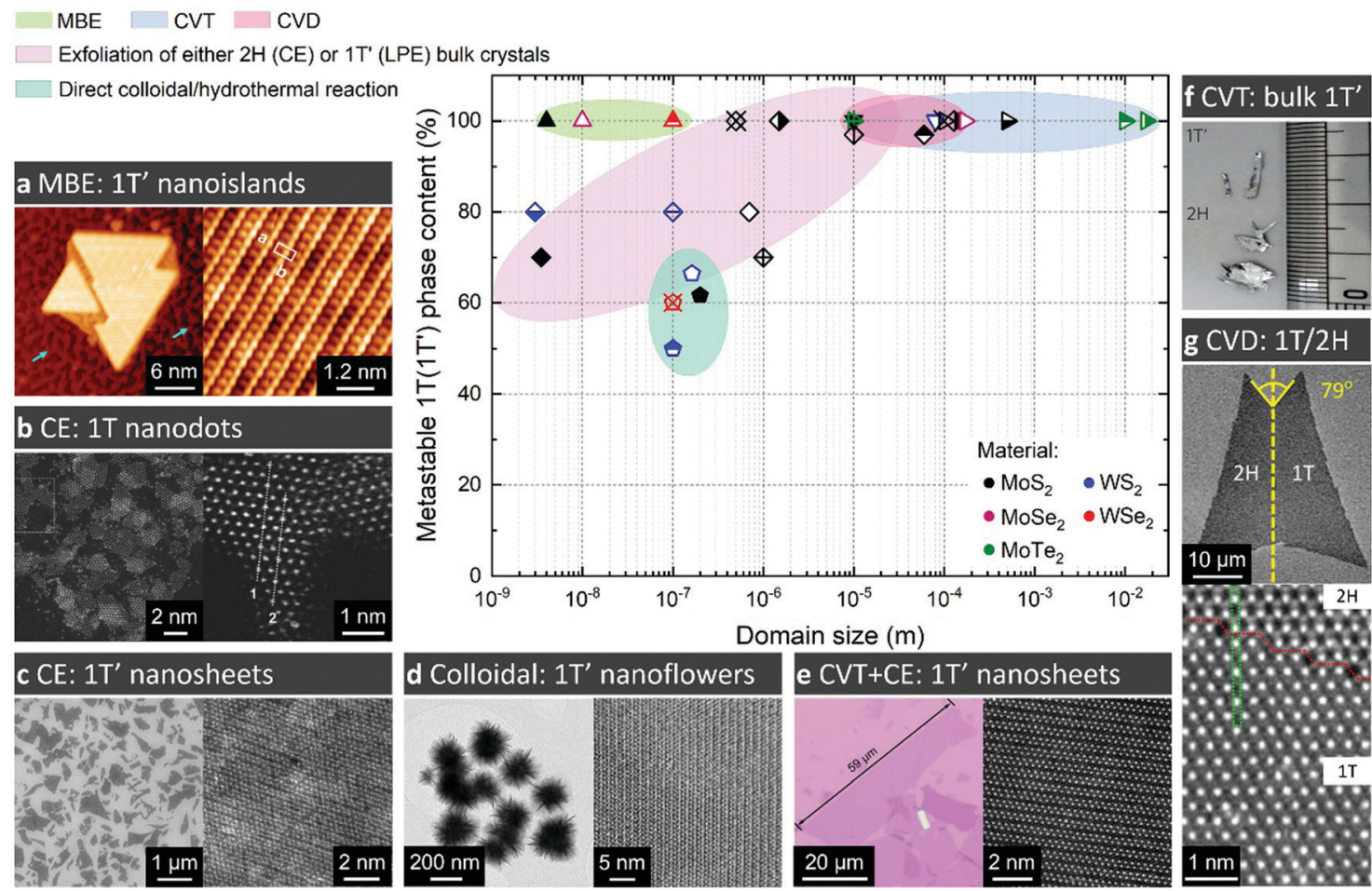

Fig. 4 Comparison of various experimental approaches to produce the metastable $1 T\left(1 T^{\prime}\right)$ phases of group VI TMDs. The approaches are grouped into the following categories: molecular beam epitaxy (MBE); chemical vapour transport (CVT) growth; chemical vapour deposition (CVD); chemical (CE) or liquid-phase (LPE) exfoliation of bulk crystals; and direct synthesis approaches, such as colloidal and hydrothermal growth. The experimental data are taken from the following articles: ref. 21, 56, 58, 68-71, 79-81, 83, 93, 101, 103, 112, 144, 147, 148, 153, 162 and 164. Separate panels are adapted with permission: (a) from ref. 70, copyright 2019, American Chemical Society; (b) from ref. 112, copyright 2018, John Wiley \& Sons; (c) from ref. 103, copyright 2013, American Chemical Society; (d) from ref. 71, copyright 2019, Springer Nature; (e) from ref. 69, copyright 2019, John Wiley \& Sons; (f) from ref. 153, copyright 2015, Springer Nature; and (g) from ref. 164, copyright 2018, American Chemical Society.

gliding, reducing mechanical strain and forming a domain of the trigonal $1 \mathrm{~T}$ phase. ${ }^{42}$

Generally, the $1 \mathrm{~T}\left(1 \mathrm{~T}^{\prime}\right)$ phases produced via chemical exfoliation approach are attained as small crystalline (within the range 1-10 nm, Fig. 4) domains often embedded into the $2 \mathrm{H}$ matrix ${ }^{85}$ (Fig. 2a and 4c). Patches of both $1 \mathrm{~T}$ and $1 \mathrm{~T}^{\prime}$ phases are found in chemically exfoliated $\mathrm{MoS}_{2}$ nanosheets. ${ }^{49,85,101}$ Whereas a characteristic distortion in the $a b$ plane of chemically exfoliated $\mathrm{WS}_{2}$ indicates the crystal phase to be $1 \mathrm{~T}^{\prime}{ }^{47,51}$ although the resulting crystal phase is commonly denoted as $1 \mathrm{~T} .^{83}$ The ratio between the converted $1 \mathrm{~T}\left(1 \mathrm{~T}^{\prime}\right)$ and pristine $2 \mathrm{H}$ phases strongly depends on the type and concentration of reducing agent (alkali metal) and the TMD material. ${ }^{102}$ The maximum amount of $1 \mathrm{~T}\left(1 \mathrm{~T}^{\prime}\right)$ phases achievable via chemical exfoliation approach is reported to be $\sim 70-80 \%$ of the overall material ${ }^{92,101}$ (typically, estimated from by XPS data, Fig. 4). The metastable $1 \mathrm{~T}\left(1 \mathrm{~T}^{\prime}\right)$ phases obtained by chemical exfoliation are reported to gradually decay into the $2 \mathrm{H}$ polymorph over time; this process is considerably promoted in the restacked films once the stabilising ions have been removed during drying. ${ }^{49,87,105}$ A full conversion to the $2 \mathrm{H}$ phase occurs if the material is subjected to high temperatures, such as $\sim 100{ }^{\circ} \mathrm{C}$ for $\mathrm{MoS}_{2}{ }^{27}$ and $\sim 200{ }^{\circ} \mathrm{C}$ for $\mathrm{WS}_{2} \cdot{ }^{50}$

Recently, it has been demonstrated that the lifetime of the $1 \mathrm{~T}\left(1 \mathrm{~T}^{\prime}\right)$ phases in intercalated few-layered $\mathrm{MoS}_{2}$ flakes can be considerably extended via the hydrogenation of intercalated lithium species. ${ }^{86}$ Results of DFT calculations suggest that $\mathrm{LiH}$ is a good electron donor that stabilises the $1 \mathrm{~T}^{\prime}$ phase in multilayered flakes for over 3 months. Although $\mathrm{LiH}$, encapsulated between the TMD layers, is stable, the surface bound lithium hydride can easily convert into $\mathrm{Li}_{2} \mathrm{CO}_{3}$ and $\mathrm{Li}_{3} \mathrm{~N}$, what makes this approach unsuitable for stabilisation of the $1 \mathrm{~T}^{\prime}$ phase in chemically exfoliated single layers.

A morphological aspect should be also taken into consideration as the non-uniform distribution of intercalated lithium hinders the control over the dimensions and distribution of the converted $1 \mathrm{~T}\left(1 \mathrm{~T}^{\prime}\right)$ patches. The intercalation kinetics affects the conversion efficiency and the lateral dimensions of the patches of metastable phase. ${ }^{106,107} \mathrm{~A}$ uniform lithiation has been reported by an electrochemical intercalation process where $\mathrm{Li}$ foil serves as an anode and bulk TMD material as a cathode ${ }^{108}$ (Fig. 3c). After lithium insertion, lithiated powders are exfoliated by mild sonication in water producing predominantly monolayered nanosheets with lateral dimensions of up to $1 \mu \mathrm{m}$. Moreover, it has been demonstrated that the lithium migration within the van der Waals (vdW) gaps of lithiated few-layered material can be controlled by an external electric field so that a reversible $2 \mathrm{H}$-to- $1 \mathrm{~T}^{\prime}$ phase transformation can be uniformly extended over micron-sized areas. ${ }^{109}$ Such controllable phase switching has been demonstrated in lithiated $\mathrm{MoS}_{2}$ thin films, 
which have been used as neuromorphic memory devices, ${ }^{109}$ and in ultra-thin $(\sim 2-5 \mathrm{~nm}) \mathrm{MoS}_{2}$ nanosheets upon a reversible electrochemical lithiation. ${ }^{110}$ Another method to achieve a uniform intercalation in group VI TMDs has been recently reported by J. Zheng et al. ${ }^{111}$ Performed prior to intercalation, the expansion of TMD powders by reacting with hydrazine under hydrothermal conditions has facilitated the alkali metal naphthalenide insertion. This allowed producing single layer (80\%) $\mathrm{MoS}_{2}$ sheets of up to $400 \mu \mathrm{m}^{2}$ in size; the metastable $1 \mathrm{~T}\left(1 \mathrm{~T}^{\prime}\right)$ phase content, however, was not specified. ${ }^{111}$

It is interesting to note that combined with ball-milling of $2 \mathrm{H}$-phase bulk crystals, chemical (organolithium) treatment and exfoliation approach has recently been shown to produce $\mathrm{MoS}_{2}, \mathrm{MoSe}_{2}, \mathrm{WS}_{2}, \mathrm{Mo}_{0.5} \mathrm{~W}_{0.5} \mathrm{~S}_{2}$ and MoSSe nanodots with lateral sizes of $2-5 \mathrm{~nm}$ and a high $(70 \%)$ content of the metastable $1 \mathrm{~T}$ phase ${ }^{112}$ (Fig. 4b).

Phase conversion can also be achieved via a post-growth treatment of CVD grown 2H TMD few-layered flakes and films with solutions of $n$-BuLi in nonpolar solvents ${ }^{113,114}$ (Fig. 3b). Considering that CVD grown TMD flakes present much larger (tens of microns) lateral sizes compared to the ones of chemically exfoliated flakes (submicron for monolayers), this could allow for the production of larger continuous domains of the metastable $1 \mathrm{~T}\left(1 \mathrm{~T}^{\prime}\right)$ phases. The $n$-BuLi treatment of polycrystalline films of $2 \mathrm{H}-\mathrm{MoS}_{2}$ and $2 \mathrm{H}-\mathrm{MoSe}_{2}$ grown on $\mathrm{Si} / \mathrm{SiO}_{2}$ wafers led to a partial conversion into the metallic $1 \mathrm{~T}$ phase, with an overall conversion efficiency of $\sim 50 \% .{ }^{113}$ Further, the lithiuminduced structural transformations in the CVD grown $2 \mathrm{H}-\mathrm{MoS}_{2}$ flakes appear to be thickness-dependent. ${ }^{114}$ The increased energy barrier of the $2 \mathrm{H}$-to-1T phase transition in $\mathrm{MoS}_{2}$ monolayers implies much longer treatment time in order to achieve the phase conversion compared to the multi-layered counterparts. ${ }^{114}$ This, therefore, paves the way to the $2 \mathrm{D}$ metal-semiconductor junctions in CVD grown TMD devices by controlling the exposure to lithiating agent ( $n$-BuLi). In the case of $2 \mathrm{H}-\mathrm{WSe}_{2}$ monolayers, a complete conversion into the metallic $1 \mathrm{~T}$ phase has been reported and the deposition of a PMMA mask has allowed for the patterning of $2 \mathrm{H} / 1 \mathrm{~T}$ heterojunctions. ${ }^{97}$ While a complete conversion to the $1 \mathrm{~T}\left(1 \mathrm{~T}^{\prime}\right)$ phases via lithium intercalation of bulk $\mathrm{MoSe}_{2}$ and $\mathrm{WSe}_{2}$ powders have not been achieved. ${ }^{102,115}$ Finally, the $2 \mathrm{H}$-to- $1 \mathrm{~T}$ phase conversion has been demonstrated to be induced also by exposure to the vapours of electron donating compounds, such as butylamine and trimethylamine, as was shown in the cases of $\mathrm{MoS}_{2}$ and $\mathrm{MoSe}_{2}{ }^{116}$ It has been proposed that this approach can be potentially employed to fabricate new passive sensing devices based on detecting the abrupt changes in material conductivity in the presence of strong donor analytes, such as nerve gas.

2.1.2. Electrostatic gating. The dynamical control of the structural phase transitions in TMD monolayers by an applied electric field can be potentially interesting for application in light-weight flexible electronic devices, including non-volatile memory devices. ${ }^{117}$ Bias-voltage driven T-to-H crystal phase transformations have been previously demonstrated in $\mathrm{TaS}_{2}$ crystals. ${ }^{118} \mathrm{Y}$. Li et al. have proposed that the semiconductor-to- semimetal phase transition can be achieved in some group VI TMDs using an electrostatic gating. ${ }^{119}$ It has been found that the transition between the $2 \mathrm{H}$ and $1 \mathrm{~T}^{\prime}$ phases can be driven by excess electric charge in the monolayer. Thus, the excess charge densities less than $-0.04 e$ or greater than $0.09 e$ per formula unit would suffice to induce the $2 \mathrm{H}$-to- $1 \mathrm{~T}^{\prime}$ transformation in an undoped $\mathrm{MoTe}_{2}$ monolayer; however, to achieve this transition in lighter TMD monolayers, such as $\mathrm{MoS}_{2}$ and $\mathrm{WS}_{2}$, much greater excess charge densities are needed. Alternatively, a constant voltage of -1.6 or $4.4 \mathrm{~V}$ is required to induce the semiconductor-to-semimetal transition in monolayer $\mathrm{MoTe}_{2}$ on $\mathrm{HfO}_{2}$ in the electrostatic gating device configuration. ${ }^{119}$ A reversible $2 \mathrm{H}$-to- $1 \mathrm{~T}^{\prime}$ phase transition was achieved in $\mathrm{MoTe}_{2}$ by ionic liquid gating in a field-effect transistor configuration. ${ }^{120}$ The required gate voltages could be substantially reduced by varying the chemical composition, such as by alloying $^{119,121}$ or forming Janus monolayers. ${ }^{122}$

\subsubsection{Electron doping by contacting with an electron reservoir.} Long-range crystal lattice modulation can be achieved by contacting the $2 \mathrm{H}$ TMD $\left(\mathrm{MoTe}_{2}\right)$ with a single crystalline 2D electride as has been demonstrated in ref. 123 (Fig. 3d). Electrides are a class of inorganic compounds in which electrons serve as anions. ${ }^{124}$ Layered electrides containing two-dimensionally confined anionic electrons are generally characterised by exceptionally high charge carrier concentrations $\left(1.4 \times 10^{22} \mathrm{~cm}^{-3}\right)$ and high electron mobilities at room temperature $\left(160 \mathrm{~cm}^{2} \mathrm{~V}^{-1} \mathrm{~s}^{-1}\right) \cdot{ }^{125}$ Similarly to van der Waals solids, layered electrides can be exfoliated into 2D sheets. ${ }^{126}$ Unlike the chemical treatment discussed earlier, electron transfer from the electride allows for achieving higher levels of doping (about $10^{14} \mathrm{~cm}^{-2}$ ). Such high doping densities can also be achieved by ionic modulation, however, contacting with electride is effective over larger distances from the contact interface. For instance, forming a vertical heterostructure between $\left[\mathrm{Ca}_{2} \mathrm{~N}\right]^{+} \cdot \mathrm{e}^{-}$and few-layered $\mathrm{MoTe}_{2}$ has led to a uniform $2 \mathrm{H}$-to-1 $1 \mathrm{~T}^{\prime}$ phase transformation over a micron sized area even as far away as $100 \mathrm{~nm}$ from the electride. ${ }^{123}$

A reversible $2 \mathrm{H}$-to- $1 \mathrm{~T}$ phase transition has been demonstrated in $\mathrm{MoS}_{2}$ monolayers by an injection of hot electrons generated by plasmon excitation in Au nanospheres. ${ }^{127}$ Beyond that, electron injection from gold substrate was reported to cause the $1 \mathrm{H}$-to- $1 \mathrm{~T}^{\prime}$ phase transition in $\mathrm{MoS}_{2}$ single layers during thermal annealing leading to the formation of co-existing domains of $1 \mathrm{H}$ and $1 \mathrm{~T}^{\prime}$ phases; this electron-driven transition was facilitated by the interfacial strain ${ }^{128}$ (Fig. 3g). The $1 \mathrm{~T}^{\prime}$ phase content reached its maximum (37\%) at the annealing temperature about $200-250{ }^{\circ} \mathrm{C}$.

2.1.4. Applying mechanical strain. According to density functional calculations, group VI TMDs are predicted to undergo a transition from the $2 \mathrm{H}$ to the $1 \mathrm{~T}^{\prime}$ phase under tensile strain. ${ }^{25}$ It is identified that a $0.3-3 \%$ uniaxial strain applied along the $b$ (armchair) direction of $2 \mathrm{H} \mathrm{MoTe}{ }_{2}$ monolayers allows for the transition into the $1 \mathrm{~T}^{\prime}$ phase at room temperature ${ }^{25}$ (Fig. 3i). The strain-induced $2 \mathrm{H}$-to- $1 \mathrm{~T}^{\prime}$ phase transition in $\mathrm{MoTe}_{2}$ monolayers is presumed to occur due to simultaneous transition 
metal and tellurium atoms displacement and does not proceed through an intermediate $1 \mathrm{~T}$ state. ${ }^{129}$ The process involves a distortion of the transition metal sublattice accompanied by a simultaneous gliding and rippling of chalcogen plane. ${ }^{129}$ Experimentally, a reversible straininduced $2 \mathrm{H}$-to- $1 \mathrm{~T}^{\prime}$ phase transition has been demonstrated in $20 \mathrm{~nm}$ thick $\mathrm{MoTe}_{2}$ films by applying a tensile strain using an AFM tip ${ }^{130}$ (Fig. 3h). Notably, such a phase transition was realised even at room temperature if a small tensile strain of $0.2 \%$ was applied.

Among the emerging approaches to induce the $2 \mathrm{H}$-to-1T phase transformation in group VI TMDs, we would like also to mention the microwave plasma treatment ${ }^{131,132}$ and the supercritical $\mathrm{CO}_{2}$ treatment. ${ }^{133,134}$ These less conventional approaches allow for a controllable phase engineering also in the liquid phase. Recently, X. Tong et al. have discovered that during the supercritical $\mathrm{CO}_{2}$ treatment, aqueous suspensions of TMD nanosheets transform into water-in- $\mathrm{CO}_{2}$ emulsions, causing a strain-induced $2 \mathrm{H}$-to- $1 \mathrm{~T}$ reconstruction in $\mathrm{WS}_{2}$ and $\mathrm{MoS}_{2}$ flakes confined at the convex water/CO $\mathrm{CO}_{2}$ interface ${ }^{134}$ (Fig. 3j). The metastable 1T phase content achieved by this approach is reported to vary from $70 \%{ }^{133}$ to $90 \% .{ }^{134}$ Similar to supercritical $\mathrm{CO}_{2}$ treatment, mild microwave plasma treatment has been reported to introduce the $1 \mathrm{~T}$ phase domains in the exfoliated $2 \mathrm{H} \mathrm{MoS}$ nanosheets. ${ }^{132} \mathrm{C}$. Sharma et al. have used forming gas microwave plasma to convert exfoliated $2 \mathrm{H} \mathrm{MoS}$ flakes into the $1 \mathrm{~T}$ phase with a $70 \%$ efficiency. The phase transition is assumed to proceed due to the momentum transfer from the plasma ions to the $\mathrm{MoS}_{2}$ lattice, causing the chalcogen plane gliding. ${ }^{131}$

2.1.5. The effect of chalcogen vacancies. Another type of electron donors are the chalcogen vacancies formed in TMD nanosheets. The presence of chalcogen vacancies plays a key role in promoting the $2 \mathrm{H}$-to- $1 \mathrm{~T}$ phase transition by a dual mechanism: generation of extra charges and weakening of the metal-chalcogen bonds. Both facilitate the structural transformation. ${ }^{135}$ Specifically, in $\mathrm{MoS}_{2}$, the difference in the formation energy between the $2 \mathrm{H}$ and $1 \mathrm{~T}$ phase decreases almost to zero when the vacancy concentration reaches 8 at $\% .{ }^{136}$ Acting as point defects, the vacancies also serve as nucleation sites of the $1 \mathrm{~T}^{\prime}$ phase being formed in the $2 \mathrm{H}$ matrix.

Local $2 \mathrm{H}$-to- $1 \mathrm{~T}^{\prime}$ transition occurring in mechanically exfoliated few-layered $\mathrm{MoTe}_{2}$ flakes under laser irradiation is commonly attributed to the formation of Te vacancies due to local heating ${ }^{137}$ (Fig. 3e). This transition is irreversible; however, the $2 \mathrm{H}$ phase can be restored by high temperature annealing in tellurium atmosphere. ${ }^{137}$

In the case of $\mathrm{MoS}_{2}$, it has been shown that the chalcogen vacancies can be introduced into the material by gentle $\mathrm{Ar}$ plasma treatment ${ }^{138}$ (Fig. 3f). The phase transformation was demonstrated to occur in the vicinity of single point defects and the lateral sizes of the $1 \mathrm{~T}^{\prime} \mathrm{MoS}_{2}$ domains were typically about a few nm. ${ }^{138}$ Combined with shadow mask technique, lateral $2 \mathrm{H} / 1 \mathrm{~T}^{\prime}$ homojunctions, including $1 \mathrm{D}$ conducting $1 \mathrm{~T}^{\prime}$ channels, were patterned by Ar plasma treatment in $\mathrm{MoS}_{2}$ monolayers. ${ }^{139}$
A controlled laser beam $2 \mathrm{H} / 1 \mathrm{~T}^{\prime}$ patterning has also been performed in few-layered $\mathrm{MoS}_{2}$ flakes. ${ }^{140}$ However, Ar plasma treatment might be preferable for the phase patterning on TMD monolayers since this method is considered less disruptive compared to laser beam irradiation. ${ }^{139}$ Other methods of introducing chalcogen vacancies in $\mathrm{MoS}_{2}$, as for example harsh electrochemical etching, to cause the $2 \mathrm{H}$-to-1T structural transformation are just emerging. ${ }^{135}$ In their recent work, X. Gan et al. have demonstrated that the $1 \mathrm{~T}$ phase content in multilayered $\mathrm{MoS}_{2}$ can be varied by either increasing the etching potential or the reaction time, although the spatial distribution of the metastable phase is not possible to control. ${ }^{135}$

2.2. Direct growth of the metastable $\mathbf{1 T}^{\prime}$ phase of group VI TMDs. Direct growth techniques, such as vapour-phase deposition and solution-phase reactions, are based on chemical reactions between the constituent precursors and commonly enable a good level of control of the synthetic conditions. The methods to obtain the metastable $1 \mathrm{~T}\left(1 \mathrm{~T}^{\prime}\right)$ phase of group VI TMDs presented in literature can be divided into two categories: the first rely on the formation of the metastable crystal phase due to the interaction with charged reaction by-products or with the substrate; while the second rely on optimising the reaction kinetics in such a way that the kinetically-preferred, yet thermodynamically metastable, product is formed.

2.2.1. The role of a substrate in a crystal phase-selective growth. The use of engineered substrates has been found beneficial for growing the metastable phases of materials with large energy difference between the stable and metastable polymorphs (550 and $330 \mathrm{meV}$ per formula unit in $\mathrm{MoS}_{2}$ and $\mathrm{MoSe}_{2}$, respectively ${ }^{25}$ ). Nanometre-sized $1 \mathrm{~T}^{\prime} \mathrm{MoSe}_{2}$ islands were selectively formed on $\mathrm{Au}(111)$ substrates which were treated with selenium prior to the deposition, whereas only the thermodynamically stable $1 \mathrm{H}$ polymorph was obtained if the layer of selenium was not pre-deposited ${ }^{70}$ (Fig. 4a). Such a difference in the resulting crystal phase was attributed to the formation of an electron donating Mo layer between the $\mathrm{Au}$ surface and the pre-sputtered Se overlayer that facilitate the growth of the $1 \mathrm{~T}^{\prime}$ phase. MBE deposition of $1 \mathrm{~T}^{\prime} \mathrm{MoS}_{2}$ nanoclusters (30-50 atoms) on $\mathrm{Au}(111)$ substrates has also been reported. ${ }^{79}$ It has been also hypothesized that the gold substrate itself serves as an electron reservoir that stabilised the nucleating $1 \mathrm{~T}^{\prime}$ phase.

Thermal expansion coefficients of various substrates might be another important growth parameter to consider when the formation of a metastable phase is targeted. Typically, the formation of metastable polymorphs of $\mathrm{MoS}_{2}$ does not occur in a direct chemical vapour deposition (CVD) reaction due to the huge energy barrier for the $1 \mathrm{H}$-to-1T phase transition. Recently, Z. Wang et al. reported the direct growth of the $1 \mathrm{~T}-1 \mathrm{H}$ bi-phase TMD monolayers, in which the formation of the metastable phase is associated with the in-plane thermal strain imposed by the substrate during the cooling step. ${ }^{141}$

2.2.2. Synthesis from electron donating precursors. High phasepurity mm-sized $1 \mathrm{~T}$ and $1 \mathrm{~T}^{\prime}$ phase $\mathrm{MoS}_{2}$ single crystals have been demonstrated through a delicate deintercalation of $\mathrm{Li}$ or $\mathrm{K}$ 


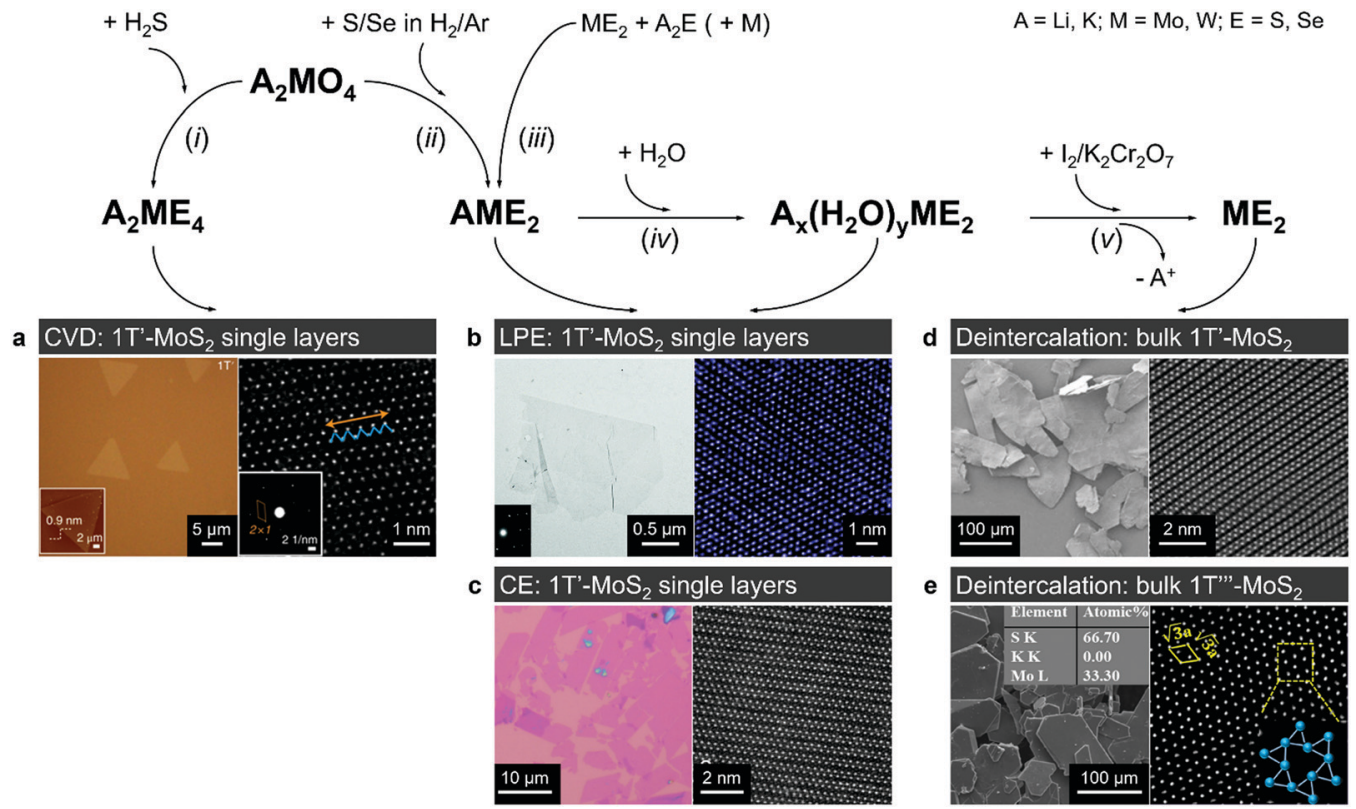

Fig. 5 Direct vapour-phase (CVT, CVD) growth of the $1 T\left(1 T^{\prime}\right)$ polymorphs from alkali metal containing precursors. Bulk $1 T^{\prime}$ crystals can be produced by gentle deintercalation of bulk intercalation compounds $A_{x}\left(\mathrm{H}_{2} \mathrm{O}\right)_{y} \mathrm{ME}_{2}$ (panels d and e); $1 \mathrm{~T}^{\prime}$ monolayers can be obtained by chemical (CE, panel c) or liquid-phase (LPE, panel b) exfoliation of bulk intercalation compounds $A_{x}\left(\mathrm{H}_{2} \mathrm{O}\right)_{y} M E_{2}$ and $A M E_{2}$; high-quality $1 T^{\prime}$ monolayers can be grown by $C V D$ method from $\mathrm{A}_{2} \mathrm{ME}_{4}$ precursor (panel a). Separate panels are adapted with permission: (a) from ref. 147, copyright 2018, Springer Nature; (b) from ref. 144, copyright 2017, The Royal Society of Chemistry; (c) from ref. 69, copyright 2019, John Wiley \& Sons; (d) from ref. 68, copyright 2018, Springer Nature; and (e) from ref. 56, copyright 2019, American Chemical Society.

ions from $\mathrm{LiMoS}_{2}$ and $\mathrm{KMoS}_{2}$ compounds. ${ }^{27,69,142,143}$ Ternary $\mathrm{KMOS}_{2}$ is typically produced by reacting the corresponding alkali metal molybdate, $\mathrm{K}_{2} \mathrm{MoO}_{4}$, with dry $\mathrm{H}_{2} \mathrm{~S}$ that is then followed by a mild reduction in $\mathrm{H}_{2} / \mathrm{N}_{2}$ atmosphere ${ }^{27,142}$ (Fig. 5, reactions $\mathrm{i}$ and ii). Alternatively, $\operatorname{LiMoS}_{2}$ and $\mathrm{KMoS}_{2}$ can be obtained by fusing $\mathrm{MoS}_{2}$ with either $\mathrm{Li}_{2} \mathrm{~S}$ or $\mathrm{K}_{2} \mathrm{~S}$ under vacuum in the presence of metallic $\mathrm{Mo}^{56,143,144}$ (Fig. 5, reaction iii). The deintercalation of these alkali metal thiomolybdate precursors is reported to proceed in two stages. At first, the ternary compounds are exposed to water to hydrate the alkali metal ions intercalated between $\mathrm{MoS}_{2}$ layers ${ }^{143}$ (Fig. 5, reaction iv):

$$
\mathrm{LiMoS}_{2}+\mathrm{H}_{2} \mathrm{O}=\mathrm{Li}_{1-x}\left(\mathrm{H}_{2} \mathrm{O}\right)_{y} \mathrm{MoS}_{2}+x \mathrm{LiOH}+x / 2 \mathrm{H}_{2} .
$$

This leads to the lattice expansion in the $c$ direction. It is assumed that the increased interlayer distance facilitates the alkali metal ions removal owing to the reduced interaction with the chalcogen atoms of basal planes. ${ }^{143}$ The alkali metal is removed by a treatment with a mild oxidiser, such as $\mathrm{I}_{2}{ }^{27,69}$ (Fig. 5 , reaction v):

$$
\mathrm{Li}_{1-x}\left(\mathrm{H}_{2} \mathrm{O}\right)_{y} \mathrm{MoS}_{2}+(1-x) / 2 \mathrm{I}_{2}=\mathrm{MoS}_{2}+(1-x) \mathrm{LiI}+\mathrm{H}_{2} \mathrm{O} \text {. }
$$

Using lithiated compounds might be preferable since smaller ionic radius of lithium leads to its more effective hydration and complete removal from the interlayer gaps, while the deintercalation of larger potassium ions is believed to cause numerous lattice defects. ${ }^{142,145}$ It has been reported that the structural disorder in the intercalation compounds of $\mathrm{MoS}_{2}$ is externally stabilised by hydrated ions trapped between the layers. ${ }^{146}$ Thus, $\mathrm{K}_{0.7} \mathrm{MoS}_{2}$, similarly to $\mathrm{Li}_{x} \mathrm{MoS}_{2}(x \leq 1)$, typically demonstrates the $2 a \times 2 a$ superstructure, ${ }^{145}$ whereas the structure of the hydrated $\mathrm{K}_{x}\left(\mathrm{H}_{2} \mathrm{O}\right)_{y} \mathrm{MoS}_{2}$, refined by diffraction methods (XRD, SAED) and STM imaging, is assigned to the monoclinic symmetry, similar to the one reported for $\mathrm{WTe}_{2}(2 a \times a) \cdot{ }^{142,146}$ Depending on the starting composition of the intercalation compound, different distorted phases of $\mathrm{MoS}_{2}$ were demonstrated. ${ }^{146}$ Gentle deintercalation of $\mathrm{Li}_{x}\left(\mathrm{H}_{2} \mathrm{O}\right)_{y} \mathrm{MoS}_{2}$ with iodine solution was shown to result the high-quality $1 \mathrm{~T} \mathrm{MoS}_{2}$ single crystals. ${ }^{143}$ Distorted $\mathrm{MoS}_{2}$ structures produced by oxidation of $\mathrm{K}_{x}\left(\mathrm{H}_{2} \mathrm{O}\right)_{y} \mathrm{MoS}_{2}$ strongly depend on the amount of intercalated potassium. ${ }^{146}$ An incomplete oxidation of $\mathrm{K}_{x}\left(\mathrm{H}_{2} \mathrm{O}\right)_{y} \mathrm{MoS}_{2}$ $(x \approx 0.3)$ leads to the formation of $\mathrm{MoS}_{2}$ crystals with the $2 a \times a$ $\left(1 \mathrm{~T}^{\prime}\right)$ structure, whereas the final product of a prolonged (complete) oxidation displays the $a \sqrt{3} \times a \sqrt{3}\left(1 \mathrm{~T}^{\prime \prime \prime}\right)$ superstructure in the $a b$ plane, where, according to STM, Mo atoms are slightly displaced from their ideal positions forming triangles (trimerization). ${ }^{58,146} \mathrm{~A}$ similar $2 a \times a\left(1 \mathrm{~T}^{\prime}\right)$ structure was obtained by oxidation of $\mathrm{K}_{x}\left(\mathrm{H}_{2} \mathrm{O}\right)_{y} \mathrm{MoS}_{2}(x<0.3)^{146}$ implying a composition-dependant difference in the symmetry of these intercalation compounds. Additionally, the strength of the oxidiser is also reported to affect the crystal symmetry of deintercalated metastable $\operatorname{MoS}_{2}$. By using a mild oxidiser, like $\mathrm{I}_{2}$, to treat hydrated $\mathrm{KMoS}_{2}$ crystals, the $1 \mathrm{~T}^{\prime}$ phase $\mathrm{MoS}_{2}$ crystals were synthesised. ${ }^{68}$ While by using strong oxidisers, $\mathrm{K}_{2} \mathrm{Cr}_{2} \mathrm{O}_{7}$ and $\mathrm{Br}_{2}$, it was possible to attain the $1 \mathrm{~T}^{\prime \prime \prime}$ polymorph of $\mathrm{MoS}_{2}{ }^{27,56,146}$ (Fig. 5e). It is also reported that intercalation compounds 
containing other alkali metals, such as sodium and rubidium, can be used as precursors in the synthesis of $1 \mathrm{~T}^{\prime \prime \prime} \mathrm{MoS}_{2}$ single crystals. $^{56}$

Bulk crystals of the $1 \mathrm{~T}^{\prime} \mathrm{MoSe}_{2}$ with lateral size $\sim 100 \mu \mathrm{m}$ have been demonstrated through a similar deintercalation (with $\mathrm{I}_{2}$ solution) of a precursor obtained through a chemical reaction between alkali metal containing molybdenum precursors, such as potassium or sodium molybdate, and elemental selenium annealed in an inert atmosphere in an ampoule ${ }^{69}$ or in an open system in a reducing $\mathrm{H}_{2} / \mathrm{Ar}$ atmosphere ${ }^{68}$ (Fig. 5d). The role of alkali metal cations $(\mathrm{Na}, \mathrm{K})$ is similar to that of lithium in chemical exfoliation process; namely, being electron donors, these cations presumably stabilise the $1 \mathrm{~T}^{\prime}$ phase over the $2 \mathrm{H}$ phase during the solid-state reaction of the precursor formation. To the best of our knowledge, a direct growth of bulk $1 \mathrm{~T}^{\prime} \mathrm{WS}_{2}$ and $1 \mathrm{~T}^{\prime} \mathrm{WSe}_{2}$ crystals has not been reported so far.

High-purity $1 \mathrm{~T}^{\prime}$ monolayers can then be isolated from the parent bulk crystals either mechanically ${ }^{68}$ or via chemical exfoliation in solution. ${ }^{69}$ In the case of chemical exfoliation, an additional lithium intercalation stage of bulk $1 \mathrm{~T}^{\prime}$ crystals is introduced followed by exfoliation in water. With such approach, the $1 \mathrm{~T}^{\prime}$ phase content in the final product reaches $97 \%{ }^{69}$ (Fig. 4e and 5c). A successful exfoliation of $1 \mathrm{~T}^{\prime} \mathrm{MoS}_{2}$ monolayers by gentle sonication and even shaking of the hydrated $\mathrm{Na}_{x} \mathrm{MoS}_{2}$ or LiMoS 2 crystals immersed in water has also been demonstrated ${ }^{69,93,144}$ (Fig. 5b). As shown by C. Guo et al., the alkali metal cations can then be removed from the colloids of $\mathrm{MoS}_{2}$ monolayers by repeated dialysis. ${ }^{144}$ The reported sizes of thus exfoliated $1 \mathrm{~T}^{\prime} \mathrm{MoS}_{2}$ monolayers vary from a few $\mu \mathrm{m}^{144}$ to a few tens of $\mu \mathrm{m}^{69}$ in the case of agitationassisted exfoliation and chemical exfoliation, respectively. Direct exfoliation of bulk $1 \mathrm{~T}^{\prime} \mathrm{TMD}$ crystals can effectively avoid an incomplete phase transition that is typically manifested as co-existing $2 \mathrm{H} / 1 \mathrm{~T} / 1 \mathrm{~T}^{\prime}$ phases in chemically exfoliated material. ${ }^{85}$ Moreover, this approach also allows protecting the in-plane framework of the $1 \mathrm{~T}^{\prime}$ phase in TMD monolayers from fragmentation due to lithium intercalation thus potentially producing $1 \mathrm{~T}^{\prime}$ domains with larger lateral dimensions.

A phase-selective CVD growth of $\mathrm{MoS}_{2}$ monolayers on mica based on using a similar type of precursor - potassium thiomolybdate, $\mathrm{K}_{2} \mathrm{MoS}_{4}$ - has been proposed recently ${ }^{147}$ (Fig. 5a). The authors found out that the crystallisation of the $1 \mathrm{~T}^{\prime}$ phase of $\mathrm{MoS}_{2}$ becomes favourable in potassium-enriched CVD growth due to the stabilisation of the $1 \mathrm{~T}^{\prime}$ phase in the intermediate $\mathrm{K}_{x} \mathrm{MoS}_{2}$ compound. The crystal phase control was achieved by changing the atmosphere from inert to reducing by adding $\mathrm{H}_{2}$ into the carrier gas. The $\mathrm{MoS}_{2}$ flakes produced by decomposition of $\mathrm{K}_{2} \mathrm{MoS}_{4}$ precursor in an inert (Ar) atmosphere display the $2 \mathrm{H}$ phase, whereas high-purity $1 \mathrm{~T}^{\prime} \mathrm{MoS}_{2}$ flakes were produced if $\mathrm{H}_{2}$ gas was introduced. Thus, $1 \mathrm{~T}^{\prime} \mathrm{MoS}_{2}$ monolayers with over $90 \%$ phase purity can be selectively grown on mica in the temperature range $650-750{ }^{\circ} \mathrm{C}$ and $\mathrm{H}_{2}$ concertation of approximately $5-12 \%$ in the carrier gas mixture (Fig. 4). The choice of a suitable substrate may also play an important role since mica may assist in trapping potassium cations produced during the precursor decomposition facilitating the formation of the $1 \mathrm{~T}^{\prime}$ phase. Interestingly, $1 \mathrm{~T}^{\prime} / 2 \mathrm{H}$ heterobilayers could be selectively produced by simply changing pure $\mathrm{Ar}$ to $\mathrm{Ar} / \mathrm{H}_{2}$ mixture during the crystal growth. The authors have also demonstrated that $\mathrm{Cs}_{2} \mathrm{MoS}_{4}$ and $\mathrm{Rb}_{2} \mathrm{MoS}_{4}$ could be suitable for a phase-selective synthesis of $1 \mathrm{~T}^{\prime} \mathrm{MoS}_{2} \cdot{ }^{147}$ Additionally, the growth of $1 \mathrm{~T}^{\prime} \mathrm{WS}_{2}$ under the same conditions was achieved if $\mathrm{K}_{2} \mathrm{WS}_{4}$ was used as an alkali metal containing precursor. ${ }^{147}$

Similarly to vapour deposition approaches, the reported protocols of hydro- or solvothermal syntheses of the metastable $1 \mathrm{~T}\left(1 \mathrm{~T}^{\prime}\right)$ group VI TMD polymorphs utilise a reaction involving ammonium or alkali metal containing precursors. Typically, $1 \mathrm{~T}\left(1 \mathrm{~T}^{\prime}\right) \mathrm{WS}_{2}$ and $\mathrm{MoS}_{2}$ nanostructures are obtained by reacting ammonium containing salts, like $\left(\mathrm{NH}_{4}\right)_{2} \mathrm{WO}_{4},{ }^{148}$ $\left(\mathrm{NH}_{4}\right)_{10} \mathrm{~W}_{12} \mathrm{O}_{41} \cdot \mathrm{H}_{2} \mathrm{O},{ }^{82}$ and $\left(\mathrm{NH}_{4}\right)_{6} \mathrm{Mo}_{7} \mathrm{O}_{24},{ }^{81}$ with the chalcogen precursor, such as thiourea or thioacetamide, or by thermal decomposition of ammonium thiomolybdate ${ }^{149}$ in solution phase (Fig. 6a). Thus produced material exhibits the morphology of ultra-thin nanosheets or nanoribbons composed of nm-sized co-existing $2 \mathrm{H}$ and $1 \mathrm{~T}\left(1 \mathrm{~T}^{\prime}\right)$ single-crystalline domains. The $1 \mathrm{~T}\left(1 \mathrm{~T}^{\prime}\right)$ phase content in these nanostructures reaches $\sim 61.6 \%{ }^{81}$ (Fig. 4 ). The metastable phases are likely to be stabilised by charged precursor residues trapped between the layers of few-layered nanosheets and nanoribbons. Specifically, presence of the intercalated ammonium uniformly distributed across the nanosheets has been repeatedly verified by elemental mapping and XPS analysis. ${ }^{81,82,150}$ Apart from cationic species, it is also assumed that the $1 \mathrm{~T}^{\prime}$ phase in $\mathrm{MoS}_{2}$ produced by a solvothermal reaction from $\left(\mathrm{HN}_{4}\right)_{2} \mathrm{MoS}_{4}$ can be stabilised by intercalated anionic thiomolybdate moieties. ${ }^{149}$ To verify that, the complete removal of salt residues was achieved by prolonged dialysis and the absence of intercalated ammonium in the synthesised material has been demonstrated by XPS analysis. The solvothermal strategy has been further adapted to form arrays of the $1 \mathrm{~T}^{\prime}$-rich $(60 \%)$ nanosheets $\left(\mathrm{MoS}_{2}\right)$ on conductive support (single wall carbon nanotubes) for the application in electrochemical devices. ${ }^{150}$

An interesting chemical approach to produce the $1 \mathrm{~T}$ phase of TMDs that rely on the conversion of non-van der Waals solids, such as MAX and MXene phases, has been reported recently. ${ }^{151,152}$ In this approach, the transition metal atom coordination might be templated by the symmetry of a parent MAX phase. J. Tang et al. have demonstrated that high quality $\mathrm{TiS}_{2}$ sheets can be synthesised by a high temperature chalcogenation of $\mathrm{Ti}_{3} \mathrm{C}_{2} \mathrm{~T}_{x}$ in $\mathrm{H}_{2} \mathrm{~S}$ atmosphere. ${ }^{151}$ This approach has been further extended to produce group IV and VI disulphides and diselenides, and $1 \mathrm{~T}$ phases of $\mathrm{MoS}_{2}$ and $\mathrm{WS}_{2}$, in particular. ${ }^{152}$ Interestingly, the metastable $1 \mathrm{~T}$ phases have only been obtained in the presence of phosphorus vapour in the chalcogen atmosphere, leading to the formation of P-doped $1 \mathrm{~T} / 2 \mathrm{H}$ mixed-phase material. ${ }^{152}$

2.2.3. Reaction kinetics-driven direct synthesis of the metastable phase. Phase-selective synthesis through a fine tuning of the growth conditions is achievable in TMDs with a small energy difference between the thermodynamically stable and metastable polymorphs. $\mathrm{MoTe}_{2}$ displays the difference of only 
a

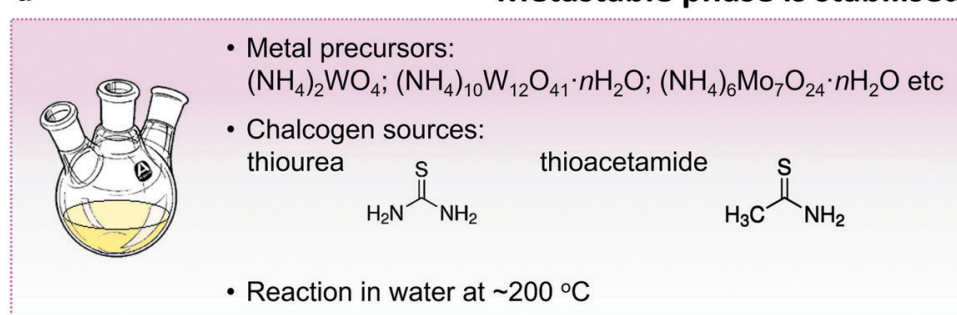

\section{Metastable phase is stabilised by charged precursors' residues}

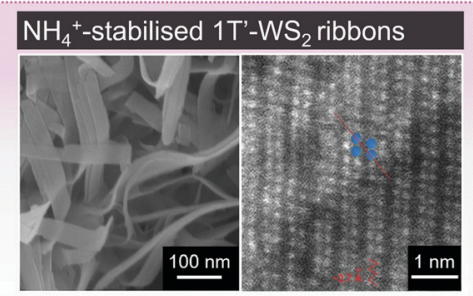

b

Metastable phase is grown due to altering the reaction kinetics

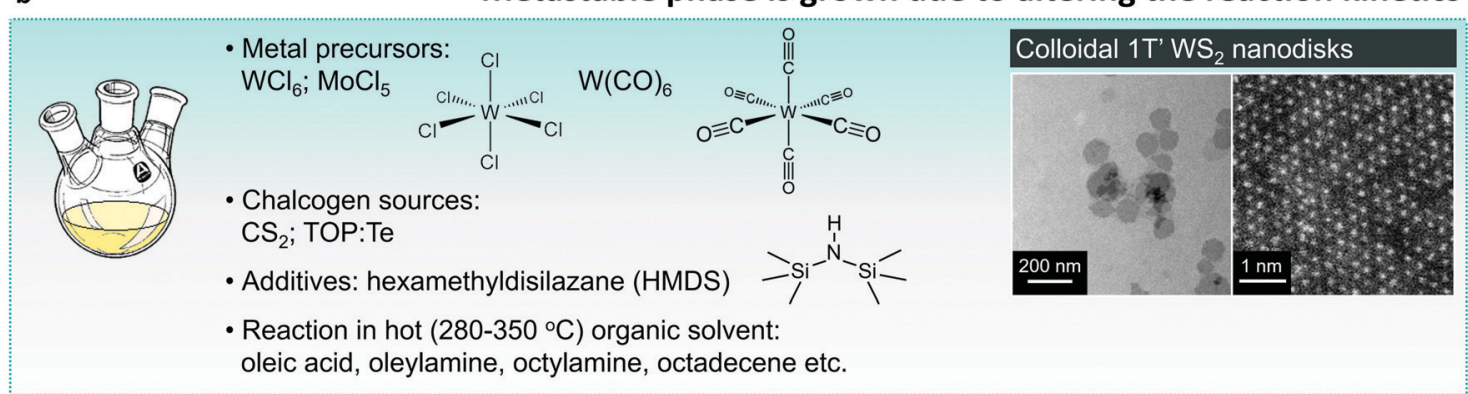

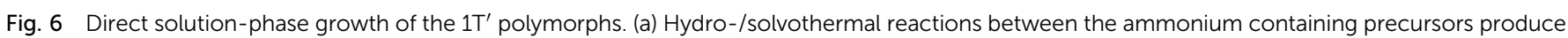

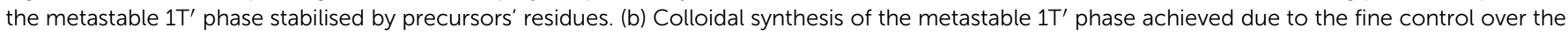

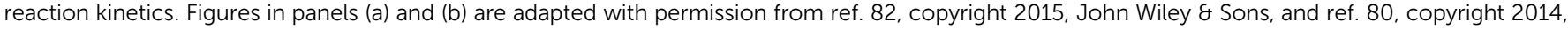
American Chemical Society.

about 35-40 meV per formula unit between the $2 \mathrm{H}$ and $1 \mathrm{~T}^{\prime}$ polymorphs, ${ }^{25,153}$ which is markedly different from the rest of group VI TMDs, and therefore is the most suitable candidate for developing the phase-selective growth strategies. The second candidate could be $\mathrm{WTe}_{2}$ with the difference between stable $1 \mathrm{~T}^{\prime}$ and metastable $2 \mathrm{H}$ being only $90 \mathrm{meV}$ per formula unit. $^{25}$ However, the synthesis of the metastable $2 \mathrm{H} \mathrm{WTe}{ }_{2}$ has not been demonstrated so far.

In vapour phase deposition approaches, the crystal phase tunability has been demonstrated by finely controlling the reaction parameters. For instance, islands of $1 \mathrm{~T}^{\prime} \mathrm{WSe}_{2}$ extended over $\sim 100 \mathrm{~nm}$ have been selectively synthesised on bilayer graphene on $6 \mathrm{H}-\mathrm{SiC}(0001)$ substrates by molecular beam epitaxy (MBE) by keeping the deposition temperature not higher than $280{ }^{\circ} \mathrm{C}^{20,21}$ (Fig. 4). In CVD approach, the crystal phase selectivity is often achieved by controlling the precursor flux. Thus, polycrystalline films of $1 \mathrm{~T}^{\prime} \mathrm{MoTe}_{2}$ with single crystalline domains of $\sim 10 \mathrm{~nm}$ are commonly reported through tellurisation of Mo films or Mo islands in a tellurium-deficient atmosphere. ${ }^{60,154}$ In this case, the formation of the metastable phase is attributed to tellurium deficiency in the produced $\mathrm{MoTe}_{x}$ material that lowers the free energy of $1 \mathrm{~T}^{\prime}$ polymorph. ${ }^{154}$ In particular, Te deficiency greater than 2 at\% suffices the stabilisation of the $1 \mathrm{~T}^{\prime}$ phase over the $2 \mathrm{H}$ in $\mathrm{MoTe}_{2} \cdot{ }^{25,155}$ The lateral dimensions of such phase-uniform polycrystalline $1 \mathrm{~T}^{\prime}$ $\mathrm{MoTe}_{2}$ films are reported to reach a few $\mathrm{cm}^{2} .{ }^{59}$ Interestingly, prolonging the tellurisation time has facilitated a complete solidto-solid $1 \mathrm{~T}^{\prime}$-to- $2 \mathrm{H}$ phase transformation; moreover, the formation of single crystalline $2 \mathrm{H} \mathrm{MoTe} \mathrm{H}_{2}$ domains as large as $2.34 \mathrm{~mm}$ in diameter has been achieved by annealing in a tellurium-enriched atmosphere. ${ }^{154}$ Additionally, the formation of the metastable $1 \mathrm{~T}^{\prime}$ phase by tellurisation of Mo films was attributed to a mechanical strain accumulated between the tellurised and pristine Mo regions. ${ }^{59}$ In such a case, during a prolonged tellurisation, gradual relaxation of the strain triggers structural transformation into the $2 \mathrm{H}$ phase. ${ }^{59}$ It has also been reported that either Mo or $\mathrm{MoO}_{3}$ films can be used as a precursor for the synthesis of few-layered $1 \mathrm{~T}^{\prime} \mathrm{MoTe}_{2}{ }^{156}$ Regardless of the precursor choice, the $1 \mathrm{~T}^{\prime}$ polymorph of $\mathrm{MoTe}_{2}$ was obtained if Te supply was insufficient; however, using $\mathrm{MoO}_{3}$ as precursor led to the formation of morphologically uniform, $\sim 3 \mathrm{~nm}$ thick films (film roughness $\approx 1 \mathrm{~nm}$ ), whereas the roughness of the films produced from Mo precursor under the same conditions was $\approx 5 \mathrm{~nm} .{ }^{156}$ The choice of molybdenum precursor may, however, plays an important role in the CVD synthesis under high tellurium flux. Thus, the same research group have found that $\mathrm{MoO}_{3}$ reacts more easily with Te vapour forming the $2 \mathrm{H}$ phase, while $\mathrm{Mo}$ or $\mathrm{MoO}_{3-x}$ produce the $1 \mathrm{~T}^{\prime}$ phase under the same conditions. ${ }^{157}$ Recently, L. Zhou et al. have demonstrated that planar $1 \mathrm{~T}^{\prime} / 2 \mathrm{H} \mathrm{MoTe}_{2}$ heterostructures can be produced by a one-step tellurisation of $\mathrm{MoO}_{3}$ films using a partially overlapped geometry. ${ }^{158}$ The formation of $2 \mathrm{H}$ phase is attributed to the trapping of Te gas in the gap between the substrate and the cover and therefore to higher concentration of Te residing over the surface of $\mathrm{MoO}_{3}$ film that led to more complete tellurisation; whereas uniform $1 \mathrm{~T}^{\prime}$ phase is obtained in the exposed areas of the substrate. L. Yang et al. have demonstrated that the rate of $\mathrm{MoO}_{3}$ film tellurisation can be controlled by adjusting both the carrier gas flow rate and the reaction temperature; this has allowed the authors to identify the conditions for the selective growth of $1 \mathrm{~T}^{\prime}$ and $2 \mathrm{H} \mathrm{MoTe}_{2}$ fewlayered films. ${ }^{159}$ Alternatively, lateral $1 \mathrm{~T}^{\prime} / 2 \mathrm{H}$ homojunctions can be formed in few-layered $\mathrm{MoTe}_{2}$ films by optimising the precursor 
flux in such a way that the incomplete $1 \mathrm{~T}^{\prime}$-to- $2 \mathrm{H}$ transition is arrested leading to isolated $2 \mathrm{H}$ domains being formed in continuous $1 \mathrm{~T}^{\prime}$ matrix. ${ }^{60,160}$ However attractive as a lever to direct the crystallisation of $\mathrm{MoTe}_{2}$ in the metastable crystal phase, tellurium deficiency of the $1 \mathrm{~T}^{\prime}$ phase produced via metal film tellurisation can significantly hamper its potential use in electronics where high structural perfection is required. ${ }^{161}$ High-quality $1 \mathrm{~T}^{\prime} \mathrm{MoTe}_{2}$ single crystals can be grown directly from elemental Mo and Te by chemical vapour transport (CVT) reaction. ${ }^{58,153,162}$ In this method, a stoichiometric mixture of elemental precursors is sealed in an evacuated ampoule and then maintained at elevated temperature, typically $800-1000{ }^{\circ} \mathrm{C}$, to produce polycrystalline $\mathrm{MoTe}_{2}$ product, which is further recrystallised by CVT method using either $\mathrm{TeCl}_{4},{ }^{63,77} \mathrm{TeBr}_{4},{ }^{162}$ or $\mathrm{I}_{2}{ }^{58}$ as transport agent. The monoclinic $1 \mathrm{~T}^{\prime}$ phase of $\mathrm{MoTe}_{2}$ is thermodynamically stable at the temperatures reported for the CVT processes $\left(900-1100{ }^{\circ} \mathrm{C}\right),{ }^{27,153}$ and can be preserved in the final product if rapidly quenched down to room temperature. ${ }^{58,77}$ The dimensions of thus produced $1 \mathrm{~T}^{\prime}$ $\mathrm{MoTe}_{2}$ single crystals are reported to be as large as $17 \times 10 \times$ $2 \mathrm{~mm}^{3}$ (162) (Fig. 4f). Moreover, D. Keum et al. have shown that stoichiometric $1 \mathrm{~T}^{\prime} \mathrm{MoTe}_{2}$ single crystals can be grown from Mo and Te powders in a molten salt $(\mathrm{NaCl}) .{ }^{153}$ The use of salt was recognised essential since this allows dissolving Te and thus prevents its sublimation and formation of Te deficient product. Similar to ref. $58,1 \mathrm{~T}^{\prime} \mathrm{MoTe}_{2}$ single crystals were attained if the product was quenched, whereas the $2 \mathrm{H}$ phase is a product of slow cooling to room temperature. Atomicallythin $1 \mathrm{~T}^{\prime} \mathrm{MoTe}_{2}$ flakes with the lateral size exceeding $10 \mu \mathrm{m}$ and exhibiting high structural perfection can then be mechanically exfoliated from the bulk crystals onto supports $\left(\mathrm{Si} / \mathrm{SiO}_{2}\right)$ for further examination. ${ }^{58,153}$

By optimising the quenching regime, all three, $1 \mathrm{~T}, 1 \mathrm{~T}^{\prime}$, and $2 \mathrm{H}$, phases of $\mathrm{MoTe}_{2}$ can be selectively produced starting from $\mathrm{MoO}_{3}$ and Te powder precursors. ${ }^{163}$ The fact that all three phases were obtained through the same growth procedure with the only variation being the cooling rate led the authors to conclude that during the direct CVD reaction, $\mathrm{MoTe}_{2}$ nucleates in the $1 \mathrm{~T}$ phase. The $1 \mathrm{~T}$ phase is obtained if after the growth stage the final product is rapidly cooled in the presence of $\mathrm{CO}_{2}$ and $\mathrm{H}_{2}$ gases. Trapping of the metastable $1 \mathrm{~T}$ phase in this case might be caused by the interplay of $\mathrm{CO}_{2}$ and $\mathrm{H}_{2}$ adsorption and interaction with the substrate. If the temperature is reduced more gradually then the $1 \mathrm{~T}^{\prime}$ phase is found to be dominating in the final product. Finally, natural cooling allows enough time for the crystal lattice to transform into the $2 \mathrm{H}$ phase. ${ }^{119}$

A different approach to obtain the metastable phases on monolayered TMDs via CVD has been demonstrated for the synthesis of the $1 \mathrm{~T}$ polymorph of $\mathrm{WS}_{2}{ }^{164}$ and it is based on the use of a catalyst. A mixture of $\mathrm{Fe}_{3} \mathrm{O}_{4}$ and $\mathrm{NaCl}$, serving as a catalyst and a growth promoter, was added into the CVDtubular furnace $\left(\mathrm{WO}_{3}\right.$ and elemental $\mathrm{S}$ as material precursors) leading to the formation of monolayered $1 \mathrm{~T} / 2 \mathrm{H} \mathrm{WS}$ flakes with a butterfly-like shapes, where one of the laterally stitched wings adopted the $1 \mathrm{~T}$ and the other - the $2 \mathrm{H}$ phase. Single crystalline domains in these butterfly-like $\mathrm{WS}_{2}$ monolayers reached $80 \mu \mathrm{m}$ (Fig. 4g). It has also been shown that these $1 \mathrm{~T} / 2 \mathrm{H} \mathrm{WS}_{2}$ structures could serve as seeds for the laterally expanded $1 \mathrm{~T} / 1 \mathrm{~T}$ and $2 \mathrm{H} / 2 \mathrm{H}$ $\mathrm{WS}_{2} / \mathrm{MoS}_{2}$ planar heterostructures. ${ }^{164}$

Owing to the non-equilibrium nature of liquid-phase crystallisation, the reaction pathway in the systems with diverse polymorphism greatly depends on subtle variations in growth conditions and may often lead to the formation of metastable reaction products. ${ }^{165-167}$ First, the crystal growth conditions may be optimised in such a way that the metastable reaction product is kinetically preferred over the equilibrium product. ${ }^{168}$ In fact, it is known that in the systems with numerous polymorphs present, the crystallisation often proceeds through the formation of thermodynamically unstable polymorphs that successively transform into the equilibrium phase; this is known as Ostwald's step rule (Fig. 7b). ${ }^{169}$ The formation of the metastable phase can dominate the crystallisation kinetics owing to a lower nucleation barrier than that of the equilibrium phase $^{170}$ (Fig. 7a) and so one can assume that the metastable polymorph can be isolated if the crystallisation is arrested before the next, more stable, polymorph nucleates and grows. It is assumed that high supersaturations might be prerequisite for such multi-step crystallisation. ${ }^{171,172}$ Also, the free energy of different polymorphs may be altered due to the interaction of the growing crystal with its environment, such as solvent and, especially in the case of colloidal growth, capping ligands, thus lowering the energy barrier for the preferential formation of metastable polymorph and potentially preventing its transformation into the thermodynamically stable one. ${ }^{80}$ In TMD systems, the effects of nanocrystal nucleation and growth kinetics on the preferential formation of one polymorph over the other are still at very early stages of investigation.

Y. Sun et al. have reported that the metastable $1 \mathrm{~T}^{\prime}$ polymorph of $\mathrm{MoTe}_{2}$ is directly accessible in colloidal synthesis. ${ }^{173}$ In their work, flower-like $1 \mathrm{~T}^{\prime} \mathrm{MoTe}_{2}$ nanostructures having diameters of 100-150 $\mathrm{nm}$ were grown in solution phase by continuously injecting molybdenum precursor into a hot mixture $\left(300{ }^{\circ} \mathrm{C}\right)$ containing trioctylphosphine telluride (TOP:Te) in
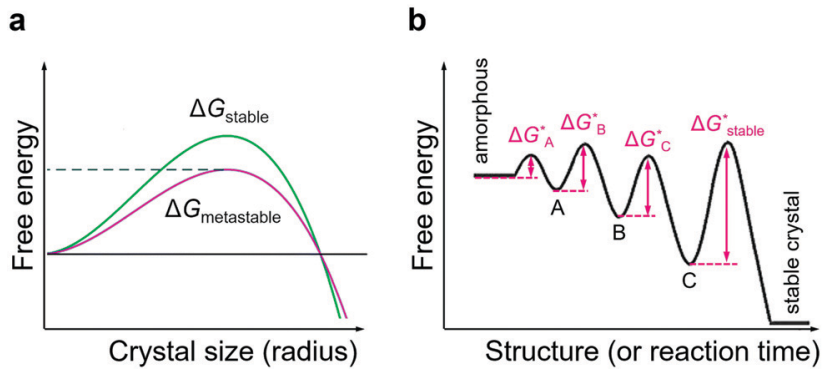

Fig. 7 Homogenous nucleation of the metastable phase in a system with diverse polymorphism. (a) Nucleation barriers of the metastable (purple) and the stable (green) phase in a supersaturated solution. (b) Schematic diagram illustrating the crystallisation pathway of the stable phase proceeding through a series of intermediate metastable phases A, B, and C; $\Delta G^{*}$ denotes the energy barriers of the respective phase transitions. Separate panels are adapted with permission: (a) from ref. 170, copyright 2017, The Royal Society of Chemistry; (b) from ref. [Nature Physics volume 5, pages 68-73 (2009); 10.1038/nphys1148], copyright 2008, Springer Nature. 
trioctylphosphine (TOP) and oleylamine (OlAm). Molybdenum precursor was prepared by dissolving $\mathrm{MoCl}_{5}$ in oleic acid (OlAc). Adding hexamethyldisilazane (HMDS) into tellurium precursor prior to the molybdenum complex injection is required for the formation of crystalline, uniform $\mathrm{MoTe}_{2}$ nanostructures. The authors hypothesised that the interaction with organic ligands can effectively modify the surface energy of $\mathrm{MoTe}_{2}$ nanocrystals and so further lower the energy barrier for forming the metastable structure. Once formed, the metastable $1 \mathrm{~T}^{\prime}$ phase is kinetically trapped due to the pinning at grain boundaries in the petals of $\mathrm{MoTe}_{2}$ nanoflowers. Following a similar synthesis protocol, Y. Sun et al. have obtained highquality $\mathrm{WTe}_{2}$ nanoflowers using $\mathrm{WCl}_{6}$ complex in OlAc as transition metal precursor. ${ }^{174}$ Interestingly, colloidal $\mathrm{WTe}_{2}$ nanoflowers are attained in the orthorhombic Td phase. Additionally, the authors have demonstrated that by using mixtures of $\mathrm{MoCl}_{5}$ and $\mathrm{WCl}_{6}$ as transition metal source, nanostructured $\mathrm{W}_{x} \mathrm{Mo}_{1-x} \mathrm{Te}_{2}$ solid solutions with composition-dependant $\mathrm{Td} / 1 \mathrm{~T}^{\prime}$ crystal phase heterogeneity spanning the $\mathrm{Td} \mathrm{WTe}_{2}$ and $1 \mathrm{~T}^{\prime} \mathrm{MoTe}_{2}$ polymorphs can be accessed. ${ }^{174}$

On the other hand, colloidal synthesis of the metastable polymorphs of group VI disulphides and diselenides remains majorly unexplored. Generally, the metastable polymorphs of these materials are considerably less accessible due to the greater energy barriers of their formation. ${ }^{46}$ Consequently, the solution-phase growth of group VI disulphides and diselenides, whether by a thermolytic decomposition of a single source precursor, like $\left[\mathrm{Mo}_{2} \mathrm{O}_{2} \mathrm{~S}_{2}\left(\mathrm{~S}_{2} \mathrm{COEt}\right)_{2}\right],{ }^{175}$ or by a direct reaction between molecular precursors, such as $\mathrm{MoCl}_{5}$ and $\mathrm{CS}_{2},{ }^{176}$ commonly results in the thermodynamically stable $2 \mathrm{H}$ polymorph. To date, the reported protocols include the use of tungsten and molybdenum hexacarbonyls ${ }^{177}$ and chlorides, ${ }^{178-180}$ molybdenyl acetylacetonate, ${ }^{181}$ sodium molybdate ${ }^{179,182}$ and sodium tungstate, ${ }^{183}$ as a source of transition metal. Chalcogen precursors include carbon disulphide,${ }^{176,180}$ diphenyl diselenide; ${ }^{177,180,181}$ complexes of elemental sulphur ${ }^{178}$ or selenium ${ }^{179,182,183}$ in organic solvents, like oleylamine or octadecene. Despite such a variety of precursors available for a direct solution-phase growth of group VI TMDs, only the semiconducting $2 \mathrm{H}$ phase was synthesised. However, a slow sulphidation of colloidal $\mathrm{W}_{18} \mathrm{O}_{49}$ nanorods, serving as sacrificial seeds, by in situ generated $\mathrm{H}_{2} \mathrm{~S}$ has led to the formation of irregularly-shaped ultrathin $\mathrm{WS}_{2}$ disks demonstrating co-existing $2 \mathrm{H}$ and $1 \mathrm{~T}^{\prime}$ crystal phases. ${ }^{184}$

B. Mahler et al. have reported on a phase-selective colloidal synthesis of $\mathrm{WS}_{2}$ nanosheets ${ }^{80}$ (Fig. 6b). Disk-like $\mathrm{WS}_{2}$ nanostructures with the distorted octahedral $1 \mathrm{~T}^{\prime}$ crystal structure were synthesised through a reaction between $\mathrm{WCl}_{6}$ complex in oleic acid and $\mathrm{CS}_{2}$ in hot $\left(320^{\circ} \mathrm{C}\right)$ oleylamine, whereas the $2 \mathrm{H}$ $\mathrm{WS}_{2}$ nanosheets were grown from the same molecular precursors in the presence of an additive, hexamethyldisilazane (HMDS). HMDS is an essential chemical in colloidal synthesis of another class of layered materials: narrow-gap $\mathrm{A}^{\mathrm{IV}}-\mathrm{B}^{\mathrm{VI}}$ semiconductors, such as GeS and SnS. ${ }^{185,186}$ It has been noted that the growth from the respective transition metal halides in hot oleylamine in the absence of HMDS leads to amorphous product, whereas in the presence of HMDS, highly-uniform, single crystalline plates of SnS and GeS are being formed. ${ }^{185-187}$ In the case group VI TMDs, Y. Sun et al. have reported that the presence of HMDS is required for the formation of highly crystalline $\mathrm{MoTe}_{2},{ }^{173} \mathrm{WTe}_{2}{ }^{174}$ and $\mathrm{W}_{x} \mathrm{Mo}_{1-x} \mathrm{Te}_{2}{ }^{174}$ colloidal nanosheets. M. Kobylinski et al. have suggested that in general, the role of HMDS in colloidal synthesis from transition metal chlorides is two-fold: first, HMDS may react with the transition metal source, forming the corresponding (trimethylsilyl)amino complex; second, HMDS may bind chloride ions and remove them from the reaction mixture as (trimethylsilyl) chloride. While B. Mahler et al. hypothesised that in the specific case of colloidal synthesis of $\mathrm{WS}_{2}$, HMDS may effectively tune the reactivity of tungsten precursor enabling the phase-selective growth. ${ }^{80}$ Specifically, they presume that HMDS may partially substitute the oleate ligands complexing tungsten ions, leading to the formation of a more active form of tungsten precursor. Their alternative hypothesis suggests that HMDS may act as a capping ligand during the nanocrystal growth, stabilising the $2 \mathrm{H}$ polymorph over the $1 \mathrm{~T}^{\prime}$. In order to verify which of the two possible mechanism is more likely to drive the reaction, the authors replace oleic acid (OlAc) with another strongly binding ligand - dodecanethiol (DDT). The authors suggest that unlike OlAc, DDT does not react with HMDS thus the formation of an activated tungsten (trimethylsilyl)amino complex is not possible in the DDT protocol. Indeed, the material grown from $\mathrm{WCl}_{6}$-DDT complex in the presence of HMDS exhibits the $1 \mathrm{~T}^{\prime}$ phase. These findings suggest that the formation of the thermodynamically stable $2 \mathrm{H}$ phase of $\mathrm{WS}_{2}$ can be mainly attributed to the activation of tungsten complex by reacting with HMDS, whereas less reactive tungsten oleate may lead to the direct growth of the $1 \mathrm{~T}^{\prime}$ phase. It should be noted that a detailed study of the suggested intermediates in the $\mathrm{WCl}_{6}-\mathrm{OlAc}$ and $\mathrm{WCl}_{6}-\mathrm{DDT}$ systems, their activity and possible reactions with HMDS is not provided in the cited work. ${ }^{80}$

In addition to $1 \mathrm{~T}^{\prime} \mathrm{WS}_{2}$, the direct growth of the metastable $1 \mathrm{~T}^{\prime}$ polymorph of $\mathrm{WSe}_{2}$ has been reported recently ${ }^{71,188}$ (Fig. 4d). It is worth mentioning that the $1 \mathrm{~T}^{\prime}$ polymorphs of $\mathrm{WSe}_{2}$ and $\mathrm{MoSe}_{2}$ were not previously demonstrated by $\mathrm{Li}$ intercalation and were only achieved via direct synthesis. ${ }^{68,71}$ Uniform $1 \mathrm{~T}^{\prime} \mathrm{WSe}_{2}$ nanoflowers with an average diameter of $200 \mathrm{~nm}$ and composed of highly-crystalline atomically-thin petals were grown through a reaction between $\mathrm{W}(\mathrm{CO})_{6}$ and trioctylphosphine selenide (TOP:Se) in hot oleic acid $\left(300{ }^{\circ} \mathrm{C}\right) .{ }^{71}$ Prior to that work, only a few experimental protocols on colloidal synthesis of $\mathrm{WSe}_{2}$ nanostructures were present, but only the formation of thermodynamically stable $2 \mathrm{H}$ polymorph was reported. ${ }^{177,180}$ In order to elucidate the reaction pathway, M. Sokolikova et al. have performed a parametric study of growth conditions. The activity of both precursors was systematically varied by replacing $\mathrm{W}(\mathrm{CO})_{6}$ with $\mathrm{WCl}_{6}$, TOP:Se with elemental Se; additionally, the activity of tungsten precursor was modified by complexing with two additives: hexamethyldisilazane (HMDS) and tetradecylphosphonic acid (TDPA). Interestingly, the metastable $1 \mathrm{~T}^{\prime}$ crystal phase was consistently grown under these conditions; only negligible changes in the 
morphology were reported. Based on these observations, the authors have proposed that under the described experimental conditions, the formation of metastable $1 \mathrm{~T}^{\prime}$ polymorph might be attributed to the crystallisation in kinetically-driven regime; however, switching between the supposedly kineticallydriven and thermodynamically-controlled regimes has not been unambiguously demonstrated.

Later, J. Geisenhoff et al. have demonstrated a phaseselective synthesis of $\mathrm{WSe}_{2}$ nanoflowers that was achieved in a biligand system of trioctylphosphine oxide (TOPO) and oleic acid (OlAc). ${ }^{188}$ The metastable $1 \mathrm{~T}^{\prime}$ phase of $\mathrm{WSe}_{2}$ was obtained when the fraction of OlAc in the ligand mixture was high, while keeping the oleic acid fraction to minimum has led to the growth of the thermodynamically stable $2 \mathrm{H}$ phase. The authors indicate that by coordinating with oleic acid, a less reactive form of tungsten precursor is formed; this leads to nucleation of fewer seeds and to larger nanocrystals compared to the synthesis in TOPO-rich mixtures. However, the effect of ligand mixture composition on the preferential nucleation of one of the other crystal phases remains unclear. Regardless of the ligand ratio, colloidal $\mathrm{WSe}_{2}$ was found to nucleate in the metastable $1 \mathrm{~T}^{\prime}$ phase; however, in the systems, containing low fraction of OlAc, it converts into the $2 \mathrm{H}$ phase over time. ${ }^{188}$ Whereas in the case of ligand mixtures with high OlAc content or of syntheses in OlAc, the final product seemingly retains the metastable $1 \mathrm{~T}^{\prime}$ phase. $^{188}$

Recently, it has been proposed that the energy barrier for the homogeneous nucleation of the metastable $1 \mathrm{~T}$ phase could be lowered in a magneto-hydrothermal process. ${ }^{136}$ The preferential nucleation of the $1 \mathrm{~T}$ polymorph in high magnetic fields is facilitated by the higher magnetic susceptibility of the $1 \mathrm{~T}$ structure, compared to the $2 \mathrm{H}$ phase. Using this approach, W. Ding et al. have selectively synthesised $1 \mathrm{~T} \mathrm{MoS}_{2}$ and $\mathrm{WS}_{2}$ nanosheets through a hydrothermal reaction between either ammonium heptamolybdate $\left(\left(\mathrm{NH}_{4}\right)_{6} \mathrm{Mo}_{7} \mathrm{O}_{24} \cdot 4 \mathrm{H}_{2} \mathrm{O}\right)$ and thiourea or tungsten hexachloride $\left(\mathrm{WCl}_{6}\right)$, and thioacetamide under an external magnetic field of $\sim 9 \mathrm{~T}^{136}$ The nucleation of the $2 \mathrm{H}$ phase from the same precursors in the absence of magnetic field (control) has also been demonstrated.

Considering the present evidence, we suggest that the understanding of crystallisation pathways leading to the formation of metastable polymorphs of group VI TMDs in colloidal solution is at early stages. A thorough investigation of the nucleation mechanism, including the nature of reaction intermediates formed from molecular precursors, possible prenucleation clusters, energetics of the nanocrystal-solution interface, and surface dynamics of capping ligands on the nanocrystal facets, is needed in order to gain control over the phase-selective growth of various polymorphs of group VI TMDs.

It is interesting to note that unlike other van der Waals solids, such as colloidal $\mathrm{SnS}^{186}$ and $\mathrm{SnSe},{ }^{189} \mathrm{GeS}$ and GeSe, ${ }^{185}$ InSe, ${ }^{190}$ and group IV $^{191,192}$ and $\mathrm{V}^{192}$ disulphides and diselenides, that are acquired in a form of two-dimensional platelets and disks, group VI TMDs obtained through a solution-phase (hydro-/solvothermal ${ }^{81,193}$ and colloidal ${ }^{71,173,174,179,180}$ ) reaction between similar type of precursors typically exhibit branched morphology of flower-like structures composed of ultra-thin petals. The overall anisotropic morphology of van der Waals compounds is expected since the lateral extension in the $a b$ plane is energetically preferred due to surface energy difference between the undercoordinated edge facets and the basal planes, where dangling bonds are absent. ${ }^{192,194}$ In some cases, like layered $\mathrm{SnSe}^{189}$ and $\mathrm{Bi}_{2} \mathrm{Te}_{3},{ }^{195}$ and non-layered $\mathrm{PbS}^{196}$ systems, the growth of large regularly-shaped single-crystalline nanostructures does not proceed through the extension of primary nucleated seeds but rather occurs via the two-dimensional oriented attachment of the seeds. The self-assembly process is driven by the minimisation of surface energy associated with the unsaturated bonds, for example, in the lateral surface of SnSe seeds, and can be effectively controlled by varying the precursors' concentrations. ${ }^{189}$ However, neither of these crystallisation mechanisms can explain the formation of branched flower-like structures of group VI TMDs produced via a solution-phase reaction. The formation of $3 \mathrm{D}$ flower-like nanostructures composed of individual 2D nanosheets might be caused by a nucleation from a dense amorphous state formed in the supersaturated solution as proposed for $\mathrm{TiS}_{2}$ nanoflowers. ${ }^{197} \mathrm{~S}$. Prabakar et al. have found out that $\mathrm{TiS}_{2}$ flower-like structures evolve in colloidal solution starting with the formation of amorphous globules and proceeding through the appearance of randomly oriented crystalline platelets on the surface of amorphous globules to the growth of these petals originating from the central core so that the amorphous core is no longer apparent. ${ }^{197}$ Similar formation mechanism was later described by O. Meiron et al. ${ }^{198}$ and by C. Zhang et $a .^{182}$ for colloidal $\mathrm{MoS}_{2 x} \mathrm{Se}_{2-2 x}$ and $\mathrm{MoSe}_{2}$ nanoflowers, respectively. $\mathrm{O}$ Meiron et al. have identified amorphous blobs that are formed at the very early stages of the reaction; these later serve as material reservoirs for the curled and tangled nanosheets that crystallise inside the amorphous clusters. ${ }^{198}$ Over time, amorphous material is fully consumed and uniform nanoflowers are formed. Despite a considerable progress in controlled synthesis of TMDs, we highlight that a deeper understanding of solution-phase nucleation and crystallisation processes of group VI TMDs is required in order to fully employ their potential in practical applications.

\section{Comparison of various techniques to access the metastable $1 \mathrm{~T}\left(\mathbf{1 T}^{\prime}\right)$ phases}

Fig. 4 provides a comprehensive overview of the reported approaches to access the metastable $1 \mathrm{~T}\left(1 \mathrm{~T}^{\prime}\right)$ phases of group VI TMDs. The data points were taken from the references, where the lateral dimensions of continuous domains (not necessarily single crystalline) of the metastable $1 \mathrm{~T}\left(1 \mathrm{~T}^{\prime}\right)$ phase were clearly stated and the metastable phase content was determined from the results of either XPS or Raman spectroscopy. These are listed in Table 1.

The most commonly reported approach to synthesise the metastable phase in measurable quantities is chemical exfoliation from bulk TMD powders. In chemically exfoliated $\mathrm{MoS}_{2}$ and $\mathrm{WS}_{2}$, the $1 \mathrm{~T}\left(1 \mathrm{~T}^{\prime}\right)$ phase is found as nm-scale domains embedded in the $2 \mathrm{H}$ matrix, although the total $1 \mathrm{~T}\left(1 \mathrm{~T}^{\prime}\right)$ phase content reaches $\sim 70-80 \%$. It should be noted that there is little evidence that this approach is effective in the case of group VI diselenides. 
Table 1 Comparison of various synthesis techniques to access the metastable $1 \mathrm{~T}\left(1 \mathrm{~T}^{\prime}\right)$ phases of group VI TMDs

\begin{tabular}{llll}
\hline Material & Lateral size & \% of $1 \mathrm{~T}\left(1 \mathrm{~T}^{\prime}\right)$ & Ref. \\
\hline $\mathrm{Molecular}$ beam epitaxy & $4 \mathrm{~nm}$ & 100 & \\
$\mathrm{MoS}_{2}$ & $10 \mathrm{~nm}$ & 100 & 79 \\
$\mathrm{MoSe}_{2}$ & $100 \mathrm{~nm}$ & 100 & 70 \\
$\mathrm{WSe}_{2}$ & & & 21
\end{tabular}

Chemical vapour transport

$\begin{array}{lll}\mathrm{MoS}_{2} & 100 \mu \mathrm{m} & 100\end{array}$

$150 \mu \mathrm{m} \quad 100$

$500 \mu \mathrm{m} \quad 100$

$\mathrm{MoSe}_{2} \quad 170 \mu \mathrm{m} \quad 100$

$\begin{array}{lll}\mathrm{MoTe}_{2} & 10 \mu \mathrm{m} & 100\end{array}$

$1 \mathrm{~cm} \quad 100$

$1.7 \mathrm{~cm} \quad 100$

Ref.

21

1

$1.7 \mathrm{~cm}$

$\begin{array}{lccc}\text { Chemical vapour deposition } & & \\ \mathrm{MoS}_{2} & 10 \mu \mathrm{m} & 100 & 147 \\ \mathrm{WS}_{2} & 80 \mu \mathrm{m} & 100 & 164\end{array}$

Chemical exfoliation (both $2 \mathrm{H}$ and $1 \mathrm{~T}^{\prime}$ crystals)

$\begin{array}{llll}\mathrm{MoS}_{2} & 3.5 \mathrm{~nm} & 70 & 112\end{array}$

$\begin{array}{llll}\mathrm{WS}_{2} & 3 \mathrm{~nm} & 80 & 112\end{array}$

$\begin{array}{llll} & 100 \mathrm{~nm} & 80 & 83 \\ \mathrm{MoS}_{2} & 1 \mu \mathrm{m} & 70 & 101\end{array}$

$\begin{array}{lll}700 \mathrm{~nm} & 80 & 103\end{array}$

$\begin{array}{lll}10 \mu \mathrm{m} & 97 & 69\end{array}$

$\begin{array}{lrr}60 \mu \mathrm{m} & 97 & 69\end{array}$

$500 \mathrm{~nm} \quad 100 \quad 93$

$1.5 \mu \mathrm{m} \quad 100 \quad 265$

Direct colloidal/hydrothermal synthesis

$\begin{array}{llll}\mathrm{MoS}_{2} & 200 \mathrm{~nm} & 61.6 & 81 \\ \mathrm{WS}_{2} & 100 \mathrm{~nm} & 50 & 80 \\ & 162 \mathrm{~nm} & 66.4 & 148 \\ \mathrm{WSe}_{2} & 100 \mathrm{~nm} & 60 & 71\end{array}$

On the other hand, CVT growth, that up until recently was employed to synthesise cm-sized single crystals of $1 \mathrm{~T}^{\prime} \mathrm{MoTe}_{2}$ only, has been extended to the case of molybdenum disulphide and diselenide. The growth of the metastable phase is achieved with the assistance of alkali metal containing precursors and $1 \mathrm{~T}^{\prime} \mathrm{MoS}_{2}$ and $1 \mathrm{~T}^{\prime} \mathrm{MoSe}_{2}$ crystals with lateral dimensions of a few hundreds of $\mu \mathrm{m}$ are produced by gentle deintercalation of the intermediate products. Furthermore, chemical exfoliation from bulk $1 \mathrm{~T}^{\prime} \mathrm{MoS}_{2}$ and $1 \mathrm{~T} \mathrm{MoSe}_{2}$ crystals results in unprecedentedly large nanosheets with the metastable phase content of nearly $100 \%$. CVD growth, that typically leads to the formation of the thermodynamically stable polymorph, can be optimised in such a way that the metastable phase is selectively produced. Thus, in the presence of a catalyst or alkali metal containing precursors, the growth of single crystalline domains of $1 \mathrm{~T} \mathrm{WS}_{2}$ and $1 \mathrm{~T}^{\prime} \mathrm{MoS}_{2}$ with the sizes of a few tens of $\mu \mathrm{m}$ has been reported. Another deposition approach, molecular beam epitaxy, allows for the growth of islands of $1 \mathrm{~T}^{\prime}$ phase with lateral dimensions varying from a few nm as in the case of $\mathrm{MoS}_{2}$ and $\mathrm{MoSe}_{2}$ to nearly reaching $100 \mathrm{~nm}$ in the case of $\mathrm{WSe}_{2}$. It should be noted that the selectivity of MBE process is rather low at present as the formation of separate domains of the $2 \mathrm{H}$ phase is often reported.

Solution-phase approaches, such as hydrothermal and colloidal reactions, allow for the synthesis of measurable quantities of the $1 \mathrm{~T}^{\prime}$ phase containing material, in some cases reaching a few grams. The lateral size of $1 \mathrm{~T}^{\prime}$ domains are reported within the range from a few tens to a few hundreds of $\mathrm{nm}$, and the metastable phase content is about $50-60 \%$.

\section{How stable the metastable $1 \mathrm{~T}\left(\mathbf{1 T ^ { \prime }}\right)$ phases are?}

Although being thermodynamically metastable under ambient conditions, the $1 \mathrm{~T}\left(1 \mathrm{~T}^{\prime}\right)$ polymorph is kinetically stable over the course of a few days to a few months, depending on the material morphology and environment. Thus, chemically exfoliated $1 \mathrm{~T}^{\prime}$ and $1 \mathrm{~T}^{\prime \prime \prime} \mathrm{MoS}_{2}$ monolayers gradually convert back to the $2 \mathrm{H}$ phase over 3-5 days in air as demonstrated by Raman ${ }^{87}$ and STM $^{146}$ studies. The conversion is attributed to the restacking of exfoliated material in suspension that leads to the van der Waals interactions between the layers being recovered. The transformation into the $2 \mathrm{H}$ phase is significantly promoted when the exfoliated $1 \mathrm{~T}^{\prime}$ TMDs are deposited to form thin films owing to the removal of water from the interlayer space of exfoliated sheets during drying. ${ }^{87}$ Synthesised through a direct hydrothermal reaction, the 1T-phase $\mathrm{MoS}_{2}$ was reported to slowly convert into the $2 \mathrm{H}$ phase over 90 days in water. This was accompanied by a gradual loss of the catalytic activity. ${ }^{199}$

\section{Property-related applications of the metastable $1 \mathrm{~T}\left(\mathbf{1 T}^{\prime}\right)$ phases}

The metastable $1 \mathrm{~T}\left(1 \mathrm{~T}^{\prime}\right)$ polymorphs of group VI TMDs are found to be promising for various applications, including electrocatalysis, ${ }^{83,103}$ electrochemical energy storage, ${ }^{101,200}$ sensors, ${ }^{201,202}$ transparent electrodes for nanoscaled optoelectronics ${ }^{110}$ and photovoltaics, ${ }^{203}$ transport layers for solar cells, ${ }^{204}$ and even microwave absorbers for application in electromagnetic shielding and stealth camouflage. ${ }^{205}$ The incorporation of domains of the $1 \mathrm{~T}$ phase in the $2 \mathrm{H}$ matrix induces a robust ferromagnetism in the intrinsically nonmagnetic semiconducting TMDs, further expanding the range of potential applications to spintronic devices. ${ }^{206,207}$ The difficulty in accessing the input of the metastable phase is commonly associated with the fact that in many cases, the $1 \mathrm{~T}\left(1 \mathrm{~T}^{\prime}\right)$ phase co-exists with the thermodynamically stable $2 \mathrm{H}$ phase in these produced materials. Here, we review the state-of-the-art research articles related to the understanding of how the intrinsic properties of the $1 \mathrm{~T}\left(1 \mathrm{~T}^{\prime}\right)$ phases can enable novel applications.

5.1. Basal plane activation for efficient electrocatalysis. Group VI TMDs have been actively studied as catalysts for the hydrogen evolution reaction (HER). ${ }^{83,115,208-215}$ Electrocatalytic hydrogen generation is practically achieved by water electrolysis, typically performed in acidic medium. The initial reduction of solvated protons by electrons from the external circuit leads to the formation of adsorbed neutral hydrogen atom on the catalyst surface; this step is known as the Volmer reaction. Then, the formation of molecular hydrogen proceeds through either the Tafel reaction or the Heyrovsky reaction. The former one involves a reaction between two adsorbed surface hydrogen atoms. In the latter, the adsorbed hydrogen reacts with the solvated proton followed by reduction by electrons from the external circuit. ${ }^{216}$ Depending on the electrocatalyst nature, the rate limiting step of the HER can be either the initial proton reduction or the following formation of molecular hydrogen. 
Electrocatalysis: Hydrogen evolution
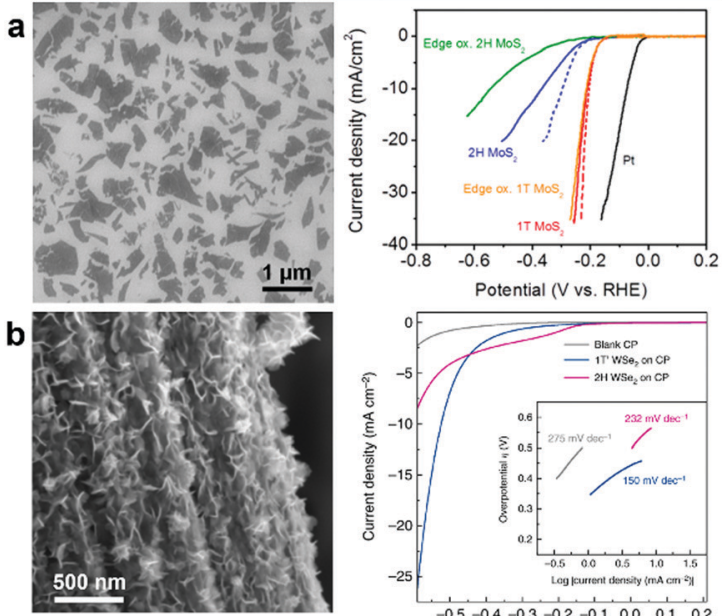

Potential (V vs. RHE)

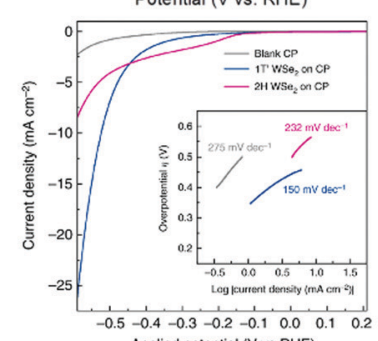

Apolied potential ( $V$ vs RHE)

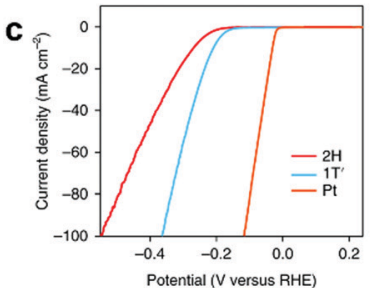

Supercapacitors

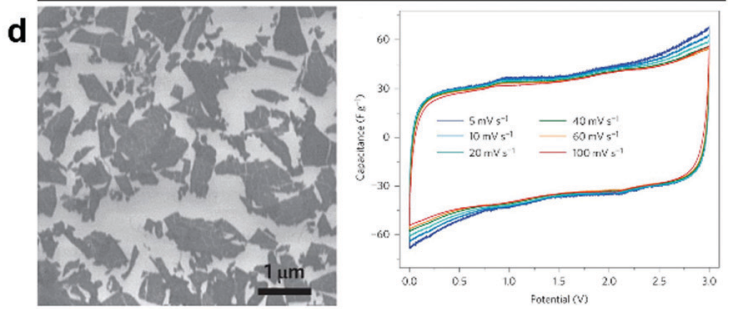

\section{Li-ion batteries}

e
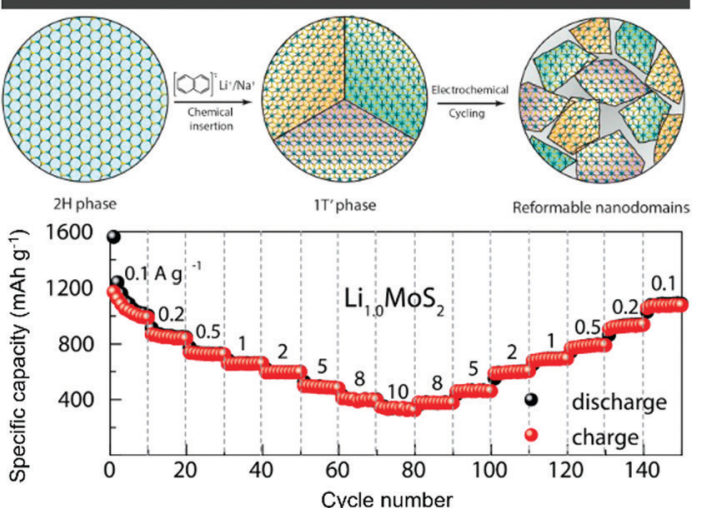

Fig. 8 Applications of the metastable $1 T\left(1 T^{\prime}\right)$ phases of group VI TMDs in electrochemical energy storage and conversion. (a) SEM image (left) of micronsized chemically exfoliated $1 \mathrm{~T}^{\prime} \mathrm{MoS}_{2}$ nanosheets and the polarisation curves (right) of $1 \mathrm{~T}^{\prime}$ and $2 \mathrm{H} \mathrm{MoS}_{2}$ demonstrating the basal plane activation in the $1 \mathrm{~T}^{\prime}$ polymorph. (b) SEM image (left) of $1 T^{\prime}$ WS $e_{2}$ nanosheets grown directly on carbon fibres demonstrating lower HER overpotentials and lower Tafel slopes (right) compared to the corresponding $2 \mathrm{H}$ phase. (c) Polarisation curves (left) and the corresponding Tafel slopes (right) of the CVD grown $1 \mathrm{~T}^{\prime} \mathrm{MoS}_{2}$ flakes. (d) SEM image (left) of micron-sized chemically exfoliated $\mathrm{MoS}_{2}$ nanosheets and CV curves (right) of a symmetric supercapacitor device in an organic electrolyte acquired at various scan rates. (e) A schematic (top) illustrating the domain size reduction during a continuous cycling of lithiated $\mathrm{MoS}_{2}$ nanosheets and charge-discharge plots (bottom) at different rates for the lithiated $\mathrm{MoS}_{2}$-based batteries. Separate panels are adapted with permission: (a) from ref. 103, copyright 2013, American Chemical Society; (b) from ref. 71, copyright 2019, Springer Nature; (c) from ref. 147, copyright 2018, Springer Nature; (d) from ref. 101, copyright 2015, Springer Nature; and (e) from ref. 200, copyright 2016, American Chemical Society.

The widely accepted descriptor for the HER rate is the Gibbs free energy $\Delta G_{\mathrm{H}}$ which characterises the adsorption of hydrogen on the active site. ${ }^{217} \mathrm{~A}$ good catalyst for the HER binds hydrogen neither weakly nor strongly $\left(\Delta G_{\mathrm{H}}\right.$ is close to 0$)$. First-principles calculations have identified $\mathrm{MoS}_{2}$ as a novel type of catalyst with a potential to replace Pt-group materials in electrocatalytic hydrogen production. ${ }^{218}$ Although bulk $\mathrm{MoS}_{2}$ is a poor catalyst, the catalytic activity of nanostructured $\mathrm{MoS}_{2}$ is attributed to the distinct metallic states localised at the Mo-terminated $(10-10)$ edges. ${ }^{17}$ These one-dimensional states were first predicted by M. Bollinger et al. ${ }^{219}$ and later visualised using scanning tunnelling microscopy ${ }^{219}$ and scanning transmission X-ray microscopy. ${ }^{220}$ The thermochemistry predictions were verified by T. Jaramillo et al. by measuring the catalytic activity of UHV sputtered $\mathrm{MoS}_{2}$ nanoislands of various sizes. ${ }^{17}$ The observed correlation between the rate of hydrogen evolution and the fraction of edge sites of $\mathrm{MoS}_{2}$ nanoparticles unambiguously suggests that in the case of semiconducting $2 \mathrm{H} \mathrm{MoS}_{2}$ nanostructures, the electrocatalytic HER predominantly takes place at the edge sites. ${ }^{17}$ Among $2 \mathrm{H}$ group VI TMDs, $\mathrm{MoSe}_{2}$ is expected to demonstrate the highest catalytic activity since both $(10-10)$ metal- and $(-1010)$ chalcogen-terminated edges have $\Delta G_{\mathrm{H}}$ close to thermoneutral
(0.02 and $-0.05 \mathrm{eV}$, respectively). ${ }^{221} \mathrm{MoSe}_{2}$ is closely followed by $\mathrm{WS}_{2}$, both edges of which are potentially active for the catalytic HER $(-0.04$ and $-0.05 \mathrm{eV})$, whereas only the Se edge of $\mathrm{WSe}_{2}$ $(-0.05 \mathrm{eV})$ and the Mo edge of $\mathrm{MoS}_{2}(0.06 \mathrm{eV})$ were identified as catalytically active. ${ }^{221}$ The basal planes of $2 \mathrm{H}$ group VI TMDs are found to be catalytically inert with $\Delta G_{\mathrm{H}}$ close to $2 \mathrm{eV}^{221}$ Theoretically, higher affinity of basal planes of metallic $1 \mathrm{~T}\left(1 \mathrm{~T}^{\prime}\right)$ TMDs to hydrogen can lead to a remarkable improvement of their catalytic performance as compared to the semiconducting $2 \mathrm{H}$ counterparts. ${ }^{18,221,222}$ Experimentally, the activation of the basal plane of the metallic 1T phase has been demonstrated by partially oxidising chemically exfoliated $\mathrm{MoS}_{2}$ nanosheets. ${ }^{103} \mathrm{D}$. Voiry et al. have compared the performance of $1 \mathrm{~T}$ and $2 \mathrm{H} \mathrm{MoS}_{2}$ nanosheets before and after oxidative treatment. They reported that the catalytic activity of edge oxidised $2 \mathrm{H} \mathrm{MoS}_{2}$ nanosheets decreased considerably, while the performance of $1 \mathrm{~T}$ phase remained unaffected. The activation of basal planes and so the higher density of catalytically active sites in metallic polymorphs is considered as one of the reasons why the $1 \mathrm{~T}\left(1 \mathrm{~T}^{\prime}\right)$ polymorphs outperform their $2 \mathrm{H}$ counterparts in catalytic HER. ${ }^{83,103,223}$ This activity enhancement has been demonstrated for both group VI TMD nanosheets ${ }^{103}$ and quantum dots ${ }^{112}$ (Fig. 8a). 
The enhanced catalytic activity of the metastable polymorphs of group VI TMDs is also associated with higher conductivity of the metallic (semimetallic) $1 \mathrm{~T}\left(1 \mathrm{~T}^{\prime}\right)$ phases. Recently, it has been suggested that the characteristics of the interface between active material and functional substrate, as well as the electron transport across active material, affect the catalytic performance even more than the thermodynamics of catalytic reaction. Compared to the semiconducting $2 \mathrm{H}$ counterparts, the $1 \mathrm{~T}\left(1 \mathrm{~T}^{\prime}\right)$ phases of $\mathrm{WS}_{2}$, $\mathrm{MoS}_{2}$ and $\mathrm{MoSe}_{2}$ greatly benefit from faster charge transfer in metallic polymorphs. ${ }^{102,199,224}$ Additionally, in the case of metallic polymorphs, the catalyst-substrate interface becomes essentially metal-metal, which leads to more effective electron injection into the active material. ${ }^{225}$ It is worth mentioning that the $1 \mathrm{~T}^{\prime}$ phase of $\mathrm{WSe}_{2}$ have shown the enhanced electrocatalytic activity compared to its $2 \mathrm{H}$ counterpart (Fig. 8b); however, in this case the material was obtained uniquely via direct synthesis. ${ }^{71}$

The catalytic activity of metastable $1 \mathrm{~T}$ and $1 \mathrm{~T}^{\prime}$ polymorphs of group VI TMDs cannot be compared directly as both phases are often reported interchangeably. ${ }^{83}$ Further, L. Wang et al. have suggested that during electrochemical cycling, the 1T phase of $\mathrm{MoS}_{2}$ irreversibly transforms into the more catalytically active $1 \mathrm{~T}^{\prime}$ phase. ${ }^{226}$ According to first principle calculations, the 1T phase readily transforms into the $1 \mathrm{~T}^{\prime}$ phase at room temperature and this transition is considerably promoted by hydrogen adsorption during the $\mathrm{H}_{2}$ evolution. Moreover, the authors have experimentally proved that the $1 \mathrm{~T}-$ to- $1 \mathrm{~T}^{\prime}$ transition does occur in chemically exfoliated $\mathrm{MoS}_{2}$ and they suggest that such a transition may explain the self-optimising catalytic behaviour reported for the group VI TMD catalysts. ${ }^{226}$ Additionally, there is not yet a clear evidence of the effect that a fully extended $1 \mathrm{~T}^{\prime}$ phase and large single crystalline domains of $1 \mathrm{~T}^{\prime}$ phase can have on the HER performance. For instance, the enhanced catalytic activity in mixed-phase $1 \mathrm{~T}^{\prime} / 2 \mathrm{H}$, as well as in polycrystalline single phase, TMD systems is occasionally attributed to the strained crystalline domain boundaries in the basal plane, providing a higher density of catalytically active sites. ${ }^{227,228}$ Certainly, the bottom-up synthesis approach paves the way to expand the portfolio of $1 \mathrm{~T}^{\prime}$ phases and to control the crystallinity, morphology and crystal phase in multi-layered form (Fig. 8c).

5.2. Increased conductivity for application in energy storage and sensors. Due to the increased electrical conductivity of the metastable $1 \mathrm{~T}$ and $1 \mathrm{~T}^{\prime}$ polymorphs compared to the semiconducting $2 \mathrm{H}$ counterparts, these materials are being extensively explored for energy storage applications. Thus, combined high electrical conductivity and large accessible surface area of metallic TMD nanosheets have been employed in electrochemical double-layer capacitors. ${ }^{101,229} \mathrm{M}$. Acerce et al. have demonstrated that the electrodes formed from restacked chemically exfoliated $1 \mathrm{~T} \mathrm{MoS}_{2}$ nanosheets can intercalate ions, such as $\mathrm{H}^{+}, \mathrm{Li}^{+}, \mathrm{Na}^{+}$, and $\mathrm{K}^{+}$, and can achieve volumetric capacitance of up to $650 \mathrm{~F} \mathrm{~cm}^{-3}$ (at $20 \mathrm{mV} \mathrm{s}^{-1}$ ) in aqueous electrolytes. ${ }^{101}$ Moreover, thus prepared electrodes are suitable for operation in non-aqueous electrolyte (ENIM BF ${ }_{4}$ in $\mathrm{MeCN})$ and demonstrate capacitance as high as $250 \mathrm{~F} \mathrm{~cm}^{-3}$ (at $5 \mathrm{mV} \mathrm{s}^{-1}$ ) and good stability over 5000 cycles $^{101}$ (Fig. 8d).
Grown by solution-phase approach material, exhibiting flowerlike morphology and containing high fraction of metallic $1 \mathrm{~T}$ phase, is gaining interest as a potential electrode material due to high specific surface area accessible to the electrolyte. R. Naz et al. have reported on highly defective $\mathrm{MoS}_{2}$ nanosheets grown on reduced graphene oxide framework achieving high gravimetric capacitance of $442 \mathrm{~F} \mathrm{~g}^{-1}$ (at $1 \mathrm{~A} \mathrm{~g}^{-1}$ ) in aqueous electrolyte $\left(1 \mathrm{M} \mathrm{H}_{2} \mathrm{SO}_{4}\right)$ with capacitance retention of over $84 \%$ during 3000 cycles. $^{230}$ Furthermore, 1T phase $\mathrm{MoS}_{2}$ nanosheets grown through a magneto-hydrothermal reaction deliver a gravimetric capacitance of $379 \mathrm{~F} \mathrm{~g}^{-1}$ (at $1 \mathrm{~A} \mathrm{~g}^{-1}$ ) in $1 \mathrm{M}$ $\mathrm{Li}_{2} \mathrm{SO}_{4}$ and show an exceptionally high stability (nearly 100\%) over 10000 cycles. $^{231}$

Based on first-principle calculations, $1 \mathrm{~T} \mathrm{MoS}_{2}$ monolayers have been identified as a suitable anode material for lithiumion batteries due to their ability to absorb $\mathrm{Li}$ ions at high concentrations without aggregation. ${ }^{232}$ T. Xiang et al. have realised hybrid $1 \mathrm{~T} \mathrm{MoS}_{2}$ /graphene electrodes exhibiting a capacity of $666 \mathrm{~mA} \mathrm{~h} \mathrm{~g}^{-1}$ at high current density $\left(3500 \mathrm{~mA} \mathrm{~g}^{-1}\right){ }^{233}$ It is interesting to note that the arrays of vertically aligned fewlayered $\mathrm{MoS}_{2}$ nanosheets composing the electrodes were grown on graphene via a single-step hydrothermal reaction and the interlayer spacing in thus grown $\mathrm{MoS}_{2}$ reaches $9.8 \AA$ dictated by the graphene lattice; such lattice expansion facilitates a faster Li diffusion essential for a high performance lithium-ion battery. In bulk $\mathrm{MoS}_{2}$ crystals, the repeating lithiation process is reported to form a mosaic-like nanocrystalline domains, which ensure high rate capability and excellent stability in rechargeable lithium-ion batteries. ${ }^{200}$ This process is schematically shown in Fig. 8e. Such phase restructuring in pre-lithiated $\mathrm{MoS}_{2}$ ensures a faster and more stable charge/discharge rate than in either chemically exfoliated or bulk material. ${ }^{200}$ The $1 \mathrm{~T}$ phases have seen recent utilization also in composites for batteries electrodes and we refer the reader to the following reviews for more details on this subject. ${ }^{234-236}$ Covering these works is beyond the scope of the current review as here, we aim at discussing the applications that directly arise from the intrinsic characteristics of the metallic $1 \mathrm{~T}$ phases of group VI TMDs.

The metastable $1 \mathrm{~T}\left(1 \mathrm{~T}^{\prime}\right)$ phases of group VI TMDs have been also proposed to be used in miniaturised biosensors. In particular, chemically exfoliated metallic $1 \mathrm{~T} \mathrm{WS}_{2}$ has been employed as a conductive platform for an indirect enzymatic detection of fenitrothion; the biosensor expressed good linearity in the a broad concentration range $(1-1000 \mathrm{nM})$ with the detection limit as low as $2.86 \mathrm{nM}$ of fenitrothion. ${ }^{201}$ On the other hand, the semimetallic $1 \mathrm{~T}^{\prime} \mathrm{MoTe}_{2}$ and $\mathrm{WTe}_{2}$ polymorphs are reported to be ultrasensitive surface enhanced Raman scattering (SERS) platforms for molecular detection. ${ }^{237}$ In this case, the enhancement of Raman signal is achieved due to the polarisation of analyte molecules (rhodamine 6G) owing to the coupling with the $1 \mathrm{~T}^{\prime}$ phase SERS platform. The reported detection limits are as low as 40 and $400 \mathrm{fM}$ of rhodamine $6 \mathrm{G}$ on pristine few-layered $1 \mathrm{~T}^{\prime} \mathrm{WTe}_{2}$ and $1 \mathrm{~T}^{\prime} \mathrm{MoTe}_{2}$ flakes, respectively; however, these can be further improved when the sensor is integrated on a Bragg reflector.

5.3. Patterned $\mathbf{2 H} / \mathbf{1 T}^{\prime}$ homojunctions for nanoelectronics. Owing to their suitable bandgaps, atomically smooth interfaces, 


\section{Contact resistance engineering for FETs}

a

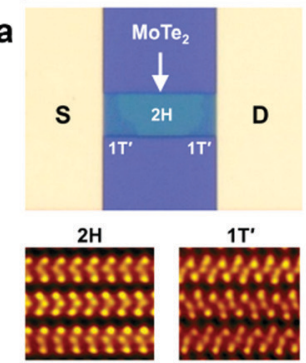

a

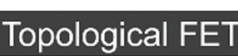

C

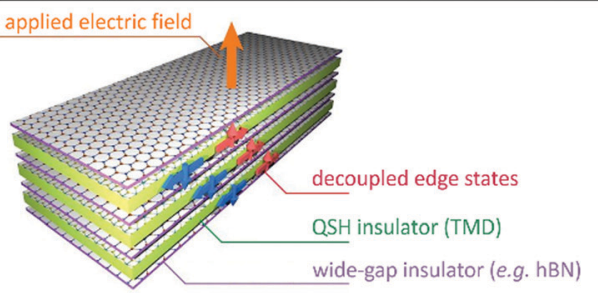

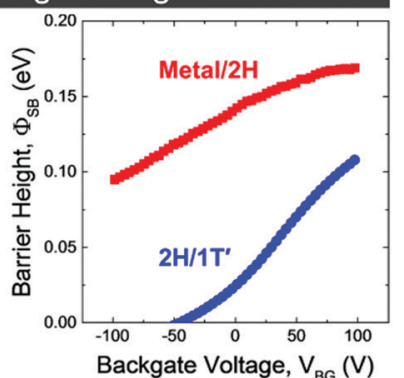

Anisotropic electrical conductivity
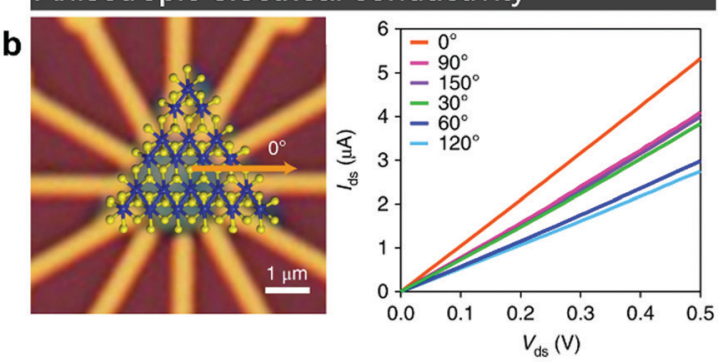

d

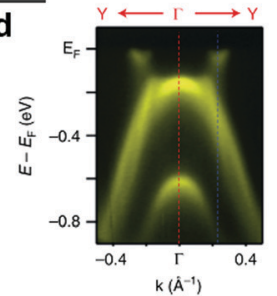

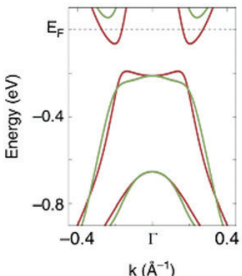

Fig. 9 Applications of the metastable $1 T\left(1 T^{\prime}\right)$ phases of group VI TMDs in novel electronics. (a) Optical image (left) of an FET device with patterned $1 T^{\prime} / 2 \mathrm{H}$ $\mathrm{MoTe}_{2}$ contacts and the Schottky barrier height (right) in the $2 \mathrm{H}$ MoTe $e_{2}$ only device and in the device with patterned $1 \mathrm{~T}^{\prime} / 2 \mathrm{H}$ contacts. (b) Electrical characterisation of a CVD grown $1 \mathrm{~T}^{\prime} \mathrm{MoS}_{2}$ flake (left) demonstrating an anisotropic electrical transport (right). (c) A schematic of a topological FET device based on van der Waals heterostructures of alternating monolayer $1 T^{\prime}$ group VI TMD and single-/few-layer wide-gap insulators. (d) Electronic characterisation of $1 \mathrm{~T}^{\prime} W \mathrm{We}_{2}$ monolayer. High-resolution ARPES band dispersion (left) and the corresponding calculated bands (middle) demonstrate the band inversion in monolayer $1 \mathrm{~T}^{\prime} \mathrm{WS} \mathrm{e}_{2}$; an indirect band gap in $1 \mathrm{~T}^{\prime} \mathrm{WSe} \mathrm{S}_{2}$ calculated from two energy dispersion curves (right) is characteristic to $2 \mathrm{D} \mathrm{TI}$. Separate panels are adapted with permission: (a) from ref. 160, copyright 2019, American Chemical Society; (b) from ref. 147, copyright 2018, Springer Nature; (c) from ref. 46, copyright 2014, American Association for the Advancement of Science; and (d) from ref. 20, copyright 2018, Springer Nature.

and good thermal stability, semiconducting group VI TMDs, and $\mathrm{MoS}_{2}$ single layers in particular, are being investigated for the next generation electronics and optoelectronics. ${ }^{238-241}$ Recently, a field-effect transistor (FET) has been experimentally demonstrated with a single mechanically exfoliated $\mathrm{MoS}_{2}$ flake as a conductive channel. ${ }^{238}$ The device demonstrated an on/off ratio of $10^{8}$ at room temperature and high channel mobilities of at least $200 \mathrm{~cm}^{2} \mathrm{~V}^{-1} \mathrm{~s}^{-1}$ were achieved by dielectric screening with $\mathrm{HfO}_{2}$ as a gate insulator. ${ }^{238}$ The electronics based on atomically thin TMDs is majorly plagued by the large contact resistance between the semiconductor and the source/drain metal electrodes. ${ }^{242}$ This can be solved by locally inducing the $2 \mathrm{H}$-to- $1 \mathrm{~T}^{\prime}$ transformation on TMD nanosheets to significantly lower the contact resistance between the monolayer and bulk metal contacts (Fig. 9a). Local phase patterning to realise ohmic contacts has been demonstrated in $\mathrm{MoS}_{2}$ and $\mathrm{MoTe}_{2}$ transistors. ${ }^{95,155}$ The $1 \mathrm{~T}$ phase electrodes were locally patterned on CVD grown $2 \mathrm{H} \mathrm{MoS}_{2}$ nanosheets by exposing the material to $n$-BuLi, while the semiconducting channel was preserved by protecting this area by a PMMA mask. ${ }^{95}$ In 1-3 layered $\mathrm{MoS}_{2}$ nanosheets, the heterophase $1 \mathrm{~T} / 2 \mathrm{H}$ homojunction is characterised by a contact resistance of $200-300 \Omega \mu \mathrm{m}$ at zero gate bias. Thus fabricated $\mathrm{MoS}_{2}$ FET exhibits the channel mobilities of $\sim 50 \mathrm{~cm}^{2} \mathrm{~V}^{-1} \mathrm{~s}^{-1}$ and on/off ratios exceeding $10^{7} .{ }^{95}$ Similarly, the $1 \mathrm{~T}^{\prime} / 2 \mathrm{H}$ homojunctions induced in $\mathrm{MoTe}_{2}$ by local laser irradiation have increased the channel mobility by a factor of 50 , while preserving the high on/off ratios of $\sim 10^{6}{ }^{6}{ }^{155}$ Interestingly, in $\mathrm{MoTe}_{2}$, lateral $1 \mathrm{~T}^{\prime} / 2 \mathrm{H}$ homojunctions can be obtained via direct CVD synthesis. ${ }^{160,243,244}$ The contact barrier height at the lateral $1 \mathrm{~T}^{\prime} / 2 \mathrm{H}$ interface is reported to be as low as 25-30 meV, ${ }^{160,243}$ and in the coplanar $1 \mathrm{~T}^{\prime} / 2 \mathrm{H}$ contact $\mathrm{MoTe}_{2}$ FET, the channel mobility reached $16.2 \mathrm{~cm}^{2} \mathrm{~V}^{-1} \mathrm{~s}^{-1}$ at room temperature. ${ }^{243}$

5.4. Dynamic phase transitions for non-volatile memory devices. Fast and reversible structural transitions between the crystal phases characterised by a large difference in the electrical resistance between them, lay a foundation for non-volatile memory devices and neuromorphic computing. ${ }^{245}$ F. Zhang et al. have recently demonstrated that the dynamic switching between high resistive $(2 \mathrm{H})$ and low resistive $\left(1 \mathrm{~T}^{\prime}\right)$ states in fewlayered $\mathrm{MoTe}_{2}$ and $\mathrm{W}_{x} \mathrm{Mo}_{1-x} \mathrm{Te}_{2}$ can be controlled by a vertical electric field. ${ }^{43}$ Remarkably, the switching in such resistive random access memory (RRAM) device is achieved within $10 \mathrm{~ns}$ and for the $\mathrm{Al}_{2} \mathrm{O}_{3} / \mathrm{MoTe}_{2}$ stacked configurations, the on/off current ratios are as high as $10^{6}{ }^{43}$ In $\mathrm{MoS}_{2}$ films, a reversible $2 \mathrm{H}$-to- $1 \mathrm{~T}^{\prime}$ phase switching controlled by the in-plane Li ion migration has also been demonstrated. ${ }^{109} \mathrm{~A}$ reversible local phase transformation caused by the difference in $\mathrm{Li}$ ion concentration intercalated between $\mathrm{MoS}_{2}$ layers was effectively modulated by an applied in-plane electric field. Furthermore, it has been shown that the individual devices can be coupled through the local ionic exchange, illustrating their potential for neuromorphic computing. Finally, the material produced in solution phase, such as colloidal $\mathrm{MoS}_{2}$ nanosheets, can be assembled via spray coating, enabling a wafer-scale fabrication of RRAM arrays on flexible substrates for integration in next generation mobile and wearable electronic devices. ${ }^{176}$ 
5.5. Emerging properties and applications. In the semiconducting $2 \mathrm{H}$ polymorph, superconductivity can be induced by intercalation of alkali metals ${ }^{246}$ as was demonstrated in the case of $\mathrm{MoS}_{2}$ with the critical temperatures $T_{\mathrm{c}}$ varying from 1 to $6.5 \mathrm{~K}^{8,247}$ Typically, the fraction $x$ of alkali metal $(\mathrm{A}=\mathrm{Li}, \mathrm{Na}, \mathrm{K}$, $\mathrm{Rb}$ ) in these intercalation compounds $\mathrm{A}_{x} \mathrm{MoS}_{2}$ was as high as $\sim 0.4 .^{247}$ Later, the alkali metal intercalation has been shown to cause the transition into superconducting state in both the $1 \mathrm{~T}$ and $1 \mathrm{~T}^{\prime}$ bulk polymorphs of $\mathrm{MoS}_{2}$ with the $T_{\mathrm{c}}$ of 2.8 and $4.6 \mathrm{~K}$, respectively. ${ }^{248}$ The presence of alkali metal in the intercalated bulk powders was found crucial for the superconducting phase transition. ${ }^{248}$ On the other hand, pristine $1 \mathrm{~T}^{\prime} \mathrm{MoTe}_{2}$ is found to undergo a superconducting phase transition at $0.1 \mathrm{~K}$ and the transition temperature can be dramatically increased to $8.2 \mathrm{~K}$ under applied pressure (11.8 GPa). ${ }^{63}$ Similarly, pressure-driven dome-shaped superconductivity has been reported in bulk WTe $_{2}$ with the highest $T_{\mathrm{c}}(7 \mathrm{~K})$ achieved at $16.8 \mathrm{GPa}^{249}$ Further, dome-shaped superconducting behaviour was observed in pressurised $2 \mathrm{M} \mathrm{WSe}_{2}$ crystals with the resistance drop onset at $4.2 \mathrm{GPa}$ with the $T_{\mathrm{c}}$ of $4.3 \mathrm{~K}$ and the $T_{\mathrm{c}}$ maximum $(7.3 \mathrm{~K})$ at $10.7 \mathrm{GPa}^{72}$ The development of a facile synthesis route to produce bulk $1 \mathrm{~T}\left(1 \mathrm{~T}^{\prime}\right)$ phases of group VI TMDs via gentle deintercalation of $\mathrm{A}_{x} \mathrm{MoS}_{2}$ intercalation compounds has presented an opportunity to study the superconducting phase transition in pure $1 \mathrm{~T}$ TMD polymorph. Thus, $1 \mathrm{~T} \mathrm{MoS}_{2}$ is reported to be an intrinsic superconductor at temperatures below $4 \mathrm{~K}^{143}$

Due to the structural anisotropy in the $a b$ plane, $1 \mathrm{~T}^{\prime}$ single layers have been predicted to exhibit anisotropic transport characteristics. Anisotropic electrical conductivity of the $1 \mathrm{~T}^{\prime}$ phase have been recently demonstrated in chemically exfoliated $^{250}$ and CVD grown $1 \mathrm{~T}^{\prime} \mathrm{MoS}_{2}$ flakes ${ }^{147}$ (Fig. 9b). The conductance reaches its maximum along the $a$ (zigzag) direction and the anisotropic ratio is as high as $2,{ }^{147}$ that exceeds the reported anisotropic conductance for black phosphorus. ${ }^{251}$ This leads to envision application of the $1 \mathrm{~T}^{\prime}$ phases for neuromorphic devices where anisotropic transport is sought in different device components. Black phosphorous, a highly anisotropic 2D material, is being investigated in synaptic devices and higher devices complexities could be enabled by 2D materials with different anisotropic transport.

Bulk nonmagnetic $\mathrm{Td} \mathrm{WTe}_{2}$ demonstrates an extremely large magnetoresistance (XMR); the primary source of which is established to originate from the carrier compensation. ${ }^{252}$ Thus, at $4.5 \mathrm{~K}$, positive magnetoresistance of $452700 \%$ was observed in $\mathrm{Td} \mathrm{WTe}_{2}$ in a magnetic field of $14.7 \mathrm{~T}$; moreover, the magnetoresistance value reaches $13000000 \%$ at $0.53 \mathrm{~K}$ in a magnetic field of $60 \mathrm{~T}$ and no saturation of magnetoresistance is evidenced even in very high magnetic fields. ${ }^{253}$ Later, the XMR behaviour was discovered in the Td phase of $\mathrm{MoTe}_{2} \cdot{ }^{254,255}$ Furthermore, the magnetoresistance observed in $\mathrm{Td} \mathrm{MoTe}_{2}$ in high magnetic fields can be tuned by an applied tensile strain $(0.5 \%) .{ }^{256}$ At low temperatures and in high magnetic fields, the magnetoresistance variation approaches $30 \%$ under uniaxial tensile strain perpendicular to the zigzag chain direction, while the strain along the zigzag chain direction effectively suppresses the magnetoresistance. ${ }^{256}$

\section{Conclusions and future outlook}

In summary, in the past few years there have been exciting fast developments in the elucidation of the potential of the metastable phases of TMDs, ranging from electrocatalysis ${ }^{83,103}$ to condensed matter phenomena such as 2D topological insulators. ${ }^{19}$ Here, we have reviewed the concomitant significant step towards the production of these phases in measurable quantities and the key applications that these phases can uniquely enable. The most widely used method to synthesise metastable phases is based on the intercalation with alkali metals in organic solvent ${ }^{83,114}$ or in a dry form..$^{50,103}$ This is a versatile route which can be applied to bulk powders, with an associated exfoliation of the flakes, or on individual flakes, often CVD grown, on a substrate. Thus, it provides with the ability to produce highly concentrate suspensions of the $1 \mathrm{~T}\left(1 \mathrm{~T}^{\prime}\right)$ phases in water as well as monolayers extended over large areas. The latter is compatible with the integration in planar devices such as field-effect transistors. $^{95,155}$ The first provides stable colloidal suspensions of the $1 \mathrm{~T}$ phases in water which can be processed from solution via vacuum filtration ${ }^{103}$ or dip-coating, ${ }^{257}$ to generate uniform thin films for different applications from electrocatalysis ${ }^{103}$ and photoassisted electrocatalysis, ${ }^{257}$ to energy storage devices. ${ }^{101}$

In the form of thin films, the $1 \mathrm{~T}$ phase can be converted into the $2 \mathrm{H}$ phase upon annealing. ${ }^{27,50}$ Thus, the accessibility of the $2 \mathrm{H}$ phase from the $1 \mathrm{~T}\left(1 \mathrm{~T}^{\prime}\right)$ phase is advantageous as it provides an alternative approach to the commonly used exfoliation of the $2 \mathrm{H}$ phase in organic solvent by applying a shear force, ${ }^{258}$ allowing for the processability of water-based suspensions which are compatible with large-scale production and deposition techniques, such as ink-jet printing, ${ }^{259} 3 \mathrm{D}$ printing, ${ }^{260,261}$ and spray coating, ${ }^{262}$ to name a few.

While chemical treatment has intrinsic advantages and it has been very effective for initial studies of $1 \mathrm{~T}$ phases, as the field progresses, the limitations are now emerging. The first one is represented by the use of Li-chemistry which is air sensitive, involving the use of a controlled atmosphere. Although the intercalation of $\mathrm{MoS}_{2}$ and $\mathrm{WS}_{2}$ bulk powders have demonstrated the $2 \mathrm{H}$-to- $1 \mathrm{~T}\left(1 \mathrm{~T}^{\prime}\right)$ phase transformation, the conversion of the corresponding diselenides $\left(\mathrm{MoSe}_{2}\right.$ and $\mathrm{WSe}_{2}$ ) have not been reported. While the $1 \mathrm{~T}^{\prime}$ phases of $\mathrm{MoSe}_{2}$ and $\mathrm{WSe}_{2}$ have been obtained via direct synthesis, demonstrating that these metastable phases can actually be achieved. ${ }^{68,71}$

Furthermore, the $1 \mathrm{~T}^{\prime}$ phase resulting from the Li intercalation is limited to the $75 \%$ of the material and it has a patchy distribution across the basal plane of individual flakes with a polycrystalline nature, where individual domains can be limited to very few $n m .{ }^{85}$ The impact of the polycrystallinity on the electrical, mechanical and electrocatalytic properties is still not known yet. Thus, the search for bottom-up approaches to the synthesis arises from the need of fully controlling the phase morphology, the materials processability and the possibility to establish a wider spectrum of metastable phases and compounds composition.

Progress has been made in the direct synthesis of metastable phases via both vapour-based deposition techniques and 
in liquid phase from molecular precursors which led to the first demonstrations of the $1 \mathrm{~T}^{\prime}$ phase of $\mathrm{MoS}_{2}{ }^{147}$ and $\mathrm{WSe}_{2} \cdot{ }^{71}$ Most of the reported vapour-phase syntheses are based on chemical vapour transport and have demonstrated the synthesis of bulk crystals of metastable $1 \mathrm{~T}^{\prime} \mathrm{MoSe}_{2}{ }^{68}$ and $1 \mathrm{~T}^{\prime} \mathrm{MoS}_{2}$ phases. ${ }^{68,69}$ The achievement of the $1 \mathrm{~T}^{\prime}$ phase monolayers via CVD approach has been limited to $\mathrm{MoTe}_{2}, \mathrm{WTe}_{2}{ }^{161}$ and $\mathrm{MoS}_{2}{ }^{147}$ These bottom-up approaches lead to nearly continuous $1 \mathrm{~T}^{\prime}$ phase across large areas (over tens of microns) of over $80 \%$ and exhibit a single crystalline nature across hundreds of nanometres. As the atomically thin nature of the flakes is not preserved with CVT synthesis, when bulk crystals are produced, they need to undergo a liquid phase exfoliation step to be used in their 2D form. CVD, which can enable scalability of the synthesis, have the advantage of producing anisotropic monolayers over large areas for application in catalysis, nanoelectronics, neuromorphic systems, quantum technologies and advanced spectroscopies; however, altering the reaction energetics to selectively produce the metastable phase is still challenging.

MBE have been used to demonstrate single crystalline phase of $1 \mathrm{~T}^{\prime} \mathrm{WSe}_{2}{ }^{20,21}$ on bilayer graphene terminated SiC substrates, and of $1 \mathrm{~T}^{\prime} \mathrm{MoSe}_{2}{ }^{70}$ and $1 \mathrm{~T}^{\prime} \mathrm{MoS}_{2}{ }^{79}$ on Au substrates. Although the $1 \mathrm{~T}^{\prime}$ domains are small (less than $100 \mathrm{~nm}$ ), these are sufficiently extended to demonstrate new condensed-matter phenomena so far just predicted, thanks to the single crystalline nature and of the $1 \mathrm{~T}^{\prime}$ phase.

Direct synthesis in solution is the most recent synthesis method demonstrated and it has the advantage of involving low temperatures $\left(<300{ }^{\circ} \mathrm{C}\right)$, high percentage of the $1 \mathrm{~T}^{\prime}$ phase in the final product and single crystalline nature across flakes sizes of $100 \mathrm{~nm} .^{71,80,173}$ Synthesised through a direct colloidal reaction, the nanosheets can form stable dispersions in nonpolar organic solvents. This synthesis route presents features compatible with a large-scale production and the possibility of translating the chemistry of the synthesis into a hydrothermal process.

Branched flower-like nanostructures with atomically thin petals are generally obtained via liquid phase synthesis. ${ }^{71,81,173,199}$ Such morphology is very advantageous for catalysis and supercapacitors as it maximizes the exposure of active sites versus a 2D nanosheets geometry where flakes are planarly stacked, losing some of the accessible surface area. These nanoflowers can also be directly grown on the electrode materials where they can be strongly anchored enabling a good electrical contact. The possibility of obtaining individualized planar 2D nanosheets could, however, expand the applications of the $1 \mathrm{~T}^{\prime}$ phases produced in liquid phase.

The next key challenge lies in understanding the nucleation stage in solution phase to be able to reliably control the flake morphology (between branched sheets and individual sheets) and the crystal phase. As stated by the Ostwald's step rule, which has been based on empirical observations in zeolite materials, the crystallization from a solution occurs in steps in such a way that often thermodynamically unstable phases occur first, followed by a transformation in the thermodynamically stable phase. ${ }^{169}$ Thus, more theoretical understanding and experimental studies of the early stages of nucleation of TMDs are needed to predict the course of the growth and thus to design the ways of controlling morphology and to lock the crystal phase. The ability to produce individualized flakes in solution with micrometric lateral sizes can expand the potential applications competing with CVD methods. Accessing pure $1 \mathrm{~T}$ phases of single crystalline nature across large areas of TMDs is necessary to study the intrinsic physical and chemical properties and so far there is a clear indication that only direct synthesis methods enable to approach $100 \%$ of metastable phase with large single crystals. Also, to fully understand the crystallographic structure of the most exotic metastable $1 \mathrm{~T}^{\prime}$ and $1 \mathrm{~T}^{\prime \prime \prime}$ phases and their related properties is necessary to have highquality single crystals.

For practical applications, the metastable phases should be stabilized in the absence of charged species, i.e. precursor residues or intercalated cations, to produce pristine, kinetically stable materials. To this goal, the Ostwald's step rule could be again useful to predict how to preserve a metastable phase beyond the nucleation point.

The recent evidence that the $1 \mathrm{~T} \mathrm{TMD}_{\mathrm{S}}$ can also be obtained via chemical transformation of non-van der Waals materials, further expands the potential routes of the synthetic approaches possibly making them numerous. ${ }^{152}$ Thus, these new synthesis strategies should pave the way to a new class of atomically thin materials which present new physicochemical features promising in electrocatalytic and energy storage applications as well as new fundamental condensed matter phenomena which can enable new technologies. Recent demonstration of $2 \mathrm{D}$ topological insulating properties in the $1 \mathrm{~T}^{\prime} \mathrm{TMD}$ monolayers could lead to the fabrication of topological field-effect transistors operating at room temperature ${ }^{46}$ (Fig. 9c and d). The anisotropic transport in $1 \mathrm{~T}^{\prime}$ phases dictated by the anisotropic crystal structure could see applications in artificial synaptic devices to emulate biological systems.

The investigation on the exotic physical properties related to the most rarely observed or predicted metastable crystal phases of group VI TMDs $\left(1 \mathrm{~T}^{\prime}\right.$ and $\left.1 \mathrm{~T}^{\prime \prime \prime}\right)$ is progressing very rapidly and there is an incredible potential to exploit these highly anisotropic but stable phases in future nanoelectronics, optoelectronic systems and quantum technology.

\section{Conflicts of interest}

The authors declare no conflict of interest.

\section{Acknowledgements}

The authors would like to acknowledge the award of funding from the European Research Council (ERC) under the European Union's Horizon 2020 research and innovation programme (ERC Consolidator grant agreement no. [819069]) and CM would like to acknowledge the award of a Royal Society University Research Fellowship (UF160539) by the UK Royal Society. 


\section{References}

1 J. A. Wilson and A. D. Yoffe, The transition metal dichalcogenides discussion and interpretation of the observed optical, electrical and structural properties, Adv. Phys., 1969, 18, 193-335.

2 R. B. Murray, R. A. Bromley and A. D. Yoffe, The band structures of some transition metal dichalcogenides. II. Group IVA; octahedral coordination, J. Phys. C: Solid State Phys., 1972, 5, 746-758.

3 R. A. Bromley, R. B. Murray and A. D. Yoffe, The band structures of some transition metal dichalcogenides. III. Group VIA: trigonal prism materials, J. Phys. C: Solid State Phys., 1972, 5, 759-778.

$4 \mathrm{H}$. Tributsch and J. C. Bennett, Electrochemistry and photochemistry of $\mathrm{MoS}_{2}$ layer crystals, J. Electroanal. Chem. Interfacial Electrochem., 1977, 81, 97-111.

$5 \mathrm{H}$. Tributsch, Photoelectrochemical behaviour of layer-type transition metal dichalcogenides, Faraday Discuss. Chem. Soc., 1980, 70, 189.

6 J. A. Baglio, Characterization of n-type semiconducting tungsten disulfide photoanodes in aqueous and nonaqueous electrolyte solutions, J. Electrochem. Soc., 1982, 129, 1461.

7 J. M. Martin, C. Donnet, T. Le Mogne and T. Epicier, Superlubricity of molybdenum disulphide, Phys. Rev. B: Condens. Matter Mater. Phys., 1993, 48, 10583-10586.

8 R. B. Somoano and A. Rembaum, Superconductivity in intercalated molybdenum disulfide, Phys. Rev. Lett., 1971, 27, 402-404.

9 J. A. Woollam and R. B. Somoano, Superconducting critical fields of alkali and alkaline-earth intercalates of $\mathrm{MoS}_{2}$, Phys. Rev. B: Solid State, 1976, 13, 3843-3853.

10 M. Chhowalla, et al., The chemistry of two-dimensional layered transition metal dichalcogenide nanosheets, Nat. Chem., 2013, 5, 263-275.

11 A. Splendiani, et al., Emerging photoluminescence in monolayer $\mathrm{MoS}_{2}$, Nano Lett., 2010, 10, 1271-1275.

12 H. Zeng, J. Dai, W. Yao, D. Xiao and X. Cui, Valley polarization in $\mathrm{MoS}_{2}$ monolayers by optical pumping, Nat. Nanotechnol., 2012, 7, 490-493.

13 W. Zhao, R. M. Ribeiro and G. Eda, Electronic structure and optical signatures of semiconducting transition metal dichalcogenide nanosheets, Acc. Chem. Res., 2015, 48, 91-99.

14 H. Schmidt, F. Giustiniano and G. Eda, Electronic transport properties of transition metal dichalcogenide fieldeffect devices: surface and interface effects, Chem. Soc. Rev., 2015, 44, 7715-7736.

15 D. L. Duong, S. J. Yun and Y. H. Lee, van der Waals layered materials: Opportunities and challenges, ACS Nano, 2017, 11, 11803-11830.

16 S. Manzeli, D. Ovchinnikov, D. Pasquier, O. V. Yazyev and A. Kis, 2D transition metal dichalcogenides, Nat. Rev. Mater., 2017, 2, 17033.

17 T. F. Jaramillo, et al., Identification of active edge sites for electrochemical $\mathrm{H}_{2}$ evolution from $\mathrm{MoS}_{2}$ nanocatalysts, Science, 2007, 317, 100-102.
18 Q. Tang and D. Jiang, Mechanism of hydrogen evolution reaction on $1 \mathrm{~T}-\mathrm{MoS}_{2}$ from first principles, ACS Catal., 2016, 6, 4953-4961.

19 S. Tang, et al., Quantum spin Hall state in monolayer 1T'-WTe ${ }_{2}$, Nat. Phys., 2017, 13, 683-687.

20 M. M. Ugeda, et al., Observation of topologically protected states at crystalline phase boundaries in single-layer $\mathrm{WSe}_{2}$, Nat. Commun., 2018, 9, 3401.

$21 \mathrm{P}$. Chen, et al., Large quantum-spin-Hall gap in single-layer $1 \mathrm{~T}^{\prime} \mathrm{WSe}_{2}$, Nat. Commun., 2018, 9, 2003.

22 Y. Xu, et al., Large-gap quantum spin Hall insulators in tin films, Phys. Rev. Lett., 2013, 111, 136804.

23 D. V. Gruznev, et al., Two-dimensional In-Sb compound on silicon as a quantum spin Hall insulator, Nano Lett., 2018, 18, 4338-4345.

24 S. N. Shirodkar and U. V. Waghmare, Emergence of ferroelectricity at a metal-semiconductor transition in a $1 \mathrm{~T}$ monolayer of $\mathrm{MoS}_{2}$, Phys. Rev. Lett., 2014, 112, 157601.

25 K.-A. N. Duerloo, Y. Li and E. J. Reed, Structural phase transitions in two-dimensional Mo- and W-dichalcogenide monolayers, Nat. Commun., 2014, 5, 1-9.

26 P. Joensen, R. F. Frindt and S. R. Morrison, Single-layer $\mathrm{MoS}_{2}$, Mater. Res. Bull., 1986, 21, 457-461.

27 F. Wypych and R. Schöllhorn, 1T- $\mathrm{MoS}_{2}$, a new metallic modification of molybdenum disulfide, J. Chem. Soc., Chem. Commun., 1992, 1386-1388.

28 X. Zhao, S. Ning, W. Fu, S. J. Pennycook and K. P. Loh, Differentiating polymorphs in molybdenum disulfide via electron microscopy, Adv. Mater., 2018, 30, 1802397.

29 D. Puotinen and R. E. Newnham, The crystal structure of $\mathrm{MoTe}_{2}$, Acta Crystallogr., 1961, 14, 691-692.

30 P. B. James and M. T. Lavik, The crystal structure of $\mathrm{MoSe}_{2}$, Acta Crystallogr., 1963, 16, 1183.

31 R. G. Dickinson and L. Pauling, The crystal structure of molybdenite, J. Am. Chem. Soc., 1923, 45, 1466-1471.

32 W. G. Fisher and M. J. Sienko, Stoichiometry, structure, and physical properties of niobium disulfide, Inorg. Chem., 1980, 19, 39-43.

33 X. Fan, D. J. Singh, Q. Jiang and W. T. Zheng, Pressure evolution of the potential barriers of phase transition of $\mathrm{MoS}_{2}, \mathrm{MoSe}_{2}$ and $\mathrm{MoTe}_{2}$, Phys. Chem. Chem. Phys., 2016, 18, 12080-12085.

34 A. P. Nayak, et al., Pressure-induced semiconducting to metallic transition in multilayered molybdenum disulphide, Nat. Commun., 2014, 5, 3731.

35 Z.-H. Chi, et al., Pressure-induced metallization of molybdenum disulfide, Phys. Rev. Lett., 2014, 113, 036802.

36 D. Xiao, G.-B. Liu, W. Feng, X. Xu and W. Yao, Coupled spin and valley physics in monolayers of $\mathrm{MoS}_{2}$ and other group-VI dichalcogenides, Phys. Rev. Lett., 2012, 108, 196802.

37 K. F. Mak, K. He, J. Shan and T. F. Heinz, Control of valley polarization in monolayer $\mathrm{MoS}_{2}$ by optical helicity, Nat. Nanotechnol., 2012, 7, 494-498.

38 A. M. Jones, et al., Optical generation of excitonic valley coherence in monolayer $\mathrm{WSe}_{2}$, Nat. Nanotechnol., 2013, 8, 634-638. 
39 R. Suzuki, et al., Valley-dependent spin polarization in bulk $\mathrm{MoS}_{2}$ with broken inversion symmetry, Nat. Nanotechnol., 2014, 9, 611-617.

$40 \mathrm{~J}$. Shi, et al., 3R $\mathrm{MoS}_{2}$ with broken inversion symmetry: A promising ultrathin nonlinear optical device, Adv. Mater., 2017, 29, 1701486.

41 Z. Zeng, et al., Controlled vapor growth and nonlinear optical applications of large-area $3 \mathrm{R}$ phase $\mathrm{WS}_{2}$ and $\mathrm{WSe}_{2}$ atomic layers, Adv. Funct. Mater., 2019, 29, 1806874.

42 Y.-C. Lin, D. O. Dumcenco, Y.-S. Huang and K. Suenaga, Atomic mechanism of the semiconducting-to-metallic phase transition in single-layered $\mathrm{MoS}_{2}$, Nat. Nanotechnol., 2014, 9, 391-396.

43 F. Zhang, et al., Electric-field induced structural transition in vertical $\mathrm{MoTe}_{2}$ - and $\mathrm{Mo}_{1-x} \mathrm{~W}_{x} \mathrm{Te}_{2}$-based resistive memories, Nat. Mater., 2019, 18, 55-61.

44 R. B. Murray and A. D. Yoffe, The band structures of some transition metal dichalcogenides: band structures of the titanium dichalcogenides, J. Phys. C: Solid State Phys., 1972, 5, 3038-3046.

45 A. Singh, S. N. Shirodkar and U. V. Waghmare, $1 \mathrm{H}$ and $1 \mathrm{~T}$ polymorphs, structural transitions and anomalous properties of $(\mathrm{Mo}, \mathrm{W})(\mathrm{S}, \mathrm{Se})_{2}$ monolayers: first-principles analysis, 2D Mater., 2015, 2, 035013.

46 X. Qian, J. Liu, L. Fu and J. Li, Quantum spin Hall effect in two-dimensional transition metal dichalcogenides, Science, 2014, 346, 1344-1347.

47 J. Heising and M. G. Kanatzidis, Structure of restacked $\mathrm{MoS}_{2}$ and $\mathrm{WS}_{2}$ elucidated by electron crystallography, J. Am. Chem. Soc., 1999, 121, 638-643.

48 G. Gao, et al., Charge mediated semiconducting-tometallic phase transition in molybdenum disulfide monolayer and hydrogen evolution reaction in new $1 \mathrm{~T}^{\prime}$ phase, J. Phys. Chem. C, 2015, 119, 13124-13128.

49 X. R. Qin, D. Yang, R. F. Frindt and J. C. Irwin, Real-space imaging of single-layer $\mathrm{MoS}_{2}$ by scanning tunneling microscopy, Phys. Rev. B: Condens. Matter Mater. Phys., 1991, 44, 3490-3493.

50 H. L. Tsai, J. Heising, J. L. Schindler, C. R. Kannewurf and M. G. Kanatzidis, Exfoliated-restacked phase of $\mathrm{WS}_{2}$, Chem. Mater., 1997, 9, 879-882.

51 K. K. Amara, et al., Dynamic structural evolution of metalmetal bonding network in monolayer $\mathrm{WS}_{2}$, Chem. Mater., 2016, 28, 2308-2314.

52 W. Zhao, et al., Metastable $\mathrm{MoS}_{2}$ : Crystal structure, electronic band structure, synthetic approach and intriguing physical properties, Chem. - Eur. J., 2018, 24, 15942-15954.

53 H. L. Zhuang, M. D. Johannes, A. K. Singh and R. G. Hennig, Doping-controlled phase transitions in singlelayer $\mathrm{MoS}_{2}$, Phys. Rev. B, 2017, 96, 165305.

54 E. Bruyer, et al., Possibility of combining ferroelectricity and Rashba-like spin splitting in monolayers of the 1T-type transition-metal dichalcogenides $\mathrm{MX}_{2}(\mathrm{M}=\mathrm{Mo}, \mathrm{W} ; \mathrm{X}=\mathrm{S}$, Se, Te), Phys. Rev. B, 2016, 94, 195402.

55 C. Rovira and M. H. Whangbo, Factors governing the charge density wave patterns of layered transition-metal compounds of octahedral coordination with $\mathrm{d} 2$ and $\mathrm{d} 3$ electron counts, Inorg. Chem., 1993, 32, 4094-4097.

56 Y. Fang, et al., Structural determination and nonlinear optical properties of new $1 \mathrm{~T}^{\prime \prime \prime}$-type $\mathrm{MoS}_{2}$ compound, J. Am. Chem. Soc., 2019, 141, 790-793.

57 B. E. Brown, The crystal structures of $\mathrm{WTe}_{2}$ and hightemperature $\mathrm{MoTe}_{2}$, Acta Crystallogr., 1966, 20, 268-274.

58 L. Zhou, et al., Sensitive phonon-based probe for structure identification of $1 \mathrm{~T}^{\prime} \mathrm{MoTe}_{2}, \mathrm{~J}$. Am. Chem. Soc., 2017, 139, 8396-8399.

59 J. C. Park, et al., Phase-engineered synthesis of centimeterscale $1 \mathrm{~T}^{\prime}$ - and $2 \mathrm{H}$-molybdenum ditelluride thin films, ACS Nano, 2015, 9, 6548-6554.

60 Y. Yoo, Z. P. DeGregorio, Y. Su, S. J. Koester and J. E. Johns, In-plane $2 \mathrm{H}-1 \mathrm{~T}^{\prime} \quad \mathrm{MoTe}_{2}$ homojunctions synthesized by flux-controlled phase engineering, Adv. Mater., 2017, 29, 1605461.

61 W. G. Dawson and D. W. Bullett, Electronic structure and crystallography of $\mathrm{MoTe}_{2}$ and $\mathrm{WTe}_{2}, J$. Phys. C: Solid State Phys., 1987, 20, 6159-6174.

62 S. Chen, T. Goldstein, D. Venkataraman, A. Ramasubramaniam and J. Yan, Activation of new Raman modes by inversion symmetry breaking in type II Weyl semimetal candidate $\mathrm{T}^{\prime}$-MoTe ${ }_{2}$, Nano Lett., 2016, 16, 5852-5860.

63 Y. Qi, et al., Superconductivity in Weyl semimetal candidate $\mathrm{MoTe}_{2}$, Nat. Commun., 2016, 7, 11038.

$64 \mathrm{~J}$. Jiang, et al., Signature of type-II Weyl semimetal phase in $\mathrm{MoTe}_{2}$, Nat. Commun., 2017, 8, 13973.

65 D. Rhodes, et al., Engineering the structural and electronic phases of $\mathrm{MoTe}_{2}$ through W substitution, Nano Lett., 2017, 17, 1616-1622.

66 Y. Zhou, et al., Pressure-induced Td to $1 \mathrm{~T}^{\prime}$ structural phase transition in $\mathrm{WTe}_{2}$, AIP Adv., 2016, 6, 075008.

67 J. Xia, et al., Pressure-induced phase transition in Weyl semimetallic $\mathrm{WTe}_{2}$, Small, 2017, 13, 1701887.

$68 \mathrm{Y}$. Yu, et al., High phase-purity $1 \mathrm{~T}^{\prime}-\mathrm{MoS}_{2^{-}}$and $1 \mathrm{~T}^{\prime}-\mathrm{MoSe}_{2^{-}}$ layered crystals, Nat. Chem., 2018, 10, 638-643.

$69 \mathrm{~J}$. Peng, et al., High phase purity of large-sized $1 \mathrm{~T}^{\prime}-\mathrm{MoS}_{2}$ monolayers with 2D superconductivity, Adv. Mater., 2019, 31, 1900568.

70 F. Cheng, et al., Interface Engineering of $\mathrm{Au}(111)$ for the Growth of $1 \mathrm{~T}^{\prime}-\mathrm{MoSe}_{2}$, ACS Nano, 2019, 13, 2316-2323.

71 M. S. Sokolikova, P. C. Sherrell, P. Palczynski, V. L. Bemmer and C. Mattevi, Direct solution-phase synthesis of $1 \mathrm{~T}^{\prime} \mathrm{WSe}_{2}$ nanosheets, Nat. Commun., 2019, 10, 712.

72 Y. Fang, et al., Observation of superconductivity in pressurized $2 \mathrm{M} \mathrm{WSe}_{2}$ crystals, J. Mater. Chem. C, 2019, 7, 8551-8555.

73 Y. Sun, S.-C. Wu, M. N. Ali, C. Felser and B. Yan, Prediction of Weyl semimetal in orthorhombic $\mathrm{MoTe}_{2}$, Phys. Rev. B: Condens. Matter Mater. Phys., 2015, 92, 161107.

74 Z. Wang, et al., $\mathrm{MoTe}_{2}$ : A type-II Weyl topological metal, Phys. Rev. Lett., 2016, 117, 056805.

$75 \mathrm{P} . \mathrm{Li}$, et al., Evidence for topological type-II Weyl semimetal $\mathrm{WTe}_{2}$, Nat. Commun., 2017, 8, 2150.

$76 \mathrm{Y} . \mathrm{Wu}$, et al., Observation of Fermi arcs in the type-II Weyl semimetal candidate $\mathrm{WTe}_{2}$, Phys. Rev. B, 2016, 94, 121113. 
77 K. Deng, et al., Experimental observation of topological Fermi arcs in type-II Weyl semimetal $\mathrm{MoTe}_{2}$, Nat. Phys., 2016, 12, 1105-1110.

78 A. Pulkin and O. V. Yazyev, Robustness of the quantum spin Hall insulator phase in monolayer $1 \mathrm{~T}^{\prime}$ transition metal dichalcogenides, J. Electron Spectrosc. Relat. Phenom., 2017, 219, 72-76.

$79 \mathrm{H}$. Xu, et al., Observation of gap opening in $1 \mathrm{~T}^{\prime}$ phase $\mathrm{MoS}_{2}$ nanocrystals, Nano Lett., 2018, 18, 5085-5090.

80 B. Mahler, V. Hoepfner, K. Liao and G. A. Ozin, Colloidal synthesis of $1 \mathrm{~T}-\mathrm{WS}_{2}$ and $2 \mathrm{H}-\mathrm{WS}_{2}$ nanosheets: Applications for photocatalytic hydrogen evolution, J. Am. Chem. Soc., 2014, 136, 14121-14127.

81 Q. Liu, et al., Gram-scale aqueous synthesis of stable fewlayered $1 \mathrm{~T}-\mathrm{MoS}_{2}$ : Applications for visible-light-driven photocatalytic hydrogen evolution, Small, 2015, 11, 5556-5564.

82 Q. Liu, et al., Stable metallic $1 \mathrm{~T}-\mathrm{WS}_{2}$ nanoribbons intercalated with ammonia ions: The correlation between structure and electrical/optical properties, Adv. Mater., 2015, 27, 4837-4844.

83 D. Voiry, et al., Enhanced catalytic activity in strained chemically exfoliated $\mathrm{WS}_{2}$ nanosheets for hydrogen evolution, Nat. Mater., 2013, 12, 850-855.

84 Y. Guo, et al., Probing the dynamics of the metallic-tosemiconducting structural phase transformation in $\mathrm{MoS}_{2}$ crystals, Nano Lett., 2015, 15, 5081-5088.

85 G. Eda, et al., Coherent atomic and electronic heterostructures of single-layer $\mathrm{MoS}_{2}$, ACS Nano, 2012, 6, 7311-7317.

86 S. J. R. Tan, et al., Chemical stabilization of $1 \mathrm{~T}^{\prime}$ phase transition metal dichalcogenides with giant optical Kerr nonlinearity, J. Am. Chem. Soc., 2017, 139, 2504-2511.

87 S. Jiménez Sandoval, D. Yang, R. F. Frindt and J. C. Irwin, Raman study and lattice dynamics of single molecular layers of $\mathrm{MoS}_{2}$, Phys. Rev. B: Condens. Matter Mater. Phys., 1991, 44, 3955-3962.

$88 \mathrm{X} . \mathrm{Ma}$, et al., Raman scattering in the transition-metal dichalcogenides of $1 \mathrm{~T}^{\prime}-\mathrm{MoTe}_{2}, \mathrm{Td}-\mathrm{MoTe}_{2}$, and $\mathrm{Td}-\mathrm{WTe} \mathrm{T}_{2}$, Phys. Rev. B, 2016, 94, 214105.

89 S.-Y. Chen, C. H. Naylor, T. Goldstein, A. T. C. Johnson and J. Yan, Intrinsic Phonon Bands in High-Quality Monolayer $\mathrm{T}^{\prime}$ Molybdenum Ditelluride, ACS Nano, 2017, 11, 814-820.

90 C.-H. Lee, et al., Tungsten ditelluride: a layered semimetal, Sci. Rep., 2015, 5, 10013.

91 S. J. R. Tan, et al., Temperature- and Phase-Dependent Phonon Renormalization in $1 \mathrm{~T}^{\prime}-\mathrm{MoS}_{2}$, ACS Nano, 2018, 12, 5051-5058.

92 D. Pierucci, et al., Evidence for a narrow band gap phase in $1 \mathrm{~T}^{\prime} \mathrm{WS}_{2}$ nanosheet, Appl. Phys. Lett., 2019, 115, 032102.

93 C. Guo, et al., Observation of superconductivity in $1 \mathrm{~T}^{\prime}$ $\mathrm{MoS}_{2}$ nanosheets, J. Mater. Chem. C, 2017, 5, 10855-10860.

$94 \mathrm{X}$. Fan, et al., Fast and efficient preparation of exfoliated $2 \mathrm{H} \mathrm{MoS}$ nanosheets by sonication-assisted lithium intercalation and infrared laser-induced $1 \mathrm{~T}$ to $2 \mathrm{H}$ phase reversion, Nano Lett., 2015, 15, 5956-5960.

95 A. D. Mohite and M. Chhowalla, Phase-engineered lowresistance contacts for ultrathin $\mathrm{MoS}_{2}$ transistors, Nat. Mater., 2014, 13, 1128-1134.
96 R. Kappera, et al., Metallic 1T phase source/drain electrodes for field effect transistors from chemical vapor deposited $\mathrm{MoS}_{2}$ transistors from chemical vapor deposited $\mathrm{MoS}_{2}, A P L$ Mater., 2014, 2, 092516.

97 Y. Ma, et al., Reversible semiconducting-to-metallic phase transition in chemical vapor deposition grown monolayer $\mathrm{WSe}_{2}$ and applications for devices, ACS Nano, 2015, 9, 7383-7391.

98 D. Voiry, A. Mohite and M. Chhowalla, Phase engineering of transition metal dichalcogenides, Chem. Soc. Rev., 2015, 44, 2702-2712.

99 L. Niu, et al., Production of Two-Dimensional Nanomaterials via Liquid-Based Direct Exfoliation, Small, 2016, 12, 272-293.

100 D. M. Sim, et al., Long-term stable $2 \mathrm{H}-\mathrm{MoS}_{2}$ dispersion: Critical role of solvent for simultaneous phase restoration and surface functionalization of liquid-exfoliated $\mathrm{MoS}_{2}$, ACS Omega, 2017, 2, 4678-4687.

101 M. Acerce, D. Voiry and M. Chhowalla, Metallic 1T phase $\mathrm{MoS}_{2}$ nanosheets as supercapacitor electrode materials, Nat. Nanotechnol., 2015, 10, 313-318.

102 A. Ambrosi, Z. Sofer and M. Pumera, 2H $\rightarrow$ 1T phase transition and hydrogen evolution activity of $\mathrm{MoS}_{2}, \mathrm{MoSe}_{2}$, $\mathrm{WS}_{2}$ and $\mathrm{WSe}_{2}$ strongly depends on the MX2 composition, Chem. Commun., 2015, 51, 8450-8453.

103 D. Voiry, et al., Conducting $\mathrm{MoS}_{2}$ nanosheets as catalysts for hydrogen evolution reaction, Nano Lett., 2013, 13, 6222-6227.

104 L. Wang, Z. Xu, W. Wang and X. Bai, Atomic mechanism of dynamic electrochemical lithiation processes of MoS2 nanosheets, J. Am. Chem. Soc., 2014, 136, 6693-6697.

105 D. Yang, S. J. Sandoval, W. M. R. Divigalpitiya, J. C. Irwin and R. F. Frindt, Structure of single-molecular-layer MoS2, Phys. Rev. B: Condens. Matter Mater. Phys., 1991, 43, 12053-12056.

106 K. K. Bardhan, J. C. Wu, J. S. Culik, S. H. Anderson and D. D. L. Chung, Kinetics of intercalation and desorption in graphite, Synth. Met., 1980, 2, 57-84.

107 M. Azhagurajan, T. Kajita, T. Itoh, Y.-G. Kim and K. Itaya, In situ visualization of lithium ion intercalation into MoS2 single crystals using differential optical microscopy with atomic layer resolution, J. Am. Chem. Soc., 2016, 138, 3355-3361.

108 Z. Zeng, et al., Single-layer semiconducting nanosheets: High-yield preparation and device fabrication, Angew. Chem., Int. Ed., 2011, 50, 11093-11097.

109 X. Zhu, D. Li, X. Liang and W. D. Lu, Ionic modulation and ionic coupling effects in MoS2 devices for neuromorphic computing, Nat. Mater., 2019, 18, 141-148.

110 F. Xiong, et al., Li intercalation in MoS2: In situ observation of its dynamics and tuning optical and electrical properties, Nano Lett., 2015, 15, 6777-6784.

$111 \mathrm{~J}$. Zheng, et al., High yield exfoliation of two-dimensional chalcogenides using sodium naphthalenide, Nat. Commun., 2014, 5, 2995.

112 C. Tan, et al., Preparation of high-percentage 1T-phase transition metal dichalcogenide nanodots for electrochemical hydrogen evolution, Adv. Mater., 2018, 30, 1705509. 
113 M. Naz, et al., A new $2 \mathrm{H}-2 \mathrm{H}^{\prime} / 1 \mathrm{~T}$ cophase in polycrystalline MoS2 and MoSe2 thin films, ACS Appl. Mater. Interfaces, 2016, 8, 31442-31448.

114 L. Sun, et al., Layer-dependent chemically induced phase transition of two-dimensional MoS2, Nano Lett., 2018, 18, 3435-3440.

115 A. Y. S. Eng, A. Ambrosi, Z. Sofer, P. Šimek and M. Pumera, Electrochemistry of transition metal dichalcogenides: Strong dependence on the metal-to-chalcogen composition and exfoliation method, ACS Nano, 2014, 8, 12185-12198.

116 A. L. Friedman, et al., Evidence for chemical vapor induced $2 \mathrm{H}$ to $1 \mathrm{~T}$ phase transition in $\mathrm{MoX} 2(\mathrm{X}=\mathrm{Se}, \mathrm{S})$ transition metal dichalcogenide films, Sci. Rep., 2017, 7, 3836.

117 M. Wuttig and N. Yamada, Phase-change materials for rewriteable data storage, Nat. Mater., 2007, 6, 824-832.

118 J.-J. Kim, et al., Observation of a phase transition from the $\mathrm{T}$ phase to the $\mathrm{H}$ phase induced by a STM tip in 1T-TaS2, Phys. Rev. B: Condens. Matter Mater. Phys., 1997, 56, R15573-R15576.

119 Y. Li, K.-A. N. Duerloo, K. Wauson and E. J. Reed, Structural semiconductor-to-semimetal phase transition in twodimensional materials induced by electrostatic gating, Nat. Commun., 2016, 7, 10671.

120 Y. Wang, et al., Structural phase transition in monolayer MoTe2 driven by electrostatic doping, Nature, 2017, 550, 487-491.

121 C. Zhang, et al., Charge mediated reversible metalinsulator transition in monolayer $\mathrm{MoTe}_{2}$ and $\mathrm{W}_{x} \mathrm{Mo}_{1-x} \mathrm{Te}_{2}$ alloy, ACS Nano, 2016, 10, 7370-7375.

122 Y. Sun, Z. Shuai and D. Wang, Janus monolayer of WSeTe, a new structural phase transition material driven by electrostatic gating, Nanoscale, 2018, 10, 21629-21633.

123 S. Kim, et al., Long-range lattice engineering of MoTe2 by a 2D electride, Nano Lett., 2017, 17, 3363-3368.

124 J. L. Dye, Electrons as Anions, Nature, 2003, 301, 607-609.

125 K. Lee, S. W. Kim, Y. Toda, S. Matsuishi and H. Hosono, Dicalcium nitride as a two-dimensional electride with an anionic electron layer, Nature, 2013, 494, 336-340.

126 D. L. Dru, et al., Experimental demonstration of an electride as a 2D material, J. Am. Chem. Soc., 2016, 138, 16089-16094.

127 Y. Kang, et al., Plasmonic hot electron induced structural phase transition in a MoS2 monolayer, Adv. Mater., 2014, 26, 6467-6471.

$128 \mathrm{X}$. Yin, et al., Tunable inverted gap in monolayer quasimetallic MoS2 induced by strong charge-lattice coupling, Nat. Commun., 2017, 8, 486.

129 H. H. Huang, et al., Controlling phase transition for singlelayer MTe2 (M = Mo and $\mathrm{W})$ : modulation of the potential barrier under strain, Phys. Chem. Chem. Phys., 2016, 18, 4086-4094.

130 S. Song, et al., Room temperature semiconductor-metal transition of MoTe2 thin films engineered by strain, Nano Lett., 2015, 16, 188-193.

131 C. H. Sharma, A. P. Surendran, A. Varghese and M. Thalakulam, Stable and scalable 1T MoS2 with low temperature-coefficient of resistance, Sci. Rep., 2018, 8, 12463.

132 S. Reshmi, M. V. Akshaya, B. Satpati, P. K. Basu and K. Bhattacharjee, Structural stability of coplanar 1T-2H superlattice MoS2 under high energy electron beam, Nanotechnology, 2018, 29, 205604.

133 Y. Qi, et al., CO2-Induced Phase Engineering: Protocol for Enhanced Photoelectrocatalytic Performance of 2D MoS2 Nanosheets, ACS Nano, 2016, 10, 2903-2909.

134 X. Tong, Y. Qi, J. Chen, N. Wang and Q. Xu, Supercritical CO2-Assisted Reverse-Micelle-Induced Solution-Phase Fabrication of Two-Dimensional Metallic 1T-MoS2 and 1T-WS2, ChemNanoMat, 2017, 3, 466-471.

$135 \mathrm{X}$. Gan, et al., 2H/1T phase transition of multilayer MoS2 by electrochemical incorporation of S vacancies, ACS Appl. Energy Mater., 2018, 1, 4754-4765.

136 W. Ding, et al., Highly Ambient-Stable $1 \mathrm{~T}-\mathrm{MoS}_{2}$ and 1T-WS ${ }_{2}$ by Hydrothermal Synthesis under High Magnetic Fields, ACS Nano, 2019, 13, 1694-1702.

137 Y. Tan, et al., Controllable $2 \mathrm{H}$-to- $1 \mathrm{~T}^{\prime}$ phase transition in few-layer MoTe2, Nanoscale, 2018, 10, 19964-19971.

$138 \mathrm{~J}$. Zhu, et al., Argon plasma induced phase transition in monolayer MoS2, J. Am. Chem. Soc., 2017, 139, 10216-10219.

139 Z. Wang, et al., Local engineering of topological phase in monolayer MoS2, Sci. Bull., 2019, 64, 1750-1756.

$140 \mathrm{H}$. Mine, et al., Laser-beam-patterned topological insulating states on thin semiconducting MoS2, Phys. Rev. Lett., 2019, 123, 146803.

141 Z. Wang, et al., Synthesizing $1 \mathrm{~T}-1 \mathrm{H}$ two-phase $\mathrm{Mo}_{1-x} \mathrm{~W}_{x} \mathrm{~S}_{2}$ monolayers by chemical vapor deposition, ACS Nano, 2018, 12, 1571-1579.

142 F. Wypych, T. Weber and R. Prins, Scanning tunneling microscopic investigation of $\mathrm{K}_{x}\left(\mathrm{H}_{2} \mathrm{O}\right)_{y} \mathrm{MoS}_{2}$, Surf. Sci., 1997, 380, L474-L478.

143 Y. Fang, et al., Structure re-determination and superconductivity observation of bulk $1 \mathrm{~T}$ MoS2, Angew. Chem., 2018, 130, 1246-1249.

144 C. Guo, et al., High-quality single-layer nanosheets of MS2 $(\mathrm{M}=\mathrm{Mo}, \mathrm{Nb}, \mathrm{Ta}, \mathrm{Ti})$ directly exfoliated from AMS2 $(\mathrm{A}=\mathrm{Li}$, Na, K) Crystals, J. Mater. Chem. C, 2017, 5, 5977-5983.

145 F. Wypych, C. Solenthaler, R. Prins and T. Weber, Electron diffraction study of intercalation compounds derived from 1T-MoS2, J. Solid State Chem., 1999, 436, 430-436.

146 F. Wypych, T. Weber and R. Prins, Scanning tunneling microscopic investigation of 1T-MoS2, Surf. Sci., 1998, 380, L474-L478.

147 L. Liu, et al., Phase-selective synthesis of $1 \mathrm{~T}^{\prime}$ MoS2 monolayers and heterophase bilayers, Nat. Mater., 2018, 17, 1108-1114.

148 Z. Liu, et al., Colloidal synthesis of $1 \mathrm{~T}^{\prime}$ phase dominated WS2 towards endurable electrocatalysis, Nano Energy, 2018, 50, 176-181.

149 J. Ekspong, et al., Stable sulfur-intercalated $1 \mathrm{~T}^{\prime}$ MoS2 on graphitic nanoribbons as hydrogen evolution electrocatalyst, Adv. Funct. Mater., 2018, 28, 1802744. 
150 Q. Liu, et al., Electron-doped 1T-MoS2 via interface engineering for enhanced electrocatalytic hydrogen evolution, Chem. Mater., 2017, 29, 4738-4744.

$151 \mathrm{~J}$. Tang, et al., MXene derived TiS2 nanosheets for high-rate and long-life sodium-ion capacitors, Energy Storage Mater., 2020, 26, 550-559.

152 Z. Du, et al., Conversion of non-van der Waals solids to 2D transition-metal chalcogenides, Nature, 2020, 577, 492-496.

153 D. H. Keum, et al., Bandgap opening in few-layered monoclinic MoTe2, Nat. Phys., 2015, 11, 482-486.

$154 \mathrm{X} . \mathrm{Xu}$, et al., Millimeter-Scale Single-Crystalline Semiconducting MoTe2 via Solid-to-Solid Phase Transformation, J. Am. Chem. Soc., 2019, 141, 2128-2134.

155 S. Cho, et al., Phase patterning for ohmic homojunction contact in MoTe2, Science, 2015, 349, 625-628.

156 L. Zhou, et al., Synthesis of high-quality large-area homogenous $1 \mathrm{~T}^{\prime}$ MoTe2 from chemical vapor deposition, $A d v$. Mater., 2016, 28, 9526-9531.

157 L. Zhou, et al., Large-area synthesis of high-quality uniform few-layer MoTe2, J. Am. Chem. Soc., 2015, 137, 11892-11895.

158 L. Zhou, et al., Role of molecular sieves in the CVD synthesis of large-area 2D MoTe2, Adv. Funct. Mater., 2017, 27, 1603491.

159 L. Yang, et al., Tellurization velocity-dependent metallicsemiconducting-metallic phase evolution in chemical vapor deposition growth of large-area, few-layer MoTe2, ACS Nano, 2017, 11, 1964-1972.

160 R. Ma, et al., MoTe2 lateral homojunction field-effect transistors fabricated using flux-controlled phase engineering, ACS Nano, 2019, 13, 8035-8046.

$161 \mathrm{~J}$. Zhou, et al., Large-Area and High-Quality 2D Transition Metal Telluride, Adv. Mater., 2017, 29, 1603471.

162 R. Sankar, et al., Polymorphic layered MoTe2 from semiconductor, topological insulator, to Weyl semimetal, Chem. Mater., 2017, 29, 699-707.

163 T. A. Empante, et al., Chemical vapor deposition growth of few-layer MoTe2 in the $2 \mathrm{H}, 1 \mathrm{~T}^{\prime}$, and $1 \mathrm{~T}$ phases: Tunable properties of MoTe2 films, ACS Nano, 2017, 11, 900-905.

164 Y.-C. Lin, et al., Stable 1T tungsten disulfide monolayer and its junctions: Growth and atomic structures, ACS Nano, 2018, 12, 12080-12088.

165 B.-R. Chen, et al., Understanding crystallization pathways leading to manganese oxide polymorph formation, Nat. Commun., 2018, 9, 2553.

166 S. Lee, et al., Multiple pathways of crystal nucleation in an extremely supersaturated aqueous potassium dihydrogen phosphate (KDP) solution droplet, Proc. Natl. Acad. Sci. U. S. A., 2016, 113, 13618-13623.

$167 \mathrm{H}$. Zhang and J. F. Banfield, Understanding polymorphic phase transformation behavior during growth of nanocrystalline aggregates: Insights from TiO2, J. Phys. Chem. B, 2000, 104, 3481-3487.

168 J. D. Dunitz and J. Bernstein, Disappearing polymorphs, Acc. Chem. Res., 1995, 28, 193-200.

169 R. A. Van Santen, The Ostwald step rule, J. Phys. Chem., 1984, 88, 5768-5769.
$170 \mathrm{~W}$. Sun and G. Ceder, Induction time of a polymorphic transformation, CrystEngComm, 2017, 19, 4576-4585.

171 D. Bonn and N. Shahidzadeh, Multistep crystallization processes: How not to make perfect single crystals, Proc. Natl. Acad. Sci. U. S. A., 2016, 113, 13551-13553.

172 D.-K. Bučar, R. W. Lancaster and J. Bernstein, Disappearing polymorphs revisited, Angew. Chem., Int. Ed., 2015, 54, 6972-6993.

173 Y. Sun, et al., Low-temperature solution synthesis of few-layer $1 \mathrm{~T}^{\prime}$-MoTe2 nanostructures exhibiting lattice compression, Angew. Chem., Int. Ed., 2016, 55, 2830-2834.

174 Y. Sun, K. Fujisawa, M. Terrones and R. E. Schaak, Solution synthesis of few-layer WTe2 and $\mathrm{Mo}_{(x)} \mathrm{W}_{(1-x)} \mathrm{Te}_{2}$ nanostructures, J. Mater. Chem. C, 2017, 5, 11317-11323.

175 N. Savjani, et al., Synthesis of lateral size-controlled monolayer1H-MoS2@oleylamine as supercapacitor electrodes, Chem. Mater., 2016, 28, 657-664.

176 D. Son, et al., Colloidal synthesis of uniform-sized molybdenum disulfide nanosheets for wafer-scale flexible nonvolatile memory, Adv. Mater., 2016, 28, 9326-9332.

177 W. Jung, et al., Colloidal synthesis of single-layer MSe2 $(\mathrm{M}=\mathrm{Mo}, \mathrm{W})$ nanosheets via anisotropic solution-phase growth approach, J. Am. Chem. Soc., 2015, 137, 7266-7269.

178 L. Cheng, et al., Ultrathin WS2 nanoflakes as a highperformance electrocatalyst for the hydrogen evolution reaction, Angew. Chem., Int. Ed., 2014, 53, 7860-7863.

179 D. Sun, S. Feng, M. Terrones and R. E. Schaak, Formation and interlayer decoupling of colloidal MoSe2 nanoflowers, Chem. Mater., 2015, 27, 3167-3175.

180 Y. Sun, et al., Low-temperature solution synthesis of transition metal dichalcogenide alloys with tunable optical properties, J. Am. Chem. Soc., 2017, 139, 11096-11105.

$181 \mathrm{X}$. Zhou, et al., Fast colloidal synthesis of scalable Morich hierarchical ultrathin $\operatorname{MoSe}_{(2-x)}$ nanosheets for highperformance hydrogen evolution, Nanoscale, 2014, 6, 11046-11051.

182 C. Zhang, et al., Phosphine-free synthesis and shape evolution of MoSe2 nanoflowers for electrocatalytic hydrogen evolution reactions, CrystEngComm, 2018, 20, 2491-2498.

183 O. E. Meiron, V. Kuraganti, I. Hod, R. Bar-Ziv and M. BarSadan, Improved catalytic activity of $\mathrm{Mo}_{(1-x)} \mathrm{W}_{(x)} \mathrm{Se}_{2}$ alloy nanoflowers promotes efficient hydrogen evolution reaction in both acidic and alkaline aqueous solutions, Nanoscale, 2017, 9, 13998-14005.

184 R. Mastria, et al., In-plane aligned colloidal 2D WS2 nanoflakes for solution-processable thin films with high planar conductivity, Sci. Rep., 2019, 9, 9002.

185 D. D. Vaughn, R. J. Patel, M. A. Hickner and R. E. Schaak, Single-Crystal Colloidal Nanosheets of GeS and GeSe, J. Am. Chem. Soc., 2010, 132, 15170-15172.

186 M. M. Kobylinski, C. Ruhmlieb, A. Kornowski and A. Mews, Hexagonally shaped two-dimensional tin(II) sulfide nanosheets: Growth model and controlled structure formation, J. Phys. Chem. C, 2018, 122, 5784-5795.

187 Z. Deng, et al., Solution synthesis of ultrathin singlecrystalline SnS nanoribbons for photodetectors via phase 
transition and surface processing, ACS Nano, 2012, 6, 6197-6207.

188 J. Q. Geisenhoff, A. K. Tamura and A. M. Schimpf, Using ligands to control reactivity, size and phase in the colloidal synthesis of WSe2 nanocrystals, Chem. Commun., 2019, 55, 8856-8859.

189 D. D. Vaughn, S. In Il and R. E. Schaak, A precursor-limited nanoparticle coalescence pathway for tuning the thickness of laterally-uniform colloidal nanosheets: The case of SnSe, ACS Nano, 2011, 5, 8852-8860.

190 K. H. Park, K. Jang, S. Kim, H. J. Kim and S. U. Son, Phasecontrolled one-dimensional shape evolution of InSe nanocrystals, J. Am. Chem. Soc., 2006, 128, 14780-14781.

191 D. Yoo, M. Kim, S. Jeong, J. Han and J. Cheon, Chemical synthetic strategy for single-layer transition-metal chalcogenides, J. Am. Chem. Soc., 2014, 136, 14670-14673.

192 S. Jeong, D. Yoo, J. Jang, M. Kim and J. Cheon, Welldefined colloidal 2-D layered transition-metal chalcogenide nanocrystals via generalized synthetic protocols, J. Am. Chem. Soc., 2012, 134, 18233-18236.

193 W. Li, et al., Flowerlike WSe2 and WS2 microspheres - onepot synthesis, formation mechanism and application in heavy metal ion sequestration, Chem. Commun., 2016, 52, 4481-4484.

194 Y. Xie, et al., Metallic-like stoichiometric copper sulfide nanocrystals: Phase- and shape-selective synthesis, nearinfrared surface plasmon resonance properties, and their modeling, ACS Nano, 2013, 7, 7352-7369.

195 J. Song, et al., Solvothermal growth of bismuth chalcogenide nanoplatelets by the oriented attachment mechanism an in situ PXRD study, Chem. Mater., 2015, 27, 3471-3482.

196 C. Schliehe, et al., Ultrathin PbS sheets by two-dimensional oriented attachment, Science, 2010, 329, 550-553.

197 S. Prabakar, C. W. Bumby and R. D. Tilley, Liquid-phase synthesis of flower-like and flake-like titanium disulfide nanostructures, Chem. Mater., 2009, 21, 1725-1730.

198 O. E. Meiron, L. Houben and M. Bar-Sadan, Understanding the formation mechanism and the $3 \mathrm{D}$ structure of $\mathrm{Mo}\left(\mathrm{S}_{x} \mathrm{Se}_{1-x}\right)_{2}$ nanoflower, RSC Adv., 2015, 5, 88108-88114.

199 X. Geng, et al., Pure and stable metallic phase molybdenum disulfide nanosheets for hydrogen evolution reaction, Nat. Commun., 2016, 7, 10672.

$200 \mathrm{~K}$. Leng, et al., Phase restructuring in transition metal dichalcogenides for highly stable energy storage, ACS Nano, 2016, 10, 9208-9215.

201 M. Z. M. Nasir, C. C. Mayorga-Martinez, Z. Sofer and M. Pumera, Two-dimensional 1T-phase transition metal dichalcogenides as nanocarriers to enhance and stabilize enzyme activity for electrochemical pesticide detection, ACS Nano, 2017, 11, 5774-5784.

202 E. Rahmanian, et al., 1T-phase tungsten chalcogenides (WS2, WSe2, WTe2) decorated with TiO2 nanoplatelets with enhanced electron transfer activity for biosensing applications, ACS Appl. Nano Mater., 2018, 1, 7006-7015.

203 W. Wei, K. Sun and Y. H. Hu, An efficient counter electrode material for dye-sensitized solar cells-flower-structured
1T metallic phase MoS2, J. Mater. Chem. A, 2016, 4, 12398-12401.

204 P. Huang, et al., Water-Soluble 2D Transition Metal Dichalcogenides as the Hole-Transport Layer for Highly Efficient and Stable $\mathrm{p}-\mathrm{i}-\mathrm{n}$ Perovskite Solar Cells, ACS Appl. Mater. Interfaces, 2017, 9, 25323-25331.

205 M. Piao, et al., Crystal phase control synthesis of metallic 1T-WS2 nanosheets incorporating single walled carbon nanotubes to construct superior microwave absorber, J. Alloys Compd., 2020, 815, 152335.

206 L. Cai, et al., Vacancy-induced ferromagnetism of MoS2 nanosheets, J. Am. Chem. Soc., 2015, 137, 2622-2627.

207 C. Yang, et al., Phase-driven magneto-electrical characteristics of single-layer MoS2, Nanoscale, 2016, 8, 5627-5633.

208 J. D. Benck, T. R. Hellstern, J. Kibsgaard, P. Chakthranont and T. F. Jaramillo, Catalyzing the hydrogen evolution reaction (HER) with molybdenum sulfide nanomaterials, ACS Catal., 2014, 4, 3957-3971.

209 Z. Gholamvand, et al., Comparison of liquid exfoliated transition metal dichalcogenides reveals MoSe2 to be the most effective hydrogen evolution catalyst, Nanoscale, 2016, 8, 5737-5749.

$210 \mathrm{H}$. Li, et al., Activating and optimizing MoS2 basal planes for hydrogen evolution through the formation of strained sulphur vacancies, Nat. Mater., 2016, 15, 48-53.

211 D. Voiry, et al., The role of electronic coupling between substrate and 2D MoS2 nanosheets in electrocatalytic production of hydrogen, Nat. Mater., 2016, 15, 1003-1009.

212 Y. Yan, et al., Vertically oriented MoS2 and WS2 nanosheets directly grown on carbon cloth as efficient and stable 3-dimensional hydrogen-evolving cathodes, J. Mater. Chem. A, 2015, 3, 131-135.

213 M. Zou, et al., WSe 2 and $\mathrm{W}\left(\mathrm{Se}_{x} \mathrm{~S}_{1-x}\right)_{2}$ nanoflakes grown on carbon nanofibers for the electrocatalytic hydrogen evolution reaction, J. Mater. Chem. A, 2015, 3, 18090-18097.

214 L. Lin, et al., Engineered 2D Transition Metal Dichalcogenides-A Vision of Viable Hydrogen Evolution Reaction Catalysis, Adv. Energy Mater., 2020, 10, 1903870.

215 Q. Lu, Y. Yu, Q. Ma, B. Chen and H. Zhang, 2D TransitionMetal-Dichalcogenide-Nanosheet-Based Composites for Photocatalytic and Electrocatalytic Hydrogen Evolution Reactions, Adv. Mater., 2016, 28, 1917-1933.

216 T. Shinagawa, A. T. Garcia-Esparza and K. Takanabe, Insight on Tafel slopes from a microkinetic analysis of aqueous electrocatalysis for energy conversion, Sci. Rep., 2015, 5, 13801.

217 J. K. Nørskov, et al., Trends in the exchange current for hydrogen evolution, J. Electrochem. Soc., 2005, 152, J23.

218 B. Hinnemann, et al., Biomimetic hydrogen evolution: MoS2 nanoparticles as catalyst for hydrogen evolution, J. Am. Chem. Soc., 2005, 127, 5308-5309.

219 M. V. Bollinger, et al., One-dimensional metallic edge states in MoS2, Phys. Rev. Lett., 2001, 87, 196803.

220 A. Parija, et al., Mapping catalytically relevant edge electronic states of MoS2, ACS Cent. Sci., 2018, 4, 493-503.

221 C. Tsai, K. Chan, F. Abild-Pedersen and J. K. Nørskov, Active edge sites in MoSe2 and WSe2 catalysts for the 
hydrogen evolution reaction: a density functional study, Phys. Chem. Chem. Phys., 2014, 16, 13156-13164.

222 C. Tsai, K. Chan, J. K. Nørskov and F. Abild-Pedersen, Theoretical insights into the hydrogen evolution activity of layered transition metal dichalcogenides, Surf. Sci., 2015, 640, 133-140.

223 M. A. Lukowski, et al., Enhanced hydrogen evolution catalysis from chemically exfoliated metallic MoS2 nanosheets, J. Am. Chem. Soc., 2013, 135, 10274-10277.

224 S. S. Chou, et al., Understanding catalysis in a multiphasic two-dimensional transition metal dichalcogenide, Nat. Commun., 2015, 6, 8311.

225 Y. Zhou, et al., Revealing the contribution of individual factors to hydrogen evolution reaction catalytic activity, Adv. Mater., 2018, 30, 1706076.

226 L. Wang, et al., Self-optimization of the active site of molybdenum disulfide by an irreversible phase transition during photocatalytic hydrogen evolution, Angew. Chem., Int. Ed., 2017, 56, 7610-7614.

$227 \mathrm{~J}$. Zhu, et al., Boundary activated hydrogen evolution reaction on monolayer MoS2, Nat. Commun., 2019, 10, 1348.

228 Y. Ouyang, et al., Activating Inert Basal Planes of MoS2 for Hydrogen Evolution Reaction through the Formation of Different Intrinsic Defects, Chem. Mater., 2016, 28, 4390-4396.

229 Q. Ji, et al., Metallic vanadium disulfide nanosheets as a platform material for multifunctional electrode applications, Nano Lett., 2017, 17, 4908-4916.

230 R. Naz, et al., Highly defective 1T-MoS2 nanosheets on 3D reduced graphene oxide networks for supercapacitors, Carbon, 2019, 152, 697-703.

$231 \mathrm{X}$. Wang, et al., Unveiling highly ambient-stable multilayered 1T-MoS2 towards all-solid-state flexible supercapacitors, J. Mater. Chem. A, 2019, 7, 19152-19160.

232 B. $\mathrm{Xu}$, et al., Adsorption and diffusion of lithium on 1T-MoS2 monolayer, Comput. Mater. Sci., 2014, 93, 86-90.

233 T. Xiang, et al., Vertical 1T-MoS2 nanosheets with expanded interlayer spacing edged on a graphene frame for high rate lithium-ion batteries, Nanoscale, 2017, 9, 6975-6983.

234 X. Cao, C. Tan, X. Zhang, W. Zhao and H. Zhang, SolutionProcessed Two-Dimensional Metal Dichalcogenide-Based Nanomaterials for Energy Storage and Conversion, Adv. Mater., 2016, 28, 6167-6196.

235 S. Shi, Z. Sun and Y. H. Hu, Synthesis, stabilization and applications of 2-dimensional 1T metallic MoS2, J. Mater. Chem. A, 2018, 6, 23932-23977.

236 Q. Yun, et al., Layered Transition Metal DichalcogenideBased Nanomaterials for Electrochemical Energy Storage, Adv. Mater., 2020, 32, 1903826.

237 L. Tao, et al., 1T' transition metal telluride atomic layers for plasmon-free SERS at femtomolar levels, J. Am. Chem. Soc., 2018, 140, 8696-8704.

238 B. Radisavljevic, A. Radenovic, J. Brivio, V. Giacometti and A. Kis, Single-layer MoS2 transistors, Nat. Nanotechnol., 2011, 6, 147-150.
239 Z. Yin, et al., Single-layer MoS2 phototransistors, ACS Nano, 2012, 6, 74-80.

240 O. Lopez-Sanchez, D. Lembke, M. Kayci, A. Radenovic and A. Kis, Ultrasensitive photodetectors based on monolayer MoS2, Nat. Nanotechnol., 2013, 8, 497-501.

241 B. W. H. Baugher, H. O. H. Churchill, Y. Yang and P. Jarillo-Herrero, Optoelectronic devices based on electrically tunable $\mathrm{p}-\mathrm{n}$ diodes in a monolayer dichalcogenide, Nat. Nanotechnol., 2014, 9, 262-267.

242 S. Das, H.-Y. Chen, A. V. Penumatcha and J. Appenzeller, High performance multilayer MoS2 transistors with scandium contacts, Nano Lett., 2013, 13, 100-105.

243 J. H. Sung, et al., Coplanar semiconductor-metal circuitry defined on few-layer MoTe2 via polymorphic heteroepitaxy, Nat. Nanotechnol., 2017, 12, 1064-1070.

244 X. Xu, et al., Scaling-up Atomically Thin Coplanar Semiconductor-Metal Circuitry via Phase Engineered Chemical Assembly, Nano Lett., 2019, 19, 6845-6852.

245 W. Zhang, R. Mazzarello, M. Wuttig and E. Ma, Designing crystallization in phase-change materials for universal memory and neuro-inspired computing, Nat. Rev. Mater., 2019, 4, 150-168.

246 R. H. Friend and A. D. Yoffe, Electronic properties of intercalation complexes of the transition metal dichalcogenides, Adv. Phys., 1987, 36, 1-94.

247 R. B. Somoano, V. Hadek and A. Rembaum, Alkali metal intercalates of molybdenum disulfide, J. Chem. Phys., 1973, 58, 697-701.

248 R. Zhang, et al., Superconductivity in potassium-doped metallic polymorphs of MoS2, Nano Lett., 2016, 16, 629-636.

249 X.-C. Pan, et al., Pressure-driven dome-shaped superconductivity and electronic structural evolution in tungsten ditelluride, Nat. Commun., 2015, 6, 7805.

250 G. Nam, et al., In-plane anisotropic properties of $1 \mathrm{~T}^{\prime}$-MoS2 layers, Adv. Mater., 2019, 31, 1807764.

251 F. Xia, H. Wang and Y. Jia, Rediscovering black phosphorus as an anisotropic layered material for optoelectronics and electronics, Nat. Commun., 2014, 5, 1-6.

252 I. Pletikosić, M. N. Ali, A. V. Fedorov, R. J. Cava and T. Valla, Electronic structure basis for the extraordinary magnetoresistance in WTe2, Phys. Rev. Lett., 2014, 113, 216601.

253 M. N. Ali, et al., Large, non-saturating magnetoresistance in WTe2, Nature, 2014, 514, 205-208.

254 F. C. Chen, et al., Extremely large magnetoresistance in the type-II Weyl semimetal MoTe2, Phys. Rev. B, 2016, 94, 235154.

255 S. Lee, et al., Origin of extremely large magnetoresistance in the candidate type-II Weyl semimetal $\mathrm{MoTe}_{2-x}$, Sci. Rep., 2018, 8, 13937.

256 J. Yang, et al., Elastic and electronic tuning of magnetoresistance in MoTe2, Sci. Adv., 2017, 3, eaao4949.

257 F. M. Pesci, et al., MoS2/WS2 heterojunction for photoelectrochemical water oxidation, ACS Catal., 2017, 7, 4990-4998. 
258 V. Nicolosi, M. Chhowalla, M. G. Kanatzidis, M. S. Strano and J. N. Coleman, Liquid exfoliation of layered materials, Science, 2013, 340, 1226419.

259 D. McManus, et al., Water-based and biocompatible 2D crystal inks for all-inkjet-printed heterostructures, Nat. Nanotechnol., 2017, 12, 343-350.

260 J. Orangi, F. Hamade, V. A. Davis and M. Beidaghi, 3D Printing of additive-free $2 \mathrm{D} \mathrm{Ti}_{3} \mathrm{C}_{2} \mathrm{~T}_{x}$ (MXene) ink for fabrication of micro-supercapacitors with ultra-high energy densities, ACS Nano, 2020, 14, 640-650.

$261 \mathrm{~W}$. Yang, et al., 3D printing of freestanding MXene architectures for current-collector-free supercapacitors, Adv. Mater., 2019, 31, 1902725.
262 Y.-Y. Peng, et al., All-MXene (2D titanium carbide) solidstate microsupercapacitors for on-chip energy storage, Energy Environ. Sci., 2016, 9, 2847-2854.

263 H. K. Ng, et al., Effects of structural phase transition on thermoelectric performance in lithium-intercalated molybdenum disulfide $\left(\mathrm{Li}_{x} \mathrm{MoS}_{2}\right)$, ACS Appl. Mater. Interfaces, 2019, 11, 12184-12189.

264 G. Eda, et al., Photoluminescence from chemically exfoliated MoS2, Nano Lett., 2011, 11, 5111-5116.

265 C. Guo, et al., High-quality single-layer nanosheets of MS2 (M = Mo, Nb, Ta, Ti) directly exfoliated from AMS2 $(\mathrm{A}=\mathrm{Li}, \mathrm{Na}, \mathrm{K})$ crystals, J. Mater. Chem. C, 2017, 5, 5977-5983. 